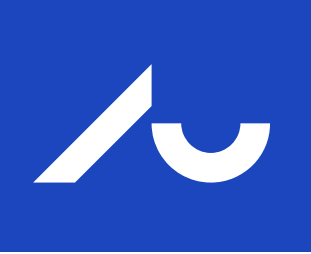

2014-1

David Sloth Pedersen

$\mathrm{PhD}$ Thesis

\title{
A Journey into the Dark Arts of Quantitative Finance
}




\section{A Journey into the Dark Arts of Quantitative Finance}

BY

David Sloth Pedersen

A DOCTORAL THESIS SUBMitTED TO THE

Graduate School of Business

Aarhus UnIVERSITY

in Partial fulfillment of the Ph.D. Degree

SePtember 2013 



\section{Preface}

This thesis is comprised of four self-contained research papers taking us through a journey into the realms of quantitative finance. We join the ranks of practitioners and academic researchers who work on pricing of financial derivatives. A derivative is a financial instrument that derives its value from the value of some underlying quantity or asset, for instance, the stock price of a listed company. This means that a derivative does not have any intrinsic value in itself but should be priced relative to the value of the underlying and other derivatives traded in the market. The importance of sound pricing of such products is stressed by the sheer size of the derivatives market. Looking at just over-the-counter (OTC) products, the market value of outstanding derivatives equaled $\$ 24.7$ trillion by the end of 2012 with a whopping $\$ 632.6$ trillion in notional value (Bank for International Settlements (2013)). The research papers consider different aspects of derivatives pricing; yet, a common aim is to solve problems of a quantitative nature but with a clear financial grounding. Mathematics is an invaluable tool in this endeavor, but the story - we hope - is one of finance.

In the first two chapters we take a step into the risk anatomy of an equity option. Viewed as a package of risk exposures to the underlying stock, the option's fair value must naturally price in all inherent risks and the magnitudes of the risk premiums will depend on the assumed stochastic dynamics of the underlying stock. Nevertheless, this notion is somewhat lost in modern derivatives pricing. The hunger for ever more sophisticated mathematical models to capture the informational content of option prices has called for the use of heavy numerical artillery, even to price plain vanilla options. However, numerical methods act as a black box that fails to reveal the link between the risks that actually drive option prices, the structural properties of the option pricing model, and the 
ultimate effects on the generated surface of option prices. To address this issue, in the first chapter, we expand the true option price around the classical Black-Scholes price. In particular, we show how the true price of an option is determined by a series of premiums on higher-order risks which are not priced under the Black-Scholes model assumptions. In practice, however, options are typically quoted in terms of implied volatilities. So, in the second chapter, we extend our analysis and explore how option risks and the analytical features of the underlying model are translated into implied volatilities.

In the third chapter, we study options on realized variance and, in particular, the impact of jump distributions on the implied volatility of variance. Despite the common notion to include jumps in the variance dynamics, an examination of the distribution of such jumps and its financial implications appear - to the best of our knowledge disturbingly absent from the literature. The chapter shows that the particular distribution of jumps does have a profound impact on the shape of the implied volatility smile of variance. Some jump distributions imply a behavior of the volatility of variance that is clearly at odds with the upward-sloping smile observed in variance markets such as, for example, options on CBOE's VIX index. Evidently, correctly grasping the distribution of jumps in variance is of paramount importance for trading and risk management of volatility derivatives. This chapter attempts to fill this gap.

Finally, in the last chapter we take a step back from the classical pricing paradigm and address risks of the derivatives business which used to be neglected. Prior to the recent financial crisis, dealers tended to ignore the credit risk of high-quality rated counterparties, but the lesson of history has shown that this was a particular dangerous assumption. In addition, as banks became reluctant to lend to each other with the crisis rumbling through the Western economies, the spread between the rate on overnight indexed swaps (OISs) and the LIBOR rate blew up making it apparent that LIBOR is contaminated by credit risk. To keep up with these sudden market changes, dealers today make a number of adjustments when they book derivatives trades, especially, in the OTC market. The credit valuation adjustment (CVA) corrects the price for the expected costs to the dealer due to the possibility that the counterparty may default, while the so-called debit valuation adjustment (DVA) is a correction for the expected benefits to the dealer due to his own default risk. The latter adjustment has the perverse effect that the dealer can book a profit as his default risk increases.

Most controversially, however, dealers often adjust the price for the costs of funding the trade. In the industry, this practice is known as a funding valuation adjustment (FVA). 
When a derivatives desk executes a deal with a client, it backs the trade by hedging it with other dealers in the market. This involves borrowing or lending cash and other assets. Classical derivatives pricing theory rests on the assumption that one can borrow and lend at a unique risk-free rate of interest. This assumption may have been reasonable prior to the crisis with banks funding their hedging strategies at LIBOR. But with drastically increasing spreads, LIBOR is clearly an imperfect proxy of the risk-free rate. In this final chapter we attempt to develop a general pricing framework for valuation of derivatives deals under the presence of complexities such as collateralization, counterparty credit risk and, in particular, funding costs.

\section{Acknowledgments}

First, I would like to thank the assessment committee, Prof. Thomas Kokholm, Dr. Massimo Morini, and Prof. Rolf Poulsen, for useful comments and suggestions. These have definitely improved the thesis.

Secondly, I want to express my sincere gratitude to my thesis advisor Prof. Elisa Nicolato for her unrelenting enthusiasm, continuous support, and immense knowledge. She has taught me almost everything I know about quantitative finance. It has been like a roller-coaster ride. We have been fighting and we have been laughing a lot, and like any roller-coaster ride you want to do it again. I could never have asked for a better advisor and true friend.

Moreover, I would like to thank my co-advisor Prof. Peter Løchte Jørgensen for being the one who encouraged me to pursue a Ph.D. degree in finance and for being a great support during the work.

My sincere thanks also go to Prof. Damiano Brigo for hosting my stay at Imperial College London and for being an invaluable support and inspiration during the visit.

I am greatly thankful to all of my co-authors, Damiano Brigo, Qing Liu, Elisa Nicolato, Andrea Pallavicini, and Camilla Pisani. Without them this thesis would not have been possible. Also, I would like to thank my fellow $\mathrm{PhD}$ students in the $\mathrm{C}$ building for all the fun we had together. I will dearly miss you all. Special thanks go to my office mate, Lene Gilje Justesen, for putting up with me for three years. If not for me, she would probably have finished a long time ago.

Finally, I am deeply indebted to my family for their enormous love and support. Without you, I would never have managed to get this far. I love you so much. 



\section{Summary}

In the following we provide short summaries of each of the four chapters of the thesis.

\section{English summary}

In Chapter 1 we derive a closed-form expansion of option prices in terms of BlackScholes prices and higher-order Greeks. We show how the true price of an option less its Black-Scholes price is given by a series of premiums on higher-order risks that are not priced under the Black-Scholes model assumptions. The expansion can be used for a broad class of option pricing models with dynamics governed by time-changed Brownian motions. Specifically, we study expansions for exponential Lévy models such as the Variance Gamma and the Normal Inverse Gaussian models as well as their stochastic volatility counterparts, e.g., the VGSV and NIGSV models. Moreover, we consider extensions of the expansion to a more general subclass of affine jump-diffusion models for which the pricing transform may not be known in closed form. This chapter is based on a research paper published in Quantitative Finance.

In Chapter 2 we derive a closed-form expansion of implied volatility and at-themoney skew. The expansion is simple, transparent, and easy to implement. It is based on a mixing representation of the underlying model. We apply the expansion to the Heston stochastic volatility model as well as the Variance Gamma model and provide a clear analysis of the at-the-money term structure of implied volatility. However, the expansion may be used for a broad class of option pricing models displaying both jumps and stochastic volatility in the underlying dynamics. Finally, we explore two domains of application of 
the expansion: one as a control variate to speed up Fourier option pricing, and another one as a fast, first-order approach for calibration to at-the-money volatilities and skews.

In Chapter 3 we examine the impact of the distribution of jumps in realized variance. We show that the jump distribution has a profound impact on the characteristics and shape of the implied volatility of variance smile. Some jump distributions predict implied volatilities that are clearly at odds with the upward-sloping volatility skew observed in variance markets. We consider a tractable affine stochastic volatility model that generalizes the seminal Heston (1993) model by augmenting it to jumps in the instantaneous variance. The model allows us to isolate the unique impact of the jump distribution and we provide sufficient conditions for the asymptotic behavior of the volatility of variance for small and large strikes. In particular, we show that for several specific jump distributions, one obtains fundamentally different shapes of the implied volatility of variance smile.

In Chapter 4 we develop an arbitrage-free framework for consistent valuation of OTC derivative trades with collateralization, counterparty credit risk, and funding costs. Based on the risk-neutral pricing principle, we derive a general pricing equation where CVA, DVA, and FVA are introduced by simply modifying the payout cash-flows of the deal. Funding risk breaks the bilateral nature of the deal price and makes the pricing problem a highly non-linear and recursive one. This means that FVA is not generally an additive adjustment as commonly assumed by market participants. Our framework addresses common market practices of ISDA governed deals without restrictive assumptions on collateral margin payments and close-out netting rules. In particular, we allow for asymmetric collateral and funding rates. The pricing equation can be cast as a set of iterative equations that can be solved by least-squares Monte Carlo and we propose such a simulation algorithm. Our numerical results confirm that funding risk does have a non-trivial impact on the deal price.

\section{Dansk resumé}

I kapitel 1 udleder vi en ekpansion af optionspriser som funktion af Black-Scholes priser og højere ordens Greeks. Vi viser, at prisen på en option fratrukket sin Black-Scholes pris er givet ved en kombination af præmier på højere ordensrisici, der ikke er prisfastsat under 
Black-Scholes' modelantagelserne. Ekspansionen kan anvendes på en bred vifte af modeller for optionsprisfastsættelse, hvor den underliggende dynamik er drevet af Brownske bevægelser på stokastisk tid. Specifikt studerer vi ekspansioner for eksponentielle Lévymodeller, som f.eks. Variance Gamma og Normal Inverse Gaussian modellerne, samt de tilsvarende modeller med stokastisk volatilitet, dvs. VGSV og NIGSV modellerne. Ydermere undersøger vi udvidelser af ekspansionen til en mere generel delklasse af affine spring-diffusionsmodeller, hvor Fourier-transformationer til optionsprisfastsættelse ikke findes i lukket form. Dette kapitel er baseret på en forskningsartikel publiceret i Quantitative Finance.

I kapitel 2 udleder vi en ekspansion for den implicitte volatilitet og for skævheden af at-the-money volatiliteten. Ekspansionen er baseret på en mixing-repræsentation af den underliggende model. Den er simpel, intuitiv og let at implementere. Vi anvender ekspansionen på Hestons stokastiske volatilitetsmodel samt Variance Gamma-modellen, og vi giver en grundig analyse af den at-the-money implicitte volatilitetsstruktur over tid. Ekspansionen kan anvendes på en bred vifte af optionsprisfastsættelsesmodeller med både spring og stokastisk volatilitet i den underliggende dynamik. Ydermere undersøger vi to mulige praktiske anvendelser af ekspansionen. For det første foreslår vi, at den kan anvendes som en kontrolvariabel i forbindelse med optionsprisfastsættelse baseret på Fourier transformation. For det andet kan ekspansionen anvedes til hurtig, førsteordenskalibrering af modeller til at-the-money volatiliteter og skævhedsværdier.

I kapitel 3 undersøger vi effekten af sandsynlighedsfordelingen for spring i realiseret varians. Specifikt viser vi, at springfordelingen har en afgørende betydning for formen på det såkaldte implicitte volatilitetssmil for variansoptioner. Nogle springfordelinger leder til implicitte volatiliteter, der er tydeligt inkonsistente med den positive hældning på volatilitetssmilet, som man observerer i variansmarkeder. Vi anvender en affin stokastisk volatilitetsmodel, der generaliserer den populære Heston (1993) model ved at tillade spring i variansen. Modellen gør det muligt at isolere effekten af springfordelingen, og vi udleder tilstrækkelige betingelser for variansvolatilitetens asymptotiske adfærd for små og store optionsstrike-værdier. For en række specifikke fordelinger for spring i variansen viser vi, at disse fører til fundamentalt forskellige volatilitetssmil for variansoptioner.

I kapitel 4 udvikler vi en konsistent værdiansættelsetilgang for OTC handler un- 
der sikkerhedsstillelse, modpartskreditrisiko og finansieringsomkostninger. Vi udleder en generel risikoneutral prisligning, hvor CVA, DVA og FVA indgår som simple modifikationer af handlens payout-pengestrømme. Finansieringsrisiko bryder handelsprisens bilaterale natur og gør prisfastsættelse til et særdeles ikke-lineært og rekursivt problem. Dette betyder, at FVA ikke generelt kan betragtes som en additiv priskorrektion, som det ellers ofte antages blandt praktikere. Vores værdiansættelsestilgang imødekommer markedspraksis for ISDA-regulerede handler uden at opstille restriktive antagelser for sikkerhedsstillelsesproceduren og reglerne for såkaldt close-out netting. Konkret tillader vi asymmetriske renter både på de sikkedsstillede aktiver og i forbindelse med finansieringen af handlens risikoafdækningsstrategi. Vores prisligning kan løses iterativt ved hjælp af least-squares Monte Carlo metoden og vi opstiller en konkret simulationsalgoritme. Vores numeriske resultater bekræfter, at finsieringsrisiko har en ikke-triviel betydning for handelsprisen. 


\section{Contents}

Preface $\quad$ i

Summary $\quad$ v

$\begin{array}{ll}\text { Contents } & \text { ix }\end{array}$

1 Risk Adjustments of Option Prices under Time-changed Dynamics 1

1.1 Introduction . . . . . . . . . . . . . . . . . . . . . 2

1.2 General Model Setup and Derivation of Expansions . . . . . . . . . . . . 5

1.3 Expansions under Lévy Dynamics . . . . . . . . . . . . . . . . . . . . 10

1.4 Expansions under Lévy-Stochastic Volatility Dynamics . . . . . . . . . . . 19

1.5 Expansions under Affine Dynamics . . . . . . . . . . . . . . . . . 26

1.6 Conclusion . . . . . . . . . . . . . . . . . . . . . . . . 33

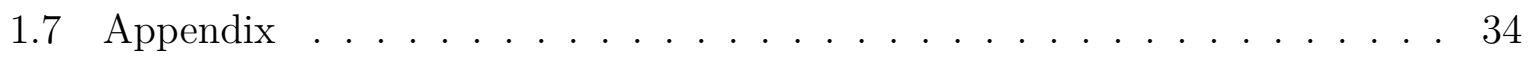

2 Closed-form Expansions of the Volatility Smile in a Mixing Setup 39

2.1 Introduction . . . . . . . . . . . . . . . . . 40

2.2 The Mixing Setup . . . . . . . . . . . . . . . . . . . . . . 43

2.3 Implied Volatility Expansions . . . . . . . . . . . . . . . . . . 46

2.4 Analytics and Numerics for Benchmark Models . . . . . . . . . . . . 51

2.5 Applications . . . . . . . . . . . . . . . . . . . . . 59

2.6 Conclusion . . . . . . . . . . . . . . . . . . 66

3 The Impact of Jump Distributions on the Volatility of Variance $\quad 67$ 
3.1 Introduction . . . . . . . . . . . . . . . . . . . . . 68

3.2 Options on Realized Variance . . . . . . . . . . . . . . . . . 70

3.3 Preliminary Results on Wing Asymptotics . . . . . . . . . . . . . . . . . 72

3.4 Ornstein-Uhlenbeck Specifications . . . . . . . . . . . . . . . 77

3.5 A Menagerie of Positive Distributions . . . . . . . . . . . . . . . . . . 82

3.6 Full SVJ-v Specifications . . . . . . . . . . . . . . . . . 86

3.7 Conclusion . . . . . . . . . . . . . . . . . . . . . . . . 92

3.8 Appendix . . . . . . . . . . . . . . . . . . . . . 93

4 Consistent Valuation of Collateralized OTC Deals under Counterparty Credit and Funding Risk $\quad 95$

4.1 Introduction . . . . . . . . . . . . . . . . . . . 96

4.2 Collateralized Credit and Funding Valuation Adjustments . . . . . . . . . . 100

4.3 General Pricing Equation for OTC Trades . . . . . . . . . . . . . . . . . 110

4.4 Numerical Results . . . . . . . . . . . . . . . . . . . . . . . . 115

4.5 Extensions . . . . . . . . . . . . . . . . . . . . . . . . 123

4.6 Conclusion . . . . . . . . . . . . . . . . . . . . . 124

$\begin{array}{lr}\text { Bibliography } & 127\end{array}$ 


\section{Chapter 1}

Risk Adjustments of Option Prices under

Time-changed Dynamics 


\title{
Risk Adjustments of Option Prices under Time-changed Dynamics
}

\author{
E. Nicolato ${ }^{\dagger}$ and D. Sloth ${ }^{\dagger}$
}

$\dagger$ Department of Economics and Business, Aarhus University, Aarhus

\begin{abstract}
We derive a closed-form expansion of option prices in terms of Black-Scholes prices and higher-order Greeks. We show how the true price of an option less its BlackScholes price is given by a series of premiums on higher-order risks that are not priced under the Black-Scholes model assumptions. The expansion can be used for a broad class of option pricing models with dynamics governed by time-changed Brownian motions. Specifically, we study expansions for exponential Lévy models such as the Variance Gamma and the Normal Inverse Gaussian models as well as their stochastic volatility counterparts, e.g., the VGSV and NIGSV models. Moreover, we consider extensions of the expansion to a more general subclass of affine jump-diffusion models for which the pricing transform may not be known in closed form.
\end{abstract}

\subsection{Introduction}

Getting to grips with the risk anatomy of an option is paramount for understanding its price. Viewed as a package of risk exposures to the underlying, the option's fair value must naturally price in all inherent risks. Moreover, the magnitudes of the risk premiums will depend on the assumed dynamics of the underlying asset. Yet, the notion is somewhat lost in modern derivatives pricing. The hunger for ever more sophisticated models to capture the informational content of option prices has called for the use of heavy numerical artillery, even to price plain vanilla options. For example, in the presence of 
jumps and stochastic volatility in the underlying dynamics, one often resorts to Fourier transform methods (Carr and Madan (1999), Lewis (2001), and Lipton (2002)). Given the characteristic function of the model, these methods are indeed very efficient for option pricing in terms of accuracy. However, Fourier methods act as a black box that fails to reveal the link between the risks that actually drive option prices, the structural properties of the option pricing model, and the ultimate effects on the generated surface of option prices.

In this paper, we take a step into the anatomy of option prices and explore the direct relation between option risks and the option price for a generalized class of option pricing models. Inspired by the results of Drimus (2011), we expand the true option price around the Black-Scholes price. Despite the empirical shortcomings, its broad market acceptance still makes the model of Black and Scholes (1973) the natural reference point. We show how the true price of an option less its Black-Scholes price is given by a series of premiums on higher-order risks which are not priced under the Black-Scholes model assumptions.

Every day traders hedge their option books against exposures to convexity of volatility and sensitivity of the Delta to the level of volatility; in market jargon, so-called Volga and Vanna risks. Our expansion clarifies how these risks are priced across different option pricing models, and we show how the risk adjustments are directly related to the features and parameters of the particular model at hand. Besides simply demystifying option prices of sophisticated models by bringing them down to the risks that actually generate them, this gives the market participant the opportunity to select the model that prices these risks consistent with his or her own expectations.

Approximations of option prices are by no means the dawn of a new day; attempts in this direction have been the focus of research for as long as we have had options. A significant part of the previous literature deals with computing option prices through an approximation of the risk-neutral density. Abadir and Rockinger (2003), Ait-Sahalia (2002) and Egorov et al. (2003) consider such approximations for diffusion processes, while jump diffusions and their multivariate extensions are considered in $\mathrm{Yu}$ (2007) and Filipovic et al. (2011). In addition, saddlepoint approximations of densities are studied in Rogers and Zane (1999), Xiong et al. (2005) and Aït-Sahalia and Yu (2006). Finally, Sartorelli (2010) and Poulsen and Ribeiro (2012) consider Edgeworth expansions of the density to approximate option prices in the Heston (1993) model.

Other popular approximations for options are based on perturbation and asymptotic methods. A classical reference is Hagan et al. (2002) who employed singular perturbation 
techniques to the PDE of the SABR model assuming small volatility. Lewis (2000) derives an asymptotic expansion for small volatility of volatility in a stochastic volatility model. Similar results were obtained by Lee (2001) assuming slow mean-reversion of volatility, Fouque et al. (2000) study the case of fast mean-reverting volatility, while Antonelli and Scarlatti (2009) make an expansion around zero correlation. Medvedev and Scaillet (2007) include jumps and derive an asymptotic expansion in a general jump-diffusion stochastic volatility model. Recently, Alòs et al. (2007) and Benhamou et al. (2009) have derived approximations of options using Malliavin calculus. Common to these methods is the fact that the approximations are restricted to either short or long maturities.

This work, however, is more closely related to the approach in Lewis (2000) and the recent papers of Alòs (2012), Kristensen and Mele (2011) and, in particular, Drimus (2011) who consider direct expansions of option prices around the Black-Scholes price. These studies are limited to diffusion-type models without jumps. Nonetheless, to generate sufficient variability and asymmetry in short-term returns to match implied volatility skews, we need to allow for jumps. We derive a unifying expansion framework for the broad class of option pricing models driven by a time-changed Brownian motion. This allows us to obtain closed-form expansions of option prices valid under both jumps and stochastic volatility in the underlying dynamics. Analytical and numerical results are presented for popular models such as exponential Lévy models (e.g., Madan et al. (1998), Barndorff-Nielsen (1998)), Lévy stochastic volatility models (Carr et al. (2003)) as well as more general affine model constructions.

For the considered model cases, the accuracy of the expansion is well within one volatility point which is usually the bid-ask spread around at-the-money for even liquid equity underlyings. Moreover, we find that the expansion can be used for speedy, firstorder calibration and to provide smart initial values for a more accurate, but often timeconsuming calibration routine. This is particularly attractive for affine models without a closed-form pricing transform, in which case calibration based on Fourier inversion can be a daunting task in terms of computational time.

The paper is organized as follows. In Section 2 we describe the general model setup and derive the expansion of option prices in terms of the Black-Scholes price and higher-order Greeks. In Section 3 we consider the simplest case of expansions under Lévy dynamics. This is further generalized in Section 4 where we consider the case of Lévy stochastic volatility models, which allows for both jumps and stochastic volatility. In Section 5 we investigate extensions to more general affine jump-diffusion stochastic volatility models. 
Finally, Section 6 concludes the paper. The facts on semimartingale characteristics, cumulant processes and affine processes which are relevant for this work are collected in the Appendix.

\subsection{General Model Setup and Derivation of Expansions}

We consider a general market model specified via a bivariate semimartingale process $(X, V)$ which lives on a given filtered probability space $\left(\Omega, \mathcal{F},\left(\mathcal{F}_{t}\right)_{0 \leqslant t \leqslant T^{*}}, \mathbb{Q}\right)$ and takes values in $\mathbb{R} \times \mathbb{R}_{+}$. The positive component $V$ is assumed to be an increasing process with $V_{0}=0$ and cumulant process $K^{V}(\zeta)$ given by

$$
K_{t}^{V}(\zeta)=\int_{0}^{t} \kappa_{s}^{V}(\zeta) d s \quad \text { with } \quad \kappa_{t}^{V}(\zeta)=\zeta b_{t}^{V}+\int_{\mathbb{R}_{+}}\left(e^{\zeta x}-1\right) F_{t}^{V}(d x)
$$

where $b^{V}$ is a positive predictable process while $F^{V}$ is a transition kernel from $\left(\Omega \times \mathbb{R}_{+}, \mathcal{P}\right)$ to $\left(\mathbb{R}_{+}, \mathcal{B}\right)$ with $F^{V}(\{0\})=0$ and $\int_{\mathbb{R}_{+}}(1 \wedge x) F .(d x)<+\infty$. Hence, the process $V$ admits differential characteristic triplet $\left(b^{V}, 0, F^{V}\right)$ relative to the zero truncation function and the Lévy exponent $\kappa^{V}(\zeta)$ is well defined for any $\zeta \in \mathbb{C}_{-}$where $\mathbb{C}_{-}=\{z \in \mathbb{C}: \Re z \leq 0\}$.

We furthermore assume that the process $V$ is regular enough to satisfy the following property.

Assumption 1. We assume that for all $\zeta \in \mathbb{R}_{-}$the exponentially compensated process $M(\zeta)$ given by

$$
M_{t}(\zeta)=e^{-K_{t}^{V}(\zeta)+\zeta V_{t}}
$$

is a true $\mathbb{Q}$-martingale.

The real-valued component $X$ represents the source of randomness driving the underlying asset price process and it is constructed via the time-change technique. Specifically, we assume that $X$ admits the following representation

$$
X_{t}=\theta V_{t}+W_{V_{t}}
$$

where $W$ is a standard Brownian motion independent of $V$ and the parameter $\theta$ is a real constant introducing skewness in the distributions of $X$ as well as correlation between $X$ and $V$. Thus, $X$ is obtained by Bochner's subordination of a Brownian motion with drift 
to $V$ which can be interpreted either as the stochastic clock or the cumulative random variance of the return-driving process. In this work the second interpretation is more natural and we will refer to $V$ as the total variance process.

For notational convenience, we will often use the re-parameterization $c=\theta+\frac{1}{2}$ and refer to $c$ as the skewness parameter. We shall impose the simplifying constraint that the skewness parameter satisfies $c \leqslant 0$. This assumption is not restrictive in practice but consistent with the negative skew of volatility surfaces observed in equities markets.

Next, we derive the differential characteristics of the process $X$.

Proposition 1. The process $X$ has cumulant process $K^{X}(\zeta)$ given by

$$
K_{t}^{X}(\zeta)=K_{t}^{V}\left(\theta \zeta+\frac{1}{2} \zeta^{2}\right)
$$

and it is well defined for those $\zeta \in \mathbb{C}$ with $\Re \zeta \in[0,-2 \theta]$. Relative to a fixed truncation function $h(x), X$ admits differential triplet $\left(b^{X}, c^{X}, F^{X}\right)$ given by

$$
\begin{aligned}
b_{t}^{X} & =\theta b_{t}^{V}+\int_{\mathbb{R}_{+}} F_{t}^{V}(d u) \int h(x) \mu(d x ; \theta u, u), \\
c_{t}^{X} & =b_{t}^{V}, \\
F_{t}^{X}(B) & =\int_{\mathbb{R}_{+}} \mu(B ; \theta u, u) F_{t}^{V}(d u), \quad B \in \mathcal{B}(\mathbb{R}),
\end{aligned}
$$

where $\mu(d x ; m, v)$ denotes the distribution of a Gaussian variate with mean $m$ and variance $v$.

Proof. Proceeding by conditional expectations, it follows immediately that the process $e^{-K_{t}^{X}(\zeta)+\zeta X_{t}}$ is a local martingale for all those $\zeta \in \mathbb{C}$ for which $K^{X}(\zeta)$ is well-defined. Moreover, we have that

$$
\begin{aligned}
\int_{\mathbb{R}}\left(1 \wedge x^{2}\right) F_{t}^{X}(d x) & =\int_{\mathbb{R}_{+}} \int_{\mathbb{R}}\left(1 \wedge x^{2}\right) \mu(d x ; \theta u, u) F_{t}^{V}(d u) \\
& \leq \int_{\mathbb{R}_{+}}\left(\int_{\mathbb{R}} \mu(d x ; \theta u, u) \wedge \int_{\mathbb{R}} x^{2} \mu(d x ; \theta u, u)\right) F_{t}^{V}(d u) \\
& =\int_{\mathbb{R}_{+}}\left(1 \wedge\left(u+\theta^{2} u^{2}\right)\right) F_{t}^{V}(d u)<\infty,
\end{aligned}
$$

and therefore expression in (1.4) yields a well defined transition kernel $F^{X}$. Finally the fact that $\left(b^{X}, c^{X}, F^{X}\right)$ is the differential triplet of $X$ follows from the uniqueness of the cumulant process and verifying that $K^{X}(\zeta)$ satisfies the following

$$
K_{t}^{X}(\zeta)=\int_{0}^{t}\left(\zeta b_{s}^{X}+\frac{1}{2} \zeta^{2} c_{s}^{X}+\int_{\mathbb{R}}\left(e^{\zeta x}-1-h(x) \zeta\right) F_{s}^{X}(d x)\right) d s .
$$


Finally, assuming a simplified economy with zero interest rates and dividend yields, we specify the dynamics of an asset price process $S$ as follows

$$
S_{t}=S_{0} e^{-K_{t}^{X}(1)+X_{t}}=S_{0} e^{-K_{t}^{V}(c)+X_{t}},
$$

where $K^{V}(c)$ is well-defined as $c \leq 0$. Due to Assumption 1 and the independence between $W$ and $V$, the process $S$ in (1.5) is a true martingale and therefore describes the riskneutral dynamics of the asset price. The generalization to the case of non-zero constant interest rates and dividend yields is straightforward.

The applicability of the presented model class relies on the tractability of the joint Laplace transform of the total variance $V_{T}$ and the cumulant $K_{T}^{V}$ evaluated at $c$

$$
\mathcal{L}_{T}(u, v)=E\left[e^{u K_{T}^{V}(c)+v V_{T}}\right]
$$

defined in the domain

$$
\mathcal{D}_{T}=\left\{(u, v) \in \mathbb{C}^{2}: E\left[\left|e^{u K_{T}^{V}(c)+v V_{T}}\right|\right]<+\infty\right\} .
$$

For example, in order to compute option prices based on Fourier or other transform methods, it is quite crucial that $\mathcal{L}_{T}$ is available in closed or semi-closed form as the characteristic function of the $\log$-price $\log S_{T}$ is given by

$$
E\left[e^{i z \log S_{T}}\right]=S_{0}^{i z} \mathcal{L}_{T}\left(-i z, i z \theta-\frac{1}{2} z^{2}\right)
$$

As we shall see, the Laplace transform $\mathcal{L}_{T}$ is also the computational engine for the option price approximation that we develop.

At the core of our approach is the fact that the price process $S$ in (1.5) can be represented as the product of two positive martingales as follows

$$
S_{t}=S_{0} \xi_{t} e^{-\frac{1}{2} V_{t}+W_{V_{t}}}
$$

where the spot-correcting process $\xi$ is the martingale with mean-value one given by

$$
\xi_{t}=e^{-K_{t}^{V}(c)+c V_{t}}
$$

Notice that if the total variance process $V$ is absolutely continuous, the spot correcting martingale degenerates to the process identically equal to one due to the fact that

$$
V_{t}=\int_{0}^{t} b_{s-}^{V} d s \quad \text { and } \quad K_{t}^{V}(\zeta)=\zeta V_{t}
$$


Hence, to allow for a flexible behavior of the risk-neutral skewness we will implicitly assume the presence of jumps in the paths of the total variance process.

In complete analogy to Romano and Touzi (1997), we can exploit representation (1.8) to express the price of a European call as the risk-neutral expectation of the Black-Scholes price evaluated in the spot-corrected price of the underlying $S_{0} \xi$ and the total variance $V$. Specifically, for a European call option with strike $K$ and expiry $T$, we obtain the following mixing representation for its price $C\left(S_{0}, T\right)$ at time $t=0$

$$
C\left(S_{0}, T\right)=E\left[\left(S_{T}-K\right)^{+}\right]=E\left[C_{B S}\left(S_{0} \xi_{T}, V_{T}\right)\right]
$$

where $C_{B S}(S, V)$ denotes the Black-Scholes price of a call option in terms of the spot price and total variance of the underlying asset. That is,

$$
C_{B S}(S, V)=S \Phi\left(d_{+}\right)-K \Phi\left(d_{-}\right)
$$

where

$$
d_{ \pm}=\frac{x}{\sqrt{V}} \pm \frac{\sqrt{V}}{2},
$$

with $x=\log (S / K)$ denoting the log-moneyness. When the spot-correcting martingale $\xi \equiv 1$, i.e., when $V$ is absolutely continuous or when the skewness parameter $c=0$, we can proceed along the lines of Proposition 3.1 in Renault and Touzi (1996) to show that the generated implied volatility smile is symmetric around the at-the-money point $x=0$. On the other hand, allowing for the presence of jumps in $V$ and letting $c<0$ enables the model to capture the empirically-observed negative volatility skew.

We are now ready to derive a simple, closed-form expansion of the call price in terms of a Black-Scholes price and higher-order Greeks. Like Drimus (2011), we apply a twodimensional Taylor series to expand (1.10) in the underlying asset price and total variance around the point $\left(S_{0}, E V_{T}\right)$. Truncating the expansion to the $n$-th order and recalling that $E\left[\xi_{T}\right]=1$, we obtain the following approximation of option prices at time $t=0$

$$
\begin{aligned}
C\left(S_{0}, T\right) \approx & C_{B S}\left(S_{0}, E V_{T}\right) \\
& +\left.\sum_{k=2}^{n} \sum_{l=0}^{k} \frac{1}{l !(k-l) !} \frac{\partial^{k} C_{B S}}{\partial S^{l} \partial V^{k-l}}\right|_{\left(S_{0}, E V_{T}\right)} S_{0}^{l} E\left[\left(\xi_{T}-1\right)^{l}\left(V_{T}-E V_{T}\right)^{k-l}\right] .
\end{aligned}
$$

Clearly, the $n$-th order approximation given in (1.13) is meaningful only under the assumption that all the moments entering the expression are finite. Also, the practical applicability of the approximation relies on the possibility of computing expectations of 
the type $E\left[V_{T}^{m} \xi_{T}^{n}\right]$, preferably in closed form. Well-known results from probability theory relate these issues to the regularity and the tractability of the joint Laplace transform $\mathcal{L}_{T}$ introduced in (1.6). Sufficient mechanics for computing the moments are summarized in the proposition below.

Proposition 2. Assume that for a given $n \geq 0$ both the points $(0,0)$ and $(-n, n c)$ lie in the interior domain $\stackrel{\circ}{\mathcal{D}}_{T}$ of the Laplace transform $\mathcal{L}_{T}$. Then $E\left[V_{T}^{m} \xi_{T}^{k}\right]<+\infty$ for all $m \geq 0,0 \leq k \leq n$ and it is given by

$$
E\left[V_{T}^{m} \xi_{T}^{k}\right]=\left.\frac{\partial^{m}}{\partial v^{m}} \mathcal{L}(u, v)\right|_{u=-k, v=k c}
$$

Proof. The result follows from the fact that the Laplace transform is analytic in the interior of its domain and can be found in, e.g., Hoffmann-Jørgensen (1994), Section 4.19.

Notice that even when all the moments are finite, there is no guarantee that the expansion (1.13) converges with higher-order approximations capturing the true option prices more accurately. As observed by Drimus (2011) (see also Carr (2000)), this is due to the fact that a Taylor expansion of the Black-Scholes price in the spot and variance has a finite radius of convergence, while here we expand the mixing representation (1.10) over the entire domain $(S, V) \in(0, \infty) \times(0, \infty)$. However, this is not a shortcoming peculiar to our specific approach but rather a common trait of many expansions proposed in the literature.

The major advantage of the expansion (1.13) is in adding transparency to how option prices are generated as deviations from the simplifying Black-Scholes prices through a series of risk-adjustment terms. For example, let us consider the second-order approximation which will play the main role in the numerical examples below. It is given by

$$
\begin{aligned}
C\left(S_{0}, T\right) \approx & C_{B S}+\frac{1}{2} \frac{\partial^{2} C_{B S}}{\partial S^{2}} S_{0}^{2} E\left[\left(\xi_{T}-1\right)^{2}\right] \\
& +\frac{1}{2} \frac{\partial^{2} C_{B S}}{\partial V^{2}} E\left[\left(V_{T}-E V_{T}\right)^{2}\right] \\
& +\frac{\partial^{2} C_{B S}}{\partial S \partial V} S_{0} E\left[\left(\xi_{T}-1\right)\left(V_{T}-E V_{T}\right)\right]
\end{aligned}
$$


where we have dropped the common argument $\left(S_{0}, E V_{T}\right)$. The Greeks are given by

$$
\begin{aligned}
\text { Gamma } & =\frac{\partial^{2} C_{B S}}{\partial S^{2}}(S, V)=\frac{\varphi\left(d_{+}\right)}{S \sqrt{V}} \\
\text { Volga } & =\frac{\partial^{2} C_{B S}}{\partial V^{2}}(S, V)=\frac{S \varphi\left(d_{+}\right)}{4 V^{3 / 2}\left(d_{+} d_{-}-1\right)} \\
\text { Vanna } & =\frac{\partial^{2} C_{B S}}{\partial S \partial V}(S, V)=-\frac{\varphi\left(d_{+}\right) d_{-}}{2 V}
\end{aligned}
$$

with $\varphi(\cdot)$ being the density of the standard normal distribution and $d_{ \pm}$defined as above.

Market participants try to cope with smile effects by hedging their exposures not only to classical Greeks but also to higher-order sensitivities like the Vanna and Volga. This notion is elegantly captured by the expansion. Taking the Black-Scholes price as its reference, the expansion adjusts the option price for volatility convexity and crossconvexity; smile risks that are not priced in the classical Black-Scholes model.

\subsection{Expansions under Lévy Dynamics}

In this section we specialize the general framework described in Section 1.2 to the case where the total variance process $V$ is specified as a Lévy subordinator, i.e.,

$$
V:=\left(Z_{t}\right)_{t \geq 0}
$$

where $Z$ is an increasing process with independent and stationary increments ${ }^{1}$.

For such a process the differential triplet, termed the Lévy-Khintchine triplet, is deterministic and time-independent with

$$
b_{t}^{V}=\beta^{Z} \quad \text { and } \quad F_{t}^{V}(d x)=\nu^{Z}(d x) \text { for all } t \geq 0,
$$

where $\beta^{Z} \geq 0$ and $\nu^{Z}(d x)$, termed the Lévy measure, is a measure on $\mathbb{R}_{+}$such that $\int_{\mathbb{R}_{+}}(1 \wedge x) \nu^{Z}(d x)<\infty$. Thus, also the cumulant process is deterministic and takes the form $K_{t}^{V}(\zeta)=t \cdot \kappa^{Z}(\zeta)$ where $\kappa^{Z}$ is the Lévy-Khintchine exponent

$$
\kappa^{Z}(\zeta)=\zeta \beta^{Z}+\int_{\mathbb{R}_{+}}\left(e^{\zeta x}-1\right) \nu^{Z}(d x)
$$

\footnotetext{
${ }^{1}$ For a comprehensive account on Lévy processes and their applications in financial modeling, we refer the reader to Cont and Tankov (2004)
} 
Assumption 1 is automatically satisfied by any subordinator $Z$, and Proposition 1 implies that the associated $X$ process is a Lévy process

$$
X:=\left(L_{t}\right)_{t \geq 0}
$$

with triplet $\left(\beta^{L}, \gamma^{L}, \nu^{L}\right)$ computed according to equations (1.4)

$$
\begin{aligned}
\beta^{L} & =\theta \beta^{Z}+\int_{\mathbb{R}_{+}} \nu^{Z}(d u) \int h(x) \mu(d x ; \theta u, u), \\
\gamma^{L} & =\beta^{Z}, \\
\nu^{L}(B) & =\int_{\mathbb{R}_{+}} \mu(B ; \theta u, u) \nu^{Z}(d u), \quad B \in \mathcal{B}(\mathbb{R}) .
\end{aligned}
$$

Correspondingly, the resulting asset-pricing model in (1.5) belongs to the class of exponential Lévy models.

If we furthermore assume that there exists $\varepsilon>0$ such that the Lévy exponent $\kappa^{Z}$ in (1.16) satisfies $\kappa^{Z}(\varepsilon)<\infty$, then the expansion (1.13) can be truncated at any desired order as Proposition 2 ensures that $E\left[V_{T}^{m} \xi_{T}^{n}\right]<\infty$ for all $n, m \geq 0$, with formula (1.14) yielding

$$
E\left[V_{T}^{m} \xi_{T}^{n}\right]=\left.e^{-n \kappa^{Z}(c) T} \frac{d^{m}}{d v^{m}} e^{T \kappa^{Z}(v)}\right|_{v=n c} .
$$

We note in passing that we may also employ the following alternative representation

$$
E\left[V_{T}^{m} \xi_{T}^{n}\right]=e^{\left(\kappa^{Z}(n c)-n \kappa^{Z}(c)\right) T} E^{\mathbb{Q}^{n}}\left[V_{T}^{m}\right],
$$

where $\mathbb{Q}^{n}$ is the probability measure defined by the following Esscher transform

$$
\left.\frac{d \mathbb{Q}^{n}}{d \mathbb{Q}}\right|_{\mathcal{F}_{t}}=e^{-\kappa^{Z}(n c) t+n c V_{t}}
$$

Under $\mathbb{Q}^{n}$ the total variance process $V$ is a subordinator $\widetilde{Z}$ with exponentially tilted Lévy measure

$$
\widetilde{\nu}(d u)=e^{n c u} \nu^{Z}(d u)
$$

and unchanged drift $\widetilde{\beta}=\beta^{Z}$.

\subsubsection{Specific exponential Lévy models}

Popular exponential Lévy models that can be obtained in this framework include the Hyperbolic models suggested by Eberlein and Keller (1995), the Normal Inverse Gaussian 
(NIG) process proposed in Barndorff-Nielsen (1998), the Variance Gamma (VG) process introduced by Madan and Seneta (1990), the Meixner process studied in Schoutens (2002) and the CGMY process considered in Carr et al. (2002) and Boyarchenko and Levendorskii (2002).

In the following we concentrate on the VG model and the NIG model. While very easy to describe, the two specifications differ considerably in their structural properties, with the VG process displaying paths of finite variation and the NIG process displaying paths of infinite variation.

The VG model is obtained by specifying the total variance $V$ process as a Gammasubordinator, i.e., by setting the unit-time distribution as a Gamma law with density function

$$
f(x)=\frac{b^{a}}{\Gamma(a)} x^{a-1} e^{-b x}, \quad x>0, \quad a, b>0 .
$$

The corresponding Lévy exponent is given by

$$
\kappa^{Z}(\zeta)=\log \left(\frac{1}{\left(1-\zeta b^{-1}\right)^{a}}\right)=\int_{0}^{\infty}\left(e^{\zeta x}-1\right) \frac{a e^{-b x}}{x} d x,
$$

implying that the drift $\beta^{Z}=0$, while the Lévy measure $\nu^{Z}(d x)=\frac{a e^{-b x}}{x} d x$. The mean and variance are given by

$$
\frac{d}{d \zeta} \kappa^{Z}(0)=\frac{a}{b}, \quad \frac{d^{2}}{d \zeta^{2}} \kappa^{Z}(0)=\frac{a}{b^{2}} .
$$

Notice that in the VG model the expansion of option prices can be truncated at any order $n$ as the Lévy exponent $\kappa^{Z}$ in (1.22) is finite for $\zeta<b$. Moreover, the exponential tilting (1.21) preserves the Gamma property implying that the total variance process $V$ is still a Gamma-subordinator under $\mathbb{Q}^{n}$ defined in (1.20), albeit with different parameters

$$
\widetilde{a}=a, \quad \widetilde{b}=b-n c .
$$

Therefore, the representation (1.19) readily provides all the cross-moments $E\left[V_{T}^{m} \xi_{T}^{n}\right]$ via a re-parametrization of simple moments of a Gamma distribution. The explicit expressions entering the second-order approximation (1.15) are given as follows

$$
\begin{array}{cc}
E\left[V_{T}\right]=T \frac{a}{b}, \quad E\left[V_{T}^{2}\right]=T^{2} \frac{a^{2}}{b^{2}}+T \frac{a}{b^{2}}, \\
E\left[V_{T} \xi_{T}\right]=E^{\mathbb{Q}^{1}}\left[V_{T}\right]=T \frac{a}{b-c}, & E\left[\xi_{T}^{2}\right]=\left(\frac{\left(1-c b^{-1}\right)^{2}}{1-2 c b^{-1}}\right)^{a T} .
\end{array}
$$


While very convenient for nesting the VG model in the general framework of Section 1.2, the above description in terms of the parameters $(a, b, c)$ is not the most commonly used in the literature. Madan et al. (1998), to whom we refer below, adopt another parametrization $(\sigma, \nu, \theta)$ which stems from the equivalent time-change representation

$$
X_{t}=\theta V_{t}+\sigma W_{V_{t}}
$$

where the unit-time is specified as a Gamma law with mean one and variance $\nu$. The relationship between the two parameterizations is given by

$$
\nu=\frac{1}{a}, \quad \sigma^{2}=\frac{a}{b}, \quad \theta=\left(c-\frac{1}{2}\right) \frac{a}{b} .
$$

For later use, we also report the parametrization $(C, G, M)$ employed in Schoutens et al. (2004) which is based on the alternative representation of a VG process $X$ as a difference of two independent Gamma-subordinators

$$
X_{t}=\Gamma_{t}(C, M)-\Gamma_{t}(C, G)
$$

where

$$
\begin{aligned}
C & =a \\
M & =\left(\sqrt{\left(\frac{c-1 / 2}{2 b}\right)^{2}+\frac{1}{2 b}}+\frac{c-1 / 2}{2 b}\right)^{-1} \\
G & =\left(\sqrt{\left(\frac{c-1 / 2}{2 b}\right)^{2}+\frac{1}{2 b}}-\frac{c-1 / 2}{2 b}\right)^{-1}
\end{aligned}
$$

As a second example, we consider the NIG model. This model is obtained by defining $V$ as a subordinator with unit-time distributed according to an Inverse Gaussian (IG) law with density function

$$
f(x)=\frac{a}{\sqrt{2 \pi}} e^{a b} x^{-\frac{3}{2}} e^{-\left(b^{2} x+\frac{a^{2}}{x}\right) / 2}, \quad x>0, \quad a, b>0 .
$$

The Lévy exponent is given by

$$
\kappa(\zeta)=a b-a \sqrt{b^{2}-2 \zeta}=\int_{0}^{\infty}\left(e^{\zeta x}-1\right) \frac{a}{\sqrt{2 \pi}} \frac{e^{-\frac{1}{2} b^{2} x}}{x^{\frac{3}{2}}} d x
$$


and the mean and variance read as follows

$$
\frac{d}{d \zeta} \kappa^{Z}(0)=\frac{a}{b} \quad \frac{d^{2}}{d \zeta^{2}} \kappa^{Z}(0)=\frac{a}{b^{3}}
$$

Similar to the VG case, the closed-form expansion of NIG option prices can be truncated at any order $n$ as $\kappa^{Z}(\zeta)<\infty$ for $\zeta<b^{2} / 2$. From the expression of the Lévy measure in (1.24) we see that the IG distribution is closed under the exponential tilting in (1.21). Under the measure $\mathbb{Q}^{n}$ in $(1.20)$, the total variance process $V$ is still an IG-subordinator with parameters

$$
\widetilde{a}=a, \quad \widetilde{b}=\sqrt{b^{2}-2 n c} .
$$

Once again it is straightforward to derive the cross-moments from moments of the IG distribution via representation (1.19). The moments entering the second-order approximation are given by

$$
\begin{aligned}
E\left[V_{T}\right]=T \frac{a}{b}, & E\left[V_{T}^{2}\right]=T^{2} \frac{a^{2}}{b^{2}}+T \frac{a}{b^{3}}, \\
E\left[V_{T} \xi_{T}\right]=T \frac{a}{\sqrt{b^{2}-2 c}}, & E\left[\xi_{T}^{2}\right]=e^{a T\left(2 \sqrt{b^{2}-2 c}-\sqrt{b^{2}-4 c}-b\right)} .
\end{aligned}
$$

Also the NIG process is usually not described in terms of the parameters $(a, b, c)$. In what follows we will refer to the $(\alpha, \beta, \delta)$-parameterization employed for example in Këllezi and Webber (2004) which corresponds to the following time-change construction

$$
X_{t}=\beta \delta^{2} V_{t}+\delta W_{V_{t}}
$$

where the unit-time is specified as an IG law with parameters $a=1$ and $b=\delta \sqrt{\alpha^{2}-\beta^{2}}$. The relationship between the two parameterizations is given by

$$
a=\delta, \quad b=\sqrt{\alpha^{2}-\beta^{2}}, \quad c=\beta+\frac{1}{2} .
$$

\subsubsection{Numerical results}

Let us bring the approximation to the numbers. We do this in two steps: First, we look at the numerical accuracy of the approximation to determine its domain of application. The implied volatilities of the expansion truncated to 2nd order are compared to the true ones under both VG dynamics and NIG dynamics.

Figure 1.1 compares the true implied volatility surface with the volatility surface implied by the approximation. For the VG model we use the original parameters $\sigma=$ 


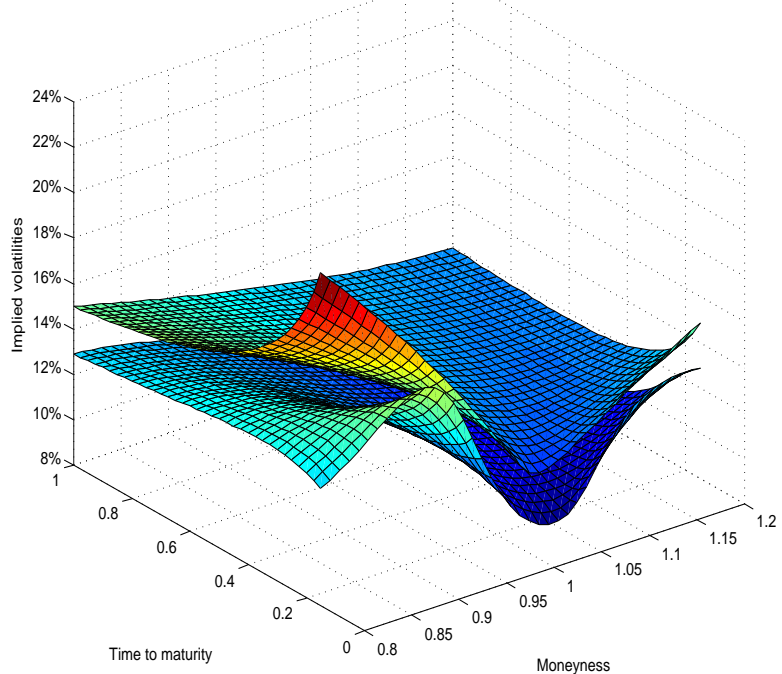

(a) VG dynamics

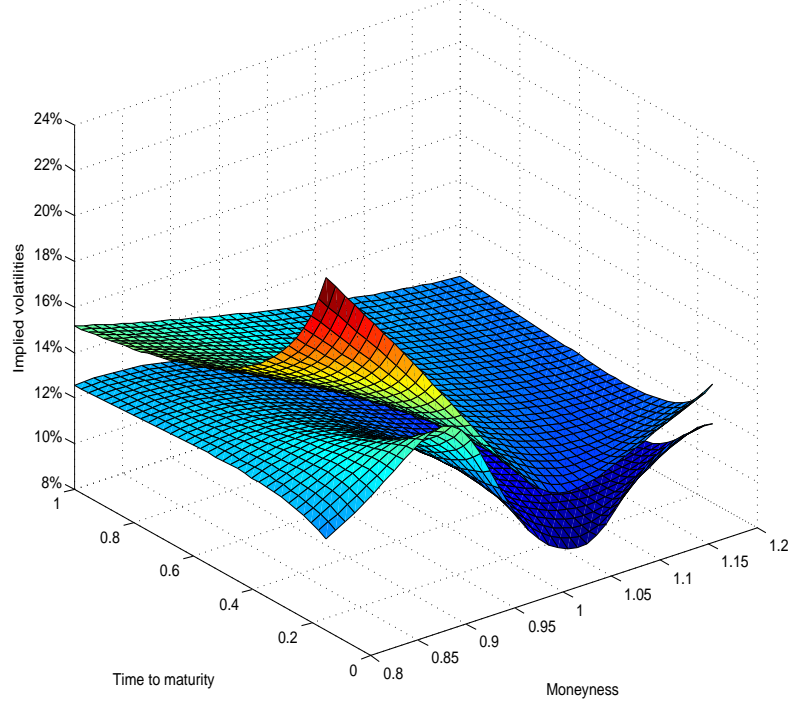

(b) NIG dynamics

Figure 1.1: Approximated volatilities (lower surface) against true volatilities (upper surface). The approximate surface is moved down by $200 \mathrm{bps}$ for ease of interpretation.

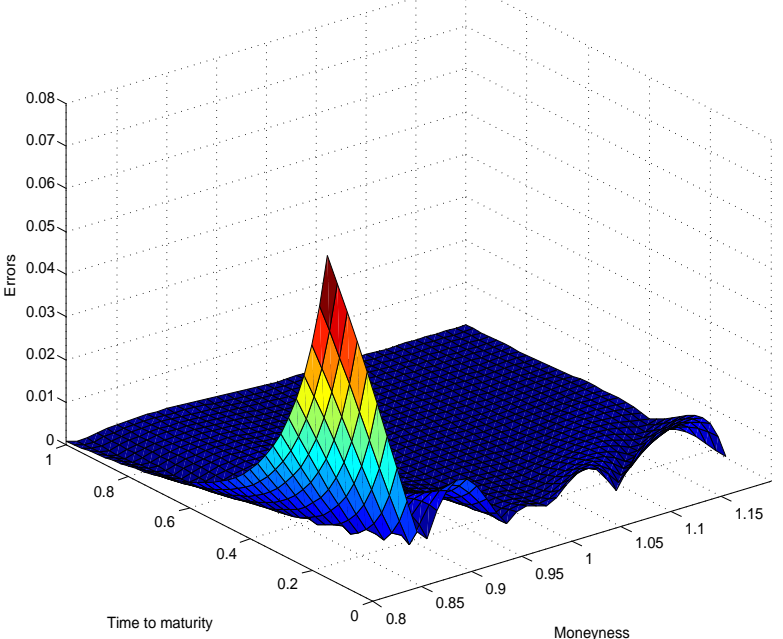

(a) VG dynamics

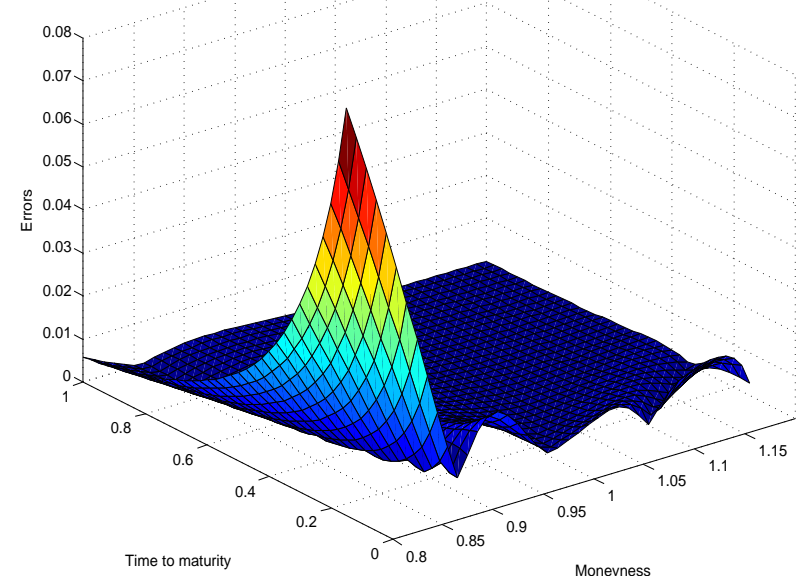

(b) NIG dynamics

Figure 1.2: Absolute errors of the approximation. 


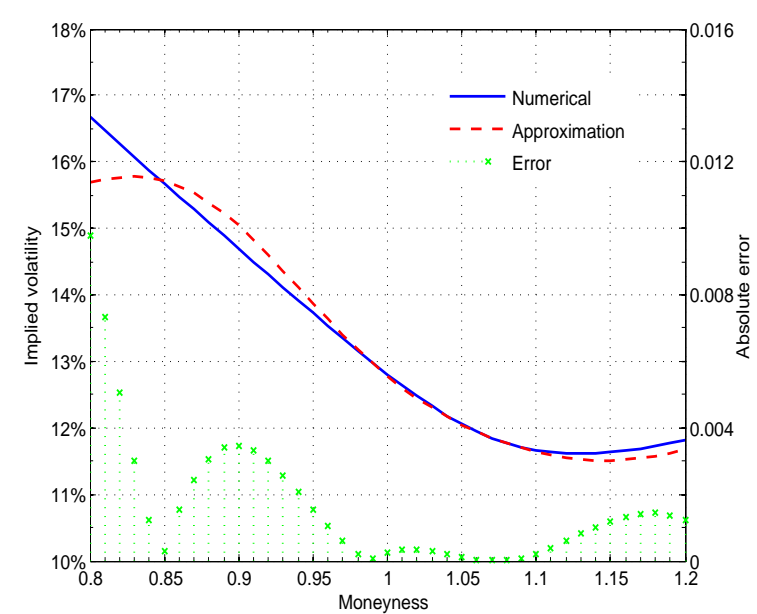

(a) VG dynamics

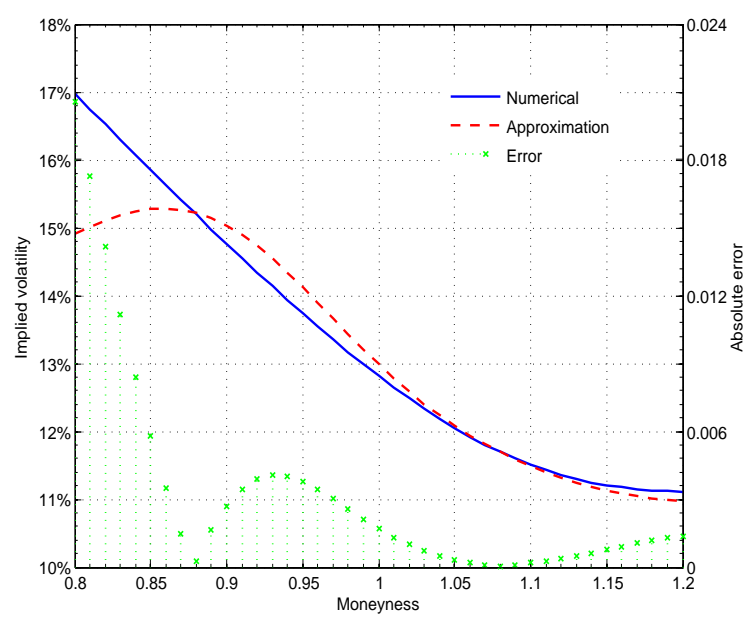

(b) NIG dynamics

Figure 1.3: Approximation of implied volatility for time to maturity fixed to $T=1 / 2$ year.

0.1213, $\nu=0.1686$ and $\theta=-0.1436$ presented in Madan et al. (1998), while in the NIG case the parameters ${ }^{2} \alpha=28.4214, \beta=-15.0862$ and $\delta=0.3196$ from Këllezi and Webber (2004) are used. To compute the true option prices or implied volatilities, we use an efficient Fourier inversion algorithm based on the Lewis-Lipton approach (Lewis (2001), Lipton (2002)). ${ }^{3}$

Figure 1.2 illustrates the numerical errors (in absolute terms) between the true volatilities and the approximated ones. Overall, it seems that the approximation works somewhat better for VG prices compared to NIG prices, at least for the given parameters. In both cases, however, we generally observe a pattern of diminishing approximation error as time to maturity increases. In addition, the validity of the approximation increases in the strike dimension for larger time to maturity. In fact, for very short time to maturity the secondorder approximation of the left wing of the volatility surface deteriorates, especially for more than $10 \%-15 \%$ in-the-money options.

Next, we zoom in on six months to maturity and consider call options in the range of $20 \%$ in and out of the money. Implied volatilities are plotted against moneyness $\bar{K} \equiv K / S_{0}$ in Figure 1.3.2. Under VG dynamics, the approximation does a very good

\footnotetext{
${ }^{2}$ The parameter set was obtained by moment-matching.

${ }^{3}$ An alternative fast Fourier transform (FFT) procedure can be found in Carr and Madan (1999), while Schmelzle (2010) provides a comprehensive overview of Fourier transform methods with applications to option pricing.
} 
job mimicking the true implied volatilities. The mean absolute error between the true and the approximated implied volatilities is 16 bps. Similar in the NIG case, we observe a decent performance of the approximation, but the deterioration in the left wing for short-maturity in-the-money options is more pronounced. In this case, the mean absolute error is 32 bps.

In general, it is not possible to put a bound on the error term associated with the Taylor series expansion due to the fact that some Greeks of a European call option may be unbounded. The Gamma, for instance, becomes unbounded as the total variance $V$ goes to zero. However, Table 1.1 provides a numerical analysis of the error associated with the VG approximation. We investigate how the accuracy of the approximation depends on the parameter values of the VG model and report mean absolute errors for both half-ayear and one-year options. One observes that the approximation is particularly sensitive to the $\sigma$ and $\nu$ parameters. Specifically, the quality of the approximation deteriorates with low levels of volatility $(\sigma<0.10)$ and high excess kurtosis $(\nu>0.25)$. However, the approximation errors are well within the bid-ask spreads observed in the market. A similar analysis may be carried out for the NIG approximation.

Finally, we conduct a calibration experiment based on the approximation. Prices of vanilla calls are generated from a known distribution, i.e., from the VG model and the NIG model. Next, we calibrate to the generated option prices by minimizing the sum of squared errors between the prices and the second-order approximation of the prices. In particular, we study two calibration trials. In Calibration I, we calibrate to a cross-section of 156 call prices with expiries from 3 months to 3 years and relative strikes from $30 \%$ in the money to $30 \%$ out of the money. Specifically, we calibrate over

$$
\begin{gathered}
T \in(0.25,0.5,0.75,1.0,1.25,1.5,1.75,2.0,2.25,2.5,2.25,3.0), \\
\bar{K} \in(0.7,0.75,0.8,0.85,0.9,0.95,1.0,1.05,1.1,1.15,1.2,1.25,1.3) .
\end{gathered}
$$

In Calibration II we decrease the relative strike range to $20 \%$ in and out of the money. The maturities are kept the same which leaves us with a cross-section 108 call prices to calibrate to in this case. The calibrated parameters are provided in Table 1.2 together with the true parameters of the distribution from which the option prices were generated in the first place

The experiment shows that calibration based on the approximation produces parameters that are reasonably close to the true parameters. However, one also observes that the optimal parameters seem to deviate more for calibration I than for II, especially for the 
Table 1.1: Error analysis of the VG approximation

\begin{tabular}{lrrrrr}
\hline \hline Parameter $\sigma$ & \multicolumn{5}{c}{0.20} \\
\hline Abs. change in $\sigma$ & -0.10 & -0.05 & 0.00 & +0.05 & +0.10 \\
MAE $^{\mathrm{a}}(T=0.5)$ & 19.78 & 8.95 & 7.21 & 5.09 & 5.15 \\
MAE $^{\mathrm{a}}(T=1.0)$ & 6.60 & 3.13 & 1.55 & 1.22 & 1.51 \\
\hline Parameter $\nu$ & & & 0.15 & & \\
\hline Abs. change in $\nu$ & -0.10 & -0.05 & 0.00 & +0.05 & +0.10 \\
MAE $^{\mathrm{a}}(T=0.5)$ & 0.97 & 3.72 & 7.21 & 13.55 & 19.42 \\
MAE $^{\mathrm{a}}(T=1.0)$ & 0.20 & 0.79 & 1.55 & 3.09 & 4.73 \\
\hline Parameter $\theta$ & & & -0.15 & & \\
\hline Abs. change in $\theta-0.10$ & -0.05 & 0.00 & +0.05 & +0.10 \\
MAE $^{\mathrm{a}}(T=0.5)$ & 11.47 & 8.35 & 7.21 & 5.02 & 4.24 \\
MAE $^{\mathrm{a}}(T=1.0)$ & 5.31 & 2.84 & 1.55 & 0.95 & 0.87 \\
\hline \hline
\end{tabular}

${ }^{\mathrm{a}}$ Mean absolute error in basis points between the true and the approximated implied volatility curves, computed for a strike range of $K \in[80 ; 120]$ and a spot price $S_{0}=100$. The base case parameters of the VG model are $\sigma=0.20, \nu=0.15, \theta=-0.15$.

Table 1.2: Calibration experiment under Lévy dynamics

\begin{tabular}{|c|c|c|c|c|c|c|}
\hline & \multicolumn{2}{|c|}{ True model } & \multicolumn{2}{|c|}{ Calibration I } & \multicolumn{2}{|c|}{ Calibration II } \\
\hline & VG & NIG & VG & NIG & VG & NIG \\
\hline$\sigma$ & 0.1213 & - & 0.1224 & - & 0.1223 & - \\
\hline$\nu$ & 0.1686 & - & 0.1648 & - & 0.1596 & - \\
\hline$\theta$ & -0.1436 & - & -0.1370 & - & -0.1399 & - \\
\hline$\alpha$ & - & 28.4214 & - & 21.5314 & - & 25.5346 \\
\hline$\beta$ & - & -15.0862 & - & -9.4843 & - & -12.2494 \\
\hline$\delta$ & - & 0.3169 & - & 0.2843 & - & 0.3127 \\
\hline Distance $^{\mathrm{a}}$ & - & - & $8.3 \mathrm{e}-4$ & $2.9 \mathrm{e}-3$ & $7.2 \mathrm{e}-4$ & $1.2 \mathrm{e}-3$ \\
\hline Speed-up ${ }^{b}$ & - & - & 36.0 & 41.5 & 65.0 & 53.0 \\
\hline
\end{tabular}

$\overline{{ }^{a} \text { Error distance between the true volatility surface and the volatility surface based on the }}$ calibrated parameters measured as the root mean square error (RMSE).

${ }^{\mathrm{b}}$ Relative CPU time of calibration based on the approximation versus Fourier inversion. The calibration experiment was implemented in a MatLab environment on a standard PC with $2.53 \mathrm{GHz}$ CPU and $4.00 \mathrm{~GB}$ RAM. 
NIG model. The majority of the calibration errors are due to the wings in the strike dimension for short maturities. As noted, the second-order approximation does not capture these too well under the given parameters, especially for significant in-the-money options. This explains why the calibration performance in calibration I is somewhat worse than in calibration II. Fortunately, the moneyness of calibration instruments is generally not extreme and significantly less extreme for short maturities. In addition, the distance between the true implied volatility surface and the volatility surface computed based on the calibrated parameters is less than one third of a volatility point (30 bps) in all trials. Thus, for the considered cases, the approximation seems appropriate as a first-order calibration approach or at least to provide smart initial values for a more accurate but time-consuming calibration procedure. The approximation-based calibration results in a significant speed-up in computational time compared to calibration based on Fourier inversion. On average it is more than 30 times faster than Fourier inversion. This is only natural as the approximation is simply a sum of elementary functions. Moreover, the moments that enter the approximation are constant across strikes for a fixed maturity and can be safely cached.

\subsection{Expansions under Lévy-Stochastic Volatility Dynamics}

It is well known that exponential Lévy models are able to capture the implied volatility smile or skew for a single maturity, but they are not flexible enough to accommodate the term structure of volatility, especially for long-term options. On the other hand, models allowing for stochastic volatility can generate realistic implied volatilities across maturities but fail to reproduce strong skews and smiles at short maturities. To depict the volatility surface in the strike as well as in the maturity dimensions, a variety of models displaying both jumps and stochastic volatility has been suggested in the literature. Carr et al. (2003) propose the Lévy-Stochastic Volatility process (for short, Lévy-SV process), where the driving source of randomness $X$ is constructed by subordinating a general Lévy process $L$ to an absolutely continuous random clock $Y$

$$
X:=\left(L_{Y_{t}}\right)_{t \geq 0} \quad \text { with } \quad Y_{t}=\int_{0}^{t} y_{s} d s
$$


where the instantaneous activity rate $y$ is given by some positive process independent of $L$.

In a similar manner we can obtain models belonging to the Lévy-SV class within the general framework of Section 1.2 by specifying the total variance process $V$ as a subordinator $Z$ evaluated at an independent and absolutely continuous stochastic time $Y$

$$
V:=\left(Z_{Y_{t}}\right)_{t \geq 0} \quad \text { with } \quad Y_{t}=\int_{0}^{t} y_{s} d s
$$

In this case the differential triplet $\left(b^{V}, 0, F^{V}\right)$ and the cumulant process $K^{V}$ of $V$ are given by

$$
b_{t}^{V}=\beta^{Z} y_{t}, \quad F_{t}^{V}(d x)=\nu^{Z}(d x) y_{t}, \quad \text { and } \quad K_{t}^{V}(\zeta)=\kappa^{Z}(\zeta) Y_{t},
$$

where $\left(\beta^{Z}, 0, \nu^{Z}\right)$ denotes the Lévy-Kintchine triplet of $Z$, and $\kappa^{Z}$ is theLévy-Kintchine exponent given in (1.16). Proposition 1 yields the triplet $\left(b^{X}, c^{X}, F^{X}\right)$ for the corresponding process $X$ in (1.3) as follows

$$
b_{t}^{X}=\beta^{L} y_{t}, \quad c_{t}^{X}=\gamma^{L} y_{t}, \quad F_{t}^{X}(d x)=\nu^{L}(d x) y_{t},
$$

where $\left(\beta^{L}, \gamma^{L}, \nu^{L}\right)$ is the Lévy-Kintchine triplet given in (1.17). Therefore, the driving process $X$ admits the Lévy-SV process representation (1.26) with $L$ being the Lévy process characterized by $\left(\beta^{L}, \gamma^{L}, \nu^{L}\right)$.

The Laplace transform of the stochastic clock $Y$ at a given maturity $T$ is given by

$$
\mathcal{L}_{T}^{Y}(z)=E\left[e^{z Y_{T}}\right]
$$

and is defined in the domain $\mathcal{D}_{T}^{Y}=\left\{z \in \mathbb{C}: E\left[\left|e^{z Y_{T}}\right|\right]<\infty\right\}$. In virtue of the independence between $Z$ and $Y$ in (1.27), the joint Laplace transform $\mathcal{L}_{T}$ defined in (1.6) can easily be expressed in terms of $\mathcal{L}_{T}^{Y}$ as follows

$$
\mathcal{L}_{T}(u, v)=E\left[e^{\left(u \kappa^{Z}(c)+\kappa^{Z}(v)\right) Y_{T}}\right]=\mathcal{L}_{T}^{Y}\left(u \kappa^{Z}(c)+\kappa^{Z}(v)\right)
$$

If we assume that there exists $\varepsilon>0$ such that $\kappa(\varepsilon)<\infty$, then Proposition 2 entails that the expansion (1.13) can be truncated at the order $n$ whenever the point $-n \kappa^{Z}(c)+\kappa^{Z}(n c)$ lies in the interior domain $\stackrel{\circ}{\mathcal{D}}_{T}^{Y}$ of $\mathcal{L}_{T}^{Y}$. The relevant moments can then be computed via the differentiation

$$
E\left[V_{T}^{m} \xi_{T}^{k}\right]=\left.\frac{\partial^{m}}{\partial v^{m}} \mathcal{L}_{T}^{Y}\left(u \kappa^{Z}(c)+\kappa^{Z}(v)\right)\right|_{u=-k, v=k c}, \quad 0 \leq k \leq n,
$$


which specialized to the second-order approximation leads to the following expressions

$$
\begin{aligned}
E\left[V_{T}\right] & =E\left[Y_{T}\right] \frac{d}{d \zeta} \kappa^{Z}(0), \\
E\left[V_{T}^{2}\right] & =E\left[Y_{T}^{2}\right] \frac{d}{d \zeta} \kappa^{Z}(0)^{2}+E\left[Y_{T}\right] \frac{d^{2}}{d \zeta^{2}} \kappa^{Z}(0), \\
E\left[V_{T} \xi_{T}\right] & =E\left[Y_{T}\right] \frac{d}{d \zeta} \kappa^{Z}(c), \\
E\left[\xi_{T}^{2}\right] & =\mathcal{L}_{T}^{Y}\left(-2 \kappa^{Z}(c)+\kappa^{Z}(2 c)\right) .
\end{aligned}
$$

\subsubsection{Specific Lévy-stochastic volatility models}

When constructing a model within the Lévy-SV class a key issue is the choice of the instantaneous activity process $y$ defining the stochastic clock $Y$. Popular specifications include the CIR process of Cox et al. (1985), the Ornstein-Uhlenbeck-type process introduced by Barndorff-Nielsen and Shephard (2001), the quadratic class of Leippold and $\mathrm{Wu}$ (2002) and the 3/2 model studied in Itkin and Carr (2010). In all these cases, the Laplace transform $\mathcal{L}_{T}^{Y}$ is available in terms of elementary functions, and the corresponding expansion (1.13) is obtained in closed form via differentiation in (1.28). We focus on the most common choice in the literature which is the CIR specification. The activity process $y$ is then modeled as

$$
d y_{t}=\kappa\left(\eta-y_{t}\right) d t+\lambda \sqrt{y_{t}} d W_{t}
$$

where the parameters $\kappa, \eta$, and $\lambda$ are positive constants and $W$ is a standard Brownian motion. The Laplace transform of the integrated activity process $Y$ is given by

$$
\mathcal{L}_{T}^{Y}(\zeta)=\frac{\exp \left(\frac{\kappa^{2} \eta T}{\lambda^{2}}\right)}{\left(\cosh \frac{\gamma T}{2}+\frac{\kappa}{\gamma} \sinh \frac{\gamma T}{2}\right)^{\frac{2 \kappa \eta}{\lambda^{2}}}} \exp \left(\frac{2 y_{0} \zeta}{\kappa+\gamma \operatorname{coth} \frac{\gamma T}{2}}\right)
$$

where $\gamma=\sqrt{\kappa^{2}-2 \lambda^{2} \zeta}$. Dufresne (2001) gives a thorough analysis of the integrated CIR process and provides alternative methods for the computation of $E\left[Y_{T}^{n}\right]$. The first and the second moment needed in (1.29) for the second-order approximation are

$$
\begin{aligned}
E\left[Y_{T}\right]= & T \eta+\frac{1-e^{-T \kappa}}{\kappa}\left(y_{0}-\eta\right), \\
E\left[Y_{T}^{2}\right]= & -\frac{5 \eta \lambda^{2}}{2 \kappa^{3}}+\frac{y_{0} \lambda^{2}}{\kappa^{3}}+\frac{T \eta \lambda^{2}}{\kappa^{2}}+\frac{2 \eta \lambda^{2} e^{-T \kappa}}{\kappa^{3}}-\frac{y_{0} \lambda^{2} e^{-2 T \kappa}}{\kappa^{3}} \\
& +\frac{\eta \lambda^{2} e^{-2 T \kappa}}{2 \kappa^{3}}-\frac{2 T y_{0} \lambda^{2} e^{-t \kappa}}{\kappa^{2}}+\frac{2 T \eta \lambda^{2} e^{-T \kappa}}{\kappa^{2}}+E\left[Y_{T}\right]^{2}
\end{aligned}
$$


To complete the construction of the concrete Lévy-SV models to be analyzed below, we specify the subordinator $Z$ in (1.27) as either the Gamma-process or the IG-process introduced in Section 1.3.1. The resulting Lévy-SV models are the so-called VGSV and NIGSV models examined in Carr et al. (2003) as the subordinand Lévy process $L$ in (1.26) becomes a VG or a NIG process. Finally, the explicit second-order approximations are

obtained by inserting the corresponding cumulants $\frac{d}{d \zeta} \kappa^{Z}(0), \frac{d^{2}}{d \zeta^{2}} \kappa^{Z}(0)$ and $\frac{d}{d \zeta} \kappa^{Z}(c)$ from Section 1.3 .1 in (1.29).

\subsubsection{Numerical results}

Let us evaluate the quality of the approximation under both VGSV dynamics and NIGSV dynamics. Schoutens et al. (2004) carefully calibrate these two models to a cross-section of call options on the Eurostoxx 50 index. In the sequel we use the parameter estimates they find, i.e., the VGSV parameters are $C=18.0968, G=20.0276, M=26.3971$, $\kappa=1.2145, \eta=0.5501, \lambda=1.7913$, while the NIGSV parameters are $\alpha=16.1975$, $\beta=-3.1804, \delta=1.0867, \kappa=1.2101, \eta=0.5507, \lambda=1.7864$. The initial value $y_{0}$ of the clock is set to one.

In Figure 1.4 the implied volatilities of the approximation (truncated to 2nd order) are compared to the true values computed via Fourier transform inversion. The approximation captures the smile shape of the true volatility surface very well. This is confirmed by looking at the absolute approximation errors provided in Figure 1.5. The approximation errors are on average fairly low and appear to decrease with time to maturity. Moreover, the validity of the approximation opens up in the strike dimension for increasing time to maturity.

Next, Figure 1.6 fixes the time to maturity to six months and plots the approximated implied volatilities against the true implied volatilities. Considering calls with relative strikes in the range of $20 \%$ in and out of the money, we get a mean absolute error of only 15 bps under the VGSV model. While under the NIGSV model, the mean absolute error is $16 \mathrm{bps}$.

In Table 1.3 we carefully study the error of the VGSV approximation and how the accuracy depends on the parameters of the model. The sentitivity to the VG parameters $(C, G, M)$ was investigated in Section 1.3.2 so in the following these are kept fixed. Instead, we analyze how the accuracy of the approximation depends on the CIR parameters $(\kappa, \eta$, $\lambda)$ governing the stochastic volatility in the VGSV model. We report mean absolute errors 


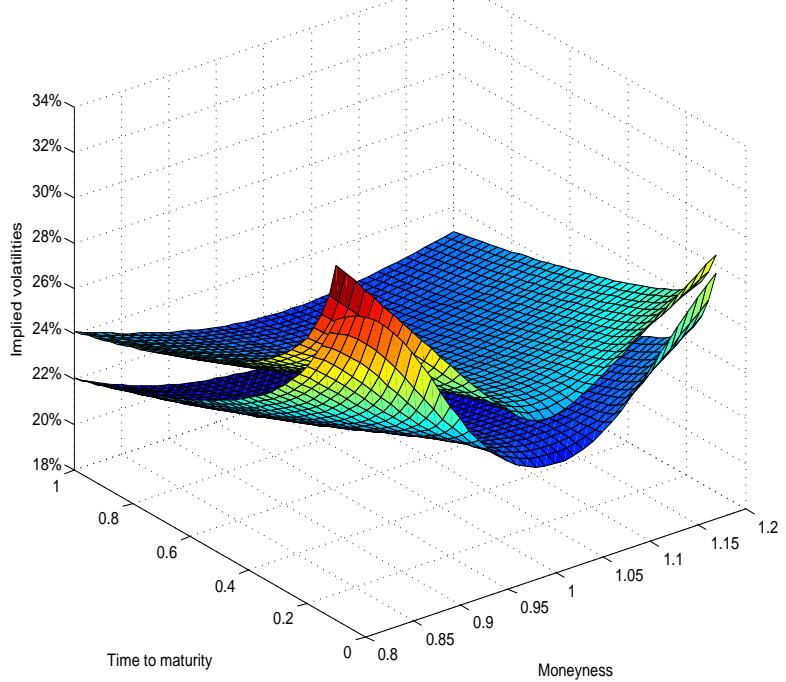

(a) VGSV dynamics

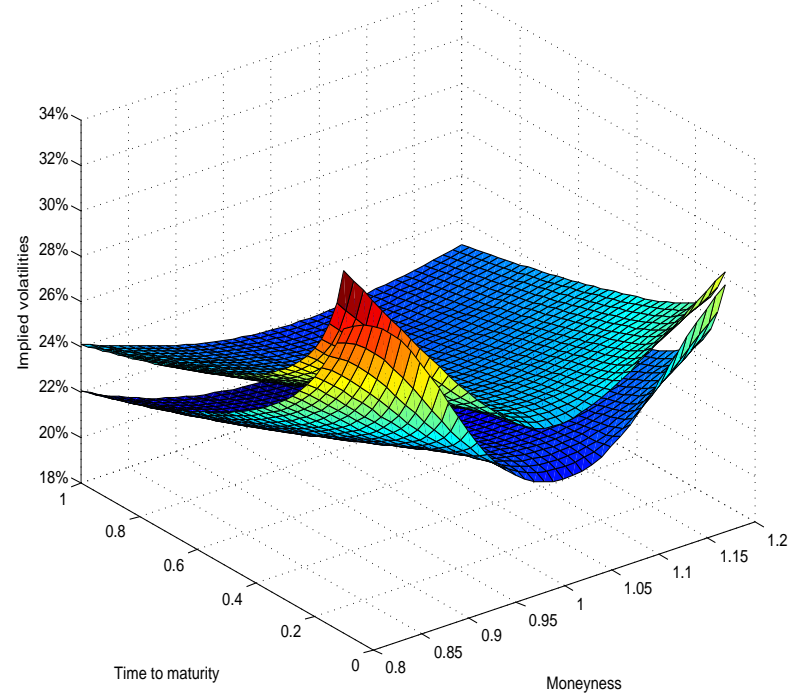

(b) NIGSV dynamics

Figure 1.4: Approximated volatilities (lower surface) against true volatilities (upper surface). The approximate surface is moved down by $200 \mathrm{bps}$ for ease of interpretation.

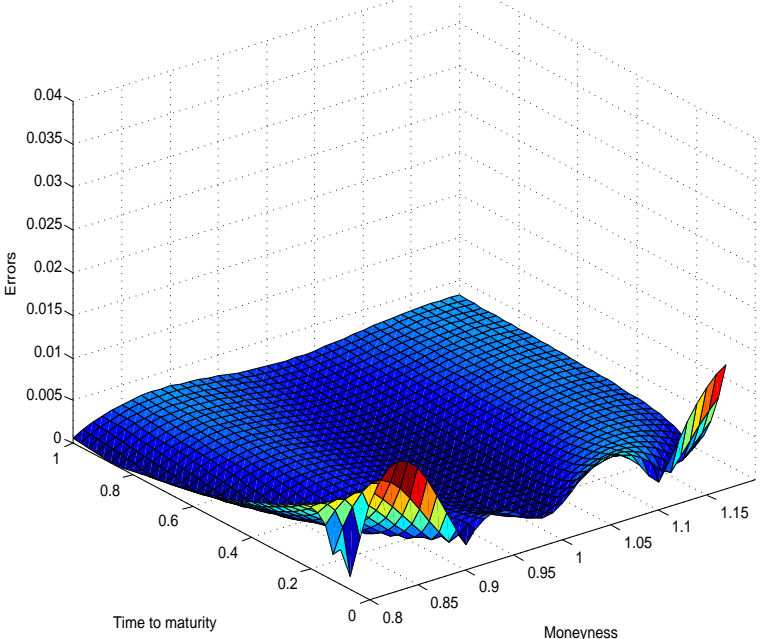

(a) VGSV dynamics

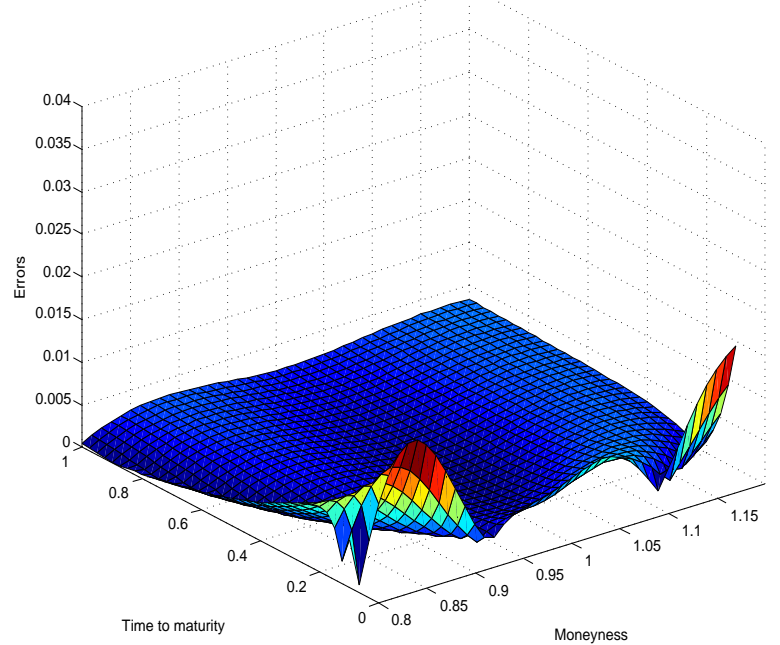

(b) NIGSV dynamics

Figure 1.5: Absolute errors of the approximation. 


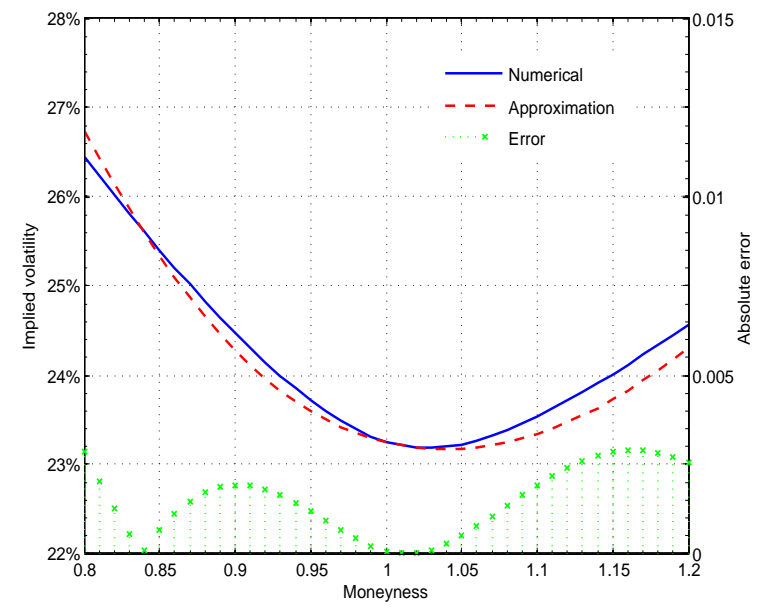

(a) VGSV dynamics

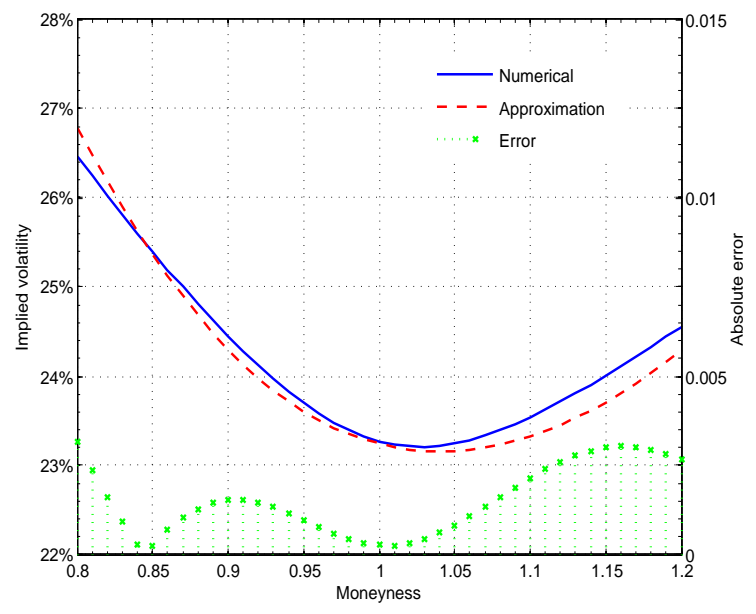

(b) NIGSV dynamics

Figure 1.6: Approximation of implied volatility for time to maturity fixed to $T=1 / 2$ year.

Table 1.3: Error analysis of the VGSV approximation

\begin{tabular}{lrrrrr}
\hline \hline Parameter $\kappa$ & \multicolumn{5}{c}{1.25} \\
\hline Abs. change in $\kappa$ & -0.50 & -0.25 & 0.00 & +0.25 & +0.50 \\
MAE $^{\mathrm{a}}(T=0.5)$ & 9.28 & 8.49 & 7.80 & 7.20 & 6.66 \\
MAE $^{\mathrm{a}}(T=1.0)$ & 16.47 & 13.42 & 10.95 & 8.95 & 7.33 \\
\hline Parameter $\eta$ & & 0.75 & & \\
\hline Abs. change in $\eta$ & -0.50 & -0.25 & 0.00 & +0.25 & +0.50 \\
MAE $^{\mathrm{a}}(T=0.5)$ & 11.47 & 9.39 & 7.80 & 6.52 & 5.51 \\
MAE $^{\mathrm{a}}(T=1.0)$ & 18.57 & 14.06 & 10.95 & 8.73 & 7.09 \\
\hline Parameter $\lambda$ & & & 1.50 & & \\
\hline Abs. change in $\lambda-0.50$ & -0.25 & 0.00 & +0.25 & +0.50 \\
MAE $^{\mathrm{a}}(T=0.5)$ & 3.11 & 4.96 & 7.80 & 11.98 & 18.12 \\
MAE $^{\mathrm{a}}(T=1.0)$ & 2.17 & 5.34 & 10.95 & 18.34 & 30.22 \\
\hline \hline a Mean absere
\end{tabular}

${ }^{\mathrm{a}}$ Mean absolute error in basis points between the true and the approximated implied volatility curves, computed for a strike range of $K \in[80 ; 120]$ and a spot price $S_{0}=100$. The base case parameters of the VGSV model are $C=18, G=20, M=26, \kappa=1.25, \eta=$ $0.75, \lambda=1.50$. 
Table 1.4: Calibration experiment under Lévy-SV dynamics

\begin{tabular}{|c|c|c|c|c|c|c|}
\hline & \multicolumn{2}{|c|}{ True model } & \multicolumn{2}{|c|}{ Calibration I } & \multicolumn{2}{|c|}{ Calibration II } \\
\hline & VGSV & NIGSV & VGSV & NIGSV & VGSV & NIGSV \\
\hline$C$ & 18.0968 & - & 18.0088 & - & 18.5002 & - \\
\hline$G$ & 20.0276 & - & 20.3702 & - & 20.4697 & - \\
\hline$M$ & 26.3971 & - & 25.8480 & - & 26.7156 & - \\
\hline$\alpha$ & - & 16.1975 & - & 16.2786 & - & 16.2464 \\
\hline$\beta$ & - & -3.1804 & - & -2.7329 & - & -2.9643 \\
\hline$\delta$ & - & 1.0867 & - & 1.0979 & - & 1.0841 \\
\hline$\kappa$ & 1.2145 & 1.2101 & 1.1938 & 1.1913 & 1.1130 & 1.1123 \\
\hline$\eta$ & 0.5501 & 0.5507 & 0.5679 & 0.5688 & 0.5900 & 0.5894 \\
\hline$\lambda$ & 1.7913 & 1.7864 & 1.6440 & 1.6437 & 1.7332 & 1.7864 \\
\hline Distance $^{\mathrm{a}}$ & - & - & $2.3 \mathrm{e}-3$ & $2.4 \mathrm{e}-3$ & $3.2 \mathrm{e}-3$ & $3.2 \mathrm{e}-3$ \\
\hline Speed-up ${ }^{b}$ & - & - & 60.8 & 58.4 & 69.8 & 61.5 \\
\hline
\end{tabular}

${ }^{a}$ Error distance between the true volatility surface and the volatility surface based on the calibrated parameters measured as the root mean square error (RMSE).

${ }^{\mathrm{b}}$ Relative CPU time of calibration based on the approximation versus Fourier inversion. The calibration experiment was implemented in a MatLab environment on a standard PC with $2.53 \mathrm{GHz}$ CPU and 4.00 GB RAM.

for both half-a-year and one-year options. The VGSV approximation appears particularly sensitive to the $\lambda$ parameter and the quality of the approximation deteriorates for high vol-of-vol $(\lambda>2.0)$. Also, slight deterioration emerges for low mean reversion $(\kappa<0.5)$ and low long-run mean $(\eta<0.25)$ of the stochastic clock. Nevertheless, the errors are within bid-ask spreads. A similar analysis may be executed for the NIGSV model.

Finally, we investigate the performance of approximation-based calibration under stochastic volatility Lévy dynamics. We consider the same calibration experiment outlined in Section 1.3.2. Table 1.4 summarizes the results of the calibration experiments. We obtain parameter estimates that are very close to the true ones in both calibration trials and under the two model assumptions. This confirms the good approximation of the volatility surface that we saw above based on the parameters from Schoutens et al. (2004). Looking at the distance between the true implied volatility surface and the volatility surface computed based on the calibrated parameters, the mean error is less than one third of a volatility point (33 bps) in all cases. Compared to calibration I, calibration 
II is slightly worse. This is probably due to the more sparse information contained in this sample. Finally, the approximation-based calibration is more than 50 times faster than calibration based on Fourier inversion, on average. Again, this speed-up can be contributed to the facts that the approximation is simply a sum of elementary functions and the moments that appear in the approximation only need to be computed once for every maturity.

\subsection{Expansions under Affine Dynamics}

Although the Lévy-SV models examined above have proved successful in depicting both the skew and the term structure of the volatility surface, they do not quite encompass the well-documented leverage effect, i.e., the negative correlation between asset returns and their volatilities. This stylized fact can be accounted for by letting the innovations of the subordinator $Z$ be correlated with the innovations of the random clock $y$ in the construction (1.27) of the total variance process. The framework of affine processes introduced by Duffie et al. (2000) and further developed by Duffie et al. (2003) offers a natural way to incorporate this correlation. We refer to Kallsen (2006) for a very comprehensive account of affine processes. For the reader's convenience we collect a few fundamental properties in Appendix 1.7.2.

Here, we model the total variance process $V$ as one of the components of a rather general bivariate affine process $(y, V)$ taking values on $\mathbb{R}_{+}^{2}$ and differential triplet $\left(b^{y V}, c^{y V}, F^{y V}\right)$ given by

$$
b_{t}^{y V}=\beta^{0}+\beta^{1} y_{t}, \quad c_{t}^{y V}=\gamma^{0}+\gamma^{1} y_{t}, \quad F_{t}^{y V}=\nu^{0}+\nu^{1} y_{t},
$$

where $\left(\beta^{j}, \gamma^{j}, \nu^{j}\right), j=0,1$ are admissible Lévy triplets on $\mathbb{R}^{2}$. To ensure that the pair is positive, $V$ is increasing, and no explosions occur in finite time, we assume that $\left(\beta^{j}, \gamma^{j}, \nu^{j}\right)$, $j=0,1$ meet the following requirements

$$
\begin{aligned}
& \beta_{i}^{0} \geq 0 \quad \text { for } i=1,2, \quad \text { and } \quad \beta_{2}^{1} \geq 0 \\
& \nu^{j}\left(\left(\mathbb{R}_{+}^{2}\right)^{c}\right)=0 \quad \text { for } j=0,1 \\
& \int_{\mathbb{R}_{+}} x_{i} \nu^{j}\left(d x_{1}, d x_{2}\right)<+\infty \quad \text { for } j=0,1, \quad i=1,2 \\
& \gamma^{0}=0 \quad \text { and } \quad \gamma^{1}=\left(\begin{array}{ll}
\gamma & 0 \\
0 & 0
\end{array}\right) \quad \text { with } \gamma \geq 0 .
\end{aligned}
$$


The joint cumulant process $K^{y V}$ of $(y, V)$ is given by

$$
K_{t}^{y V}\left(\zeta_{1}, \zeta_{2}\right)=\kappa^{0}\left(\zeta_{1}, \zeta_{2}\right) t+\kappa^{1}\left(\zeta_{1}, \zeta_{2}\right) Y_{t} \quad \text { with } \quad Y_{t}=\int_{0}^{t} y_{s} d s,
$$

where $\kappa^{j}$ denotes the Lévy exponent of the Lévy triplet $\left(\beta^{j}, \gamma^{j}, \varphi^{j}\right)$, i.e.,

$$
\begin{aligned}
\kappa^{0}\left(\zeta_{1}, \zeta_{2}\right) & =\beta_{1}^{0} \zeta_{1}+\beta_{2}^{0} \zeta_{2}+\int_{\mathbb{R}_{+}^{2}}\left(e^{\zeta_{1} x_{1}+\zeta_{2} x_{2}}-1\right) \nu^{0}\left(d x_{1}, d x_{2}\right) \\
\kappa^{1}\left(\zeta_{1}, \zeta_{2}\right) & =\beta_{1}^{1} \zeta_{1}+\beta_{2}^{1} \zeta_{2}+\frac{1}{2} \gamma \zeta_{2}^{1}+\int_{\mathbb{R}_{+}^{2}}\left(e^{\zeta_{1} x_{1}+\zeta_{2} x_{2}}-1\right) \nu^{1}\left(d x_{1}, d x_{2}\right) .
\end{aligned}
$$

Clearly, the cumulant process $K^{V}$ of the total variance process $V$ is given by

$$
K_{t}^{V}(\zeta)=K_{t}^{y V}(0, \zeta)=\kappa^{0}(0, \zeta) t+\kappa^{1}(0, \zeta) Y_{t}
$$

We can describe the pair $(y, V)$ in a less rigorous but perhaps more intuitive manner as follows

$$
\begin{aligned}
& d y_{t}=\left(\beta_{1}^{0}+\beta_{1}^{1} y_{t}\right) d t+d J_{t}^{0}+d\left(J_{Y_{t}}^{1}\right)+\sqrt{\gamma y_{t}} d B_{t} \\
& d V_{t}=\left(\beta_{2}^{0}+\beta_{2}^{1} y_{t}\right) d t+d Z_{t}^{0}+d\left(Z_{Y_{t}}^{1}\right) \\
& d Y_{t}=y_{t} d t
\end{aligned}
$$

where $\left(J^{0}, Z^{0}\right)$ and $\left(J^{1}, Z^{1}\right)$ are bivariate drift-less subordinators, independent of each other and described by the Lévy measures $\nu^{0}$ and $\nu^{1}$, while $B$ is a Brownian motion independent of anything else. The dynamics (1.36) highlight that if $\beta_{2}^{0}=0$ and $Z^{0} \equiv 0$, the total variance process admits the representation as the time-changed process

$$
V_{t}=Z_{Y_{t}} \quad \text { with } \quad Z_{t}=\beta_{2}^{1} t+Z_{t}^{1}
$$

However, in contrast to the time-change construction presented in Section 1.4, the total variance $V$ and its rate of time-change $y$ are correlated whenever the subordinators $Z^{1}$ and $J^{1}$ are correlated.

The affine structure for the pair $(y, V)$ carries over to all the relevant elements of the asset pricing model (1.5) constructed upon it. Noticeably, the pair $(y, \log M(\zeta))$ with $M(\zeta)$ defined in (1.2) and $\zeta \leq 0$ is affine with defining Lévy triplets $\left(\bar{\beta}^{j}, \bar{\gamma}^{j}, \bar{\nu}^{j}\right)$ given by

$$
\bar{\beta}^{j}=\left(\begin{array}{c}
\beta_{1}^{j} \\
-\kappa^{j}(0, \zeta)+\zeta \beta_{2}^{j}
\end{array}\right), \quad \bar{\gamma}^{j}=\gamma^{j}, \quad \bar{\nu}^{j}(B)=\int_{\mathbb{R}_{+}^{2}} 1_{B}\left(x_{1}, \zeta x_{2}\right) \nu^{j}\left(d x_{1}, d x_{2}\right),
$$


for $j=0,1$ and $\left(\bar{\beta}^{2}, \bar{\gamma}^{2}, \bar{\nu}^{2}\right)=(0,0,0)$. As a result, Assumption 1 is automatically satisfied in virtue of the integrability requirement (1.32) and Proposition 6 in Appendix 1.7.2.

Proceeding along the lines of Proposition 1, we obtain that the pair $(y, X)$ driving the asset price dynamics (1.5) is an affine process taking values on $\mathbb{R}_{+} \times \mathbb{R}$. The defining Lévy triplets $\left(\widetilde{\beta}^{j}, \widetilde{\gamma}^{j}, \widetilde{\nu}^{j}\right), j=0,1,2$ are given by

$$
\begin{aligned}
& \widetilde{\beta}_{1}^{j}=\beta_{1}^{j}, \quad \widetilde{\beta}_{2}^{j}=\theta \beta_{2}^{j}+\int_{\mathbb{R}_{+}^{2}} \nu^{j}\left(d x_{1}, d x_{2}\right) \int_{\mathbb{R}} h(w) \mu\left(d w ; \theta x_{2}, x_{2}\right), \\
& \widetilde{\gamma}^{0}=\left(\begin{array}{cc}
0 & 0 \\
0 & \beta_{2}^{0}
\end{array}\right), \quad \widetilde{\gamma}^{1}=\left(\begin{array}{cc}
\gamma & 0 \\
0 & \beta_{2}^{1}
\end{array}\right) \\
& \widetilde{\nu}^{j}\left(B_{1} \times B_{2}\right)=\int_{\mathbb{R}_{+}^{2}} 1_{B_{1}}\left(x_{1}\right) \mu\left(B_{2} ; \theta x_{2}, x_{2}\right) \nu^{j}\left(d x_{1}, d x_{2}\right)
\end{aligned}
$$

for $j=0,1$, while $\left(\widetilde{\beta}^{2}, \widetilde{\gamma}^{2}, \widetilde{\nu}^{2}\right)=(0,0,0)$. We note that if $\beta_{2}^{0}=0$ and $Z^{0} \equiv 0$, the timechange representation (1.37) of the total variance $V$ transfers to the $X$ process which is described by the following heuristic dynamics

$$
\begin{aligned}
& d y_{t}=\left(\beta_{1}^{0}+\beta_{1}^{1} y_{t}\right) d t+d J_{t}^{0}+d\left(J_{Y_{t}}^{1}\right)+\sqrt{\gamma y_{t}} d B_{t} \\
& d X_{t}=d\left(L_{Y_{t}}\right) \\
& d Y_{t}=y_{t} d t
\end{aligned}
$$

where $\left(J^{1}, L\right)$ is the bivariate Lévy process characterized by the triplet $\left(\widetilde{\beta}^{1}, \widetilde{\gamma}^{1}, \widetilde{\nu}^{1}\right)$ and $J^{0},\left(J^{1}, L\right)$ and $B$ are independent. Under suitable integrability conditions, the covariation between the innovations of $X$ and $y$ is given by

$$
d\left\langle J_{t}^{1}, L_{t}\right\rangle=\int_{\mathbb{R}_{+} \times \mathbb{R}} x_{1} x_{2} \widetilde{\nu}^{1}\left(d x_{1}, d x_{2}\right)=\theta \int_{\mathbb{R}_{+}^{2}} x_{1} x_{2} \nu^{1}\left(d x_{1}, d x_{2}\right)=\theta d\left\langle J_{t}^{1}, Z_{t}^{1}\right\rangle .
$$

Since the underlying assumption for the skewness parameter is $\theta<-\frac{1}{2}$, we see that a positive correlation between $y$ and $V$ translates into a negative correlation, the leverage effect, between the asset returns driven by $X$ and the volatility $y$.

Let us now turn to the joint Laplace transform $\mathcal{L}_{T}$ defined in (1.6).

Proposition 3. The transform $\mathcal{L}_{T}$ is given by

$$
\mathcal{L}_{T}(u, v)=\exp \left(A_{T}(u, v)+B_{T}(u, v) y_{0}\right), \quad \forall(u, v) \in \mathbb{C}^{+} \times \mathbb{C}^{-}
$$


where the functions $A$ and $B$ solve the Riccati-type equations

$$
\begin{aligned}
& \frac{d}{d t} A_{t}(u, v)=\kappa^{0}\left(B_{s}(u, v), v\right)+u \kappa^{0}(0, c), \\
& \frac{d}{d t} B_{t}(u, v)=\kappa^{1}\left(B_{t}(u, v), v\right)+u \kappa^{1}(0, c),
\end{aligned}
$$

with $\kappa^{0}$ and $\kappa^{1}$ given by (1.34) and (1.35) and initial conditions $A_{0}(u, v)=B_{0}(u, v)=0$.

Proof. The three-variate process $\left(y, V, K^{V}(c)\right)$ is affine with defining triplets $\left(\widehat{\beta}^{j}, \widehat{\gamma}^{j}, \widehat{\nu}^{j}\right)$ given by

$$
\widehat{\beta}^{j}=\left(\begin{array}{c}
\beta_{1}^{j} \\
\beta_{2}^{j} \\
\kappa^{j}(0, c)
\end{array}\right), \quad \widehat{\gamma}^{j}=\left(\begin{array}{ccc}
\gamma_{11}^{j} & \gamma_{12}^{j} & 0 \\
\gamma_{21}^{j} & \gamma_{22}^{j} & 0 \\
0 & 0 & 0
\end{array}\right), \quad \widehat{\nu}^{j}(B)=\int 1_{B}\left(x_{1}, x_{2}, 0\right) \nu^{j}\left(d x_{1}, d x_{2}\right),
$$

for $j=0,1$ and $\left(\widehat{\beta}^{j}, \widehat{\gamma}^{j}, \widehat{\nu}^{j}\right)=0$ for $j=2,3$. Applying Theorem 1 in the Appendix 1.7.2 yields the result.

For some specifications of $\kappa^{0}$ and $\kappa^{1}$ the ODEs above can be solved analytically. The characteristic function (1.7) is then available in closed-form and Fourier transform methods become a feasible technology for option pricing and model calibration. However, in most cases the solution of (1.40) and (1.41) needs to be found numerically. Fourier transform inversion is a numerical integration problem which involves hundreds of evaluations of $\mathcal{L}_{T}$, each of them requiring the numerical solution of a system of ODEs. While a single price computation for a given parameter set may seem amenable, the method becomes computationally too slow and not optimal for calibration purposes (see e.g. Glasserman and $\operatorname{Kim}$ (2009) for a discussion on this issue). Typically, the expansion moments $E\left[V_{T}^{m} \xi_{t}^{k}\right]$ are not available in closed-form either, as the differentiation required in (1.14) cannot be carried out explicitly except in those cases when $\mathcal{L}_{T}$ can be computed analytically. Nevertheless, differentiation yields the following useful result.

Proposition 4. Assume that the functions $A$ and $B$ solving (1.40) and (1.41) have an analytical continuation on an open set $U \subset \mathbb{C}^{d}$ with $0 \in U$. Then the moments $E\left[V_{T}^{m} e^{u K_{T}^{V}(c)+v V_{T}}\right]$ are finite for all $(u, v) \in U \cap \mathbb{R}^{2}, m \geq 0$ and they are given by

$$
E\left[V_{T}^{m} e^{u K_{T}^{V}(c)+v V_{T}}\right]=\mathcal{L}_{T}^{(m)}(u, v)
$$


where we have employied the notation $\mathcal{L}_{T}^{(m)}(u, v)=\frac{\partial^{m}}{\partial v^{m}} \mathcal{L}_{T}(u, v)$. Furthermore, the derivatives $\mathcal{L}_{T}^{(m)}(u, v)$ can be computed via the recursive scheme

$$
\begin{aligned}
\mathcal{L}_{T}^{(0)}(u, v) & =\mathcal{L}_{T}(u, v)=\exp \left(A_{T}^{(0)}(u, v)+B_{T}^{(0)}(u, v) y_{0}\right), & & m=0, \\
\mathcal{L}_{T}^{(m)}(u, v) & =\sum_{i=0}^{m-1}\left(\begin{array}{c}
m-1 \\
i
\end{array}\right) \mathcal{L}_{T}^{(i)}(u, v)\left(A_{T}^{(m-i)}(u, v)+B_{T}^{(m-i)}(u, v) y_{0}\right), & & m \geq 1,
\end{aligned}
$$

where $A_{t}^{(i)}(u, v), B_{t}^{(i)}(u, v), i=0, \ldots, m$ satisfy the following system of ODEs

$$
\begin{aligned}
\frac{d}{d t} A_{t}^{(0)}(u, v) & =\kappa^{0}\left(B_{t}^{(0)}(u, v), v\right)+u \kappa^{0}(0, c), \\
\frac{d}{d t} B_{t}^{(0)}(u, v) & =\kappa^{1}\left(B_{t}^{(0)}(u, v), v\right)+u \kappa^{1}(0, c), \\
\frac{d}{d t} A_{t}^{(i)}(u, v) & =\frac{\partial^{i}}{\partial v^{i}}\left(\kappa^{0}\left(B_{t}^{(0)}(u, v), v\right)\right), \\
\frac{d}{d t} B_{t}^{(i)}(u, v) & =\frac{\partial^{i}}{\partial v^{i}}\left(\kappa^{1}\left(B_{t}^{(0)}(u, v), v\right)\right),
\end{aligned}
$$

with $\kappa_{0}$ and $\kappa_{1}$ given by (1.34) and (1.35) and initial conditions $A_{0}^{(i)}(u, v)=B_{0}^{(i)}(u, v)=0$ for $i=0, \ldots, m$.

Proof. Theorem 2 in Appendix 1.7.2 ensures that the Laplace transform $\mathcal{L}_{T}$ is given by (1.39) not only for $(u, v) \in \mathbb{C}^{+} \times \mathbb{C}^{-}$but for all $(u, v) \in \stackrel{\circ}{\mathcal{D}}_{T}$. Successive differentiations of (1.39) with respect to $v$ yield the resulting variational equations.

To sum up, if both $(0,0)$ and $(-n, n c)$ lie in the interior domain $\stackrel{\circ}{\mathcal{D}}_{T}$, the expansion (1.13) can be truncated at the order $n$. The relevant moments and cross-moments are available up to the solution of a system of ODEs which can be computed fast by standard numerical methods. In contrast to Fourier transform methods, only a very limited number of such computations is required by the approximation which then provides a potentially useful alternative for computing option prices in numerically demanding problems such as calibration.

The second-order approximation requires the following quantities

$$
\begin{aligned}
E\left[e^{u K_{T}^{V}(c)+v V_{T}}\right] & =\mathcal{L}_{T}(u, v)=\exp \left(A_{T}^{(0)}+B_{T}^{(0)} y_{0}\right), \\
E\left[V_{T} e^{u K_{T}^{V}(c)+v V_{T}}\right] & =\mathcal{L}_{T}(u, v)\left(A_{T}^{(1)}+B_{T}^{(1)} y_{0}\right), \\
E\left[V_{T}^{2} e^{u K_{T}^{V}(c) t+v V_{t}}\right] & =\mathcal{L}_{T}(u, v)\left(\left(A_{T}^{(1)}+B_{T}^{(1)} y_{0}\right)^{2}+\left(A_{T}^{(2)}+B_{T}^{(2)} y_{0}\right)\right),
\end{aligned}
$$


where we have suppressed the common argument $(u, v)$ of the functions $A_{t}^{(i)}(u, v)$ and $B_{t}^{(i)}(u, v)$ which are given as the solution of

$$
\begin{aligned}
\frac{d}{d t} A_{t}^{(0)} & =\kappa^{0}\left(B_{t}^{(0)}, v\right)+u \kappa^{0}(0, c) \\
\frac{d}{d t} B_{t}^{(0)} & =\kappa^{1}\left(B_{t}^{(0)}, v\right)+u \kappa^{1}(0, c) \\
\frac{d}{d t} A_{t}^{(1)} & =\nabla \kappa^{0}\left(B_{t}^{(0)}, v\right) \cdot\left(B_{t}^{(1)}, 1\right)^{\top} \\
\frac{d}{d t} B_{t}^{(1)} & =\nabla \kappa^{1}\left(B_{t}^{(0)}, v\right) \cdot\left(B_{t}^{(1)}, 1\right)^{\top} \\
\frac{d}{d t} A_{t}^{(2)} & =\left(B_{t}^{(1)}, 1\right) \cdot \nabla^{2} \kappa^{0}\left(B_{t}^{(0)}, v\right) \cdot\left(B_{t}^{(1)}, 1\right)^{\top}+\nabla \kappa^{0}\left(B_{t}^{(0)}, v\right) \cdot\left(B_{t}^{(2)}, 0\right)^{\top}, \\
\frac{d}{d t} B_{t}^{(2)} & =\left(B_{t}^{(1)}, 1\right) \cdot \nabla^{2} \kappa^{1}\left(B_{t}^{(0)}, v\right) \cdot\left(B_{t}^{(1)}, 1\right)^{\top}+\nabla \kappa^{1}\left(B_{t}^{(0)}, v\right) \cdot\left(B_{t}^{(2)}, 0\right)^{\top},
\end{aligned}
$$

with initial conditions $A_{0}^{(i)}(u, v)=B_{0}^{(i)}(u, v)=0$ for $i=1,2$.

\subsubsection{Numerical results}

Let us revisit the VGSV model of Section 1.4. As mentioned, the CIR dynamics is just an affine diffusion process. Thus, the VGSV model constitutes a simple case of the general affine model (1.38). Below we conduct a number of calibrations of the VGSV model to study the speed-up obtained with the approximation compared to Fourier inversion.

Assume we have a data matrix of 146 call prices generated from the true distribution with parameters $C=18.0968, G=20.0276, M=26.3971, \kappa=1.2145, \eta=0.5501$, $\lambda=1.7913$. The maturities and relative strikes are given by

$$
\begin{gathered}
T \in(0.25,0.5,0.75,1.0,1.25,1.5,1.75,2.0,2.25,2.5,2.25,3.0), \\
\bar{K} \in(0.7,0.75,0.8,0.85,0.9,0.95,1.0,1.05,1.1,1.15,1.2,1.25,1.3) .
\end{gathered}
$$

Calibration using the approximation (truncated to the 2nd order) or Fourier inversion yields the parameter values given in Table 1.5.

Table 1.6 compares calibration speed-up gains from using the approximation over Fourier inversion for selected initial values in the optimization routine. ${ }^{4}$ In particular, we investigate the speed-up under two scenarios. In the first scenario we use the known

${ }^{4}$ The experiment is implemented in a MatLab environment on a standard PC with $2.53 \mathrm{GHz} \mathrm{CPU}$ and 4.00 GB RAM. 
Table 1.5: Calibrated parameters

\begin{tabular}{lcccccc}
\hline \hline & \multicolumn{6}{c}{ Parameters } \\
\cline { 2 - 7 } Method & $C$ & $G$ & $M$ & $\kappa$ & $\eta$ & $\lambda$ \\
\hline Fourier & 18.09 & 20.03 & 26.40 & 1.22 & 0.55 & 1.79 \\
Approximation & 17.97 & 20.45 & 26.01 & 1.20 & 0.57 & 1.65 \\
\hline \hline
\end{tabular}

Table 1.6: Calibration speed-up by using the approximation compared to Fourier inversion

\begin{tabular}{lcc}
\hline \hline & \multicolumn{2}{c}{ Speed-up $^{\mathrm{b}}$} \\
\cline { 2 - 3 } Initial values $^{\mathrm{a}}$ & Numerical & Explicit \\
\hline$[5,5,5,1,1,1]$ & 177.8 & 80.4 \\
{$[10,10,10,1,1,1]$} & 152.7 & 76.1 \\
{$[15,15,15,1,1,1]$} & 132.3 & 54.0 \\
{$[20,20,20,1,1,1]$} & 116.5 & 50.8 \\
{$[25,25,25,1,1,1]$} & 152.6 & 73.3 \\
\hline \hline a Initial values of the parameters $[C, G, M, \kappa, \eta, \lambda]$ used in \\
the optimization. \\
b Relative CPU time of Fourier-based calibration over \\
approximation-based calibration.
\end{tabular}

explicit characteristic function of the VGSV model, while in the second scenario the characteristic function is only known up to the solution of a system of ODEs. In the latter scenario we use an explicit Runge-Kutta algorithm (Dormand and Prince (1980)) to numerically solve the ODEs.

In terms of speed-up, the approximation-based calibration approach is most attractive in situations where no explicit characteristic function exists. Moreover, one observes that the computational time of the calibration depends crucially on choosing reasonable initial values for the optimization routine. Naturally, the speed-up is much smaller when we choose initial values close to the true parameters than when more naive initial values are used.

From Table 1.5 we observe that approximation-calibrated parameters are just slightly off compared to the true parameters and may work as smart initial values to speed-up 
Table 1.7: Time reduction when using the approximation-based parameters as initial values.

\begin{tabular}{lc}
\hline \hline Initial values $^{\mathrm{a}}$ & CPU time reduction \\
\hline$[5,5,5,1,1,1]$ & $85.0 \%$ \\
{$[10,10,10,1,1,1]$} & $80.3 \%$ \\
{$[15,15,15,1,1,1]$} & $66.2 \%$ \\
{$[20,20,20,1,1,1]$} & $15.3 \%$ \\
{$[25,25,25,1,1,1]$} & $74.2 \%$ \\
\hline \hline Alternative initial values of the parameters \\
{$[C, G, M, \kappa, \eta, \lambda]$ used in the optimization. } \\
b Reduction in CPU time of Fourier-based calibra- \\
tion when using the approximation-based parameters \\
compared to using the alternative initial values.
\end{tabular}

Fourier-based model calibration. This is in particular so when the characteristic function is not known in closed form. In Table 1.7 we compute the percentage computational time reduction from using the smart values compared to calibrations taking naive initial values.

Clearly, compared to using alternative (naive) initial values in the optimization, applying the approximation-based parameters as initial values may result in a significant time-reduction in calibration based on Fourier inversion. Thus, it appears that a viable two-step calibration approach would be to (i) do an approximation-based calibration, and then (ii) do a more accurate Fourier-based calibration taking the optimal parameters from the first calibration as initial values.

\subsection{Conclusion}

We have presented an expansion of option prices in terms of Black-Scholes prices and higher-order Greeks. The expansion is simple, easy to implement, and broadly applicable across different option pricing models based on time-changed Brownian motions. We have shown how to derive expansions for popular models such as the Variance Gamma and Normal Inverse Gaussian as well as their stochastic volatility counterparts. Also, we have demonstrated how our expansion approach can be extended to a more general subclass of affine jump-diffusion models for stochastic volatility.

The expansion decomposes the option price into a series of adjustment terms associated 
with higher-order option risks. It clarifies how these risks are priced across different option pricing models and shows how the risk adjustments are directly related to the features and parameters of the particular model at hand. This is the primary advantage of the expansion and allows for a much more transparent approach to option pricing in sophisticated models.

Finally, our numerical results indicate that the proposed expansion may be used for fast, first-order model calibration or at least to provide smart initial conditions for the optimization problem. This is particularly attractive to more general affine models without closed-form pricing transforms which would otherwise be considered analytically nontractable.

\subsection{Appendix}

\subsubsection{Differential Triplets and Cumulant Processes}

In this appendix we report some basic facts on semimartingale characteristics and cumulant processes which are relevant for this work. A detailed and rigorous analysis is provided in Kallsen (2006) and Kallsen and Shiryaev (2002).

Let $X=\left(X^{1}, \ldots, X^{d}\right)$ be a $\mathbb{R}^{d}$-valued semimartingale and let $h: \mathbb{R}^{d} \rightarrow \mathbb{R}^{d}$ denote a truncation function, e.g. $h(x)=x 1_{\{|x|\} \leq 1}$.

Definition 1. Let $(B, C, \nu)$ denote a triplet where $B$ is a $\mathbb{R}^{d}$-valued predictable process, $C$ is a predictable process taking values on the space of non-negative symmetric $\mathbb{R}^{d \times d}$ matrices, both with components of finite variation, and $\nu$ is a predictable random measure on $\left(\mathbb{R}_{+} \times \mathbb{R}^{d}\right)$ such that $\left(1 \wedge|x|^{2}\right) * \nu \in \mathcal{V}$. Then $(B, C, \nu)$ is called the characteristics triplet of $X$ if the process

$$
e^{i \lambda^{\top} X}-\int_{0}^{\cdot} e^{i \lambda^{\top} X_{t-}} d K_{t}(i \lambda)
$$

is a local martingale for any $\lambda \in \mathbb{R}^{d}$, where

$$
K_{t}(\zeta)=\zeta^{\top} B_{t}+\frac{1}{2} \zeta^{\top} C_{t} \zeta+\int_{[0, t] \times \mathbb{R}^{d}}\left(e^{\zeta^{\top} x}-1-\zeta^{\top} h(x)\right) \nu(d s, d x)
$$

We refer to $K(\zeta)$ as the cumulant process of $X$ in $\zeta$. 
It can be shown that any semimartingale admits a characteristic triplet which is unique up to a null set. In most applications the triplet $(B, C, \nu)$ is absolutely continuous with respect to the Lebesgue measure, i.e. it holds that

$$
B_{t}=\int_{0}^{t} b_{s} d s, \quad C_{t}=\int_{0}^{t} c_{s} d s, \quad \nu([0, t] \times G)=\int_{0}^{t} F_{s}(G) d s \quad \forall G \in \mathcal{B}^{d},
$$

where $b$ is a $\mathbb{R}^{d}$-valued predictable process, $c$ is a predictable process taking values on the space of non-negative symmetric $\mathbb{R}^{d \times d}$ matrices and $F$ is a transition kernel from $\left(\Omega \times \mathbb{R}_{+}, \mathcal{P}\right)$ to $\left(\mathbb{R}_{+}, \mathcal{B}\right)$ with $F .(\{0\})=0$ and such that $\int\left(1 \wedge|x|^{2}\right) F .(d x)<+\infty$. The cumulant process $K(\zeta)$ is then given by

$$
K_{t}(\zeta)=\int_{0}^{t} \kappa_{s}(\zeta) d s
$$

where

$$
\kappa(\zeta)_{t}=\zeta^{\top} b_{t}+\frac{1}{2} \zeta^{\top} c_{t} \zeta+\int\left(e^{\zeta x}-1-\zeta^{\top} h(x)\right) F_{t}(d x) .
$$

When this is the case, we refer to $(b, c, F)$ as the differential characteristics triplet of $X$ and to $\kappa(\zeta)$ as the Lévy exponent in $\zeta$.

For a semimartingale $X$ with differential triplet $(b, c, F)$ the cumulant process $K(\zeta)$ in (1.43) coincides with the multiplicative compensator of the exponential process $e^{\zeta X}$. More precisely, we have the following result.

Proposition 5. Let $X$ be a $\mathbb{R}^{d}$-valued semimartingale with differential triplet $(b, c, F)$ and let $\zeta \in \mathbb{R}^{d}$ be such that $\int\left(e^{\zeta x}-1-\zeta^{\top} h(x)\right) F .(d x)<\infty$. Then the process

$$
M_{t}(\zeta)=e^{-K_{t}(\zeta)+\zeta X_{t}}
$$

is a local martingale.

Proof. It follows immediately from Kallsen and Shiryaev (2002), Lemma 2.13 and Theorem 2.18.

\subsubsection{Affine Processes}

Affine processes have been introduced by Duffie et al. (2003) where they are defined as a class of time-homogeneous Markov processes with log-characteristic function depending in an affine way on the initial state vector of the process. However, within the semimartingale framework of this work, it is more natural to follow the approach of Kallsen (2006) and 
define an affine process as a $\mathbb{R}^{d}$-valued semimartingale $X$ with differential triplet $(b, c, F)$ given by an affine transformation of $X$ as follows

$$
\begin{aligned}
b_{t} & =\beta_{0}+\sum_{j=1}^{d} \beta_{j} X_{t-}^{j}, \\
c_{t} & =\gamma_{0}+\sum_{j=1}^{d} \gamma_{j} X_{t-}^{j}, \\
F_{t}(B) & =\varphi_{0}(B)+\sum_{j=1}^{d} \varphi_{j}(B) X_{t-}^{j} \quad \forall B \in \mathcal{B}^{d},
\end{aligned}
$$

where $\left(\beta_{j}, \gamma_{j}, \varphi_{j}\right), j=0, \ldots, d$ is a set of $d+1$ Lévy-Khintchine triplets on $\mathbb{R}^{d}$. To ensure that the local covariance matrix $c$ and the local compensator $F$ remain positive, the defining Lévy-Khintchine triplets $\left(\beta_{j}, \gamma_{j}, \varphi_{j}\right), j=0, \ldots, d$ must satisfy the following set of admissibility conditions.

Definition 2 (Kallsen (2006), Definition 3.1). Let $m, n \in \mathbb{N}$ with $m+n=d$, and let $\left(\beta_{j}, \gamma_{j}, \varphi_{j}\right), j=0, \ldots, d$ be a set of $d+1$ Lévy-Khintchine triplets on $\mathbb{R}^{d}$. The triplets $\left(\beta_{j}, \gamma_{j}, \varphi_{j}\right), j=0, \ldots, d$ are called admissible if the following conditions hold

$$
\begin{aligned}
& \beta_{j}^{k}-\int h^{k}(x) \varphi_{j}(d x) \geq 0 \\
& \left.\begin{array}{rl}
\varphi_{j}\left(\left(\mathbb{R}_{+}^{m} \times \mathbb{R}^{n}\right)^{C}\right) & =0 \\
\int h^{k}(x) \varphi(d x) & <0
\end{array}\right\} \quad \text { if } \quad 0 \leq j \leq m, \quad 1 \leq k \leq m, \quad k \neq j ; \\
& \gamma_{j}^{k \ell}=0 \quad \text { if } \quad 0 \leq j \leq m, \quad 1 \leq k, \ell \leq m, \quad \text { unless } k=\ell=j ; \\
& \beta_{j}^{k}=0 \quad \text { if } \quad j \geq m+1, \quad 1 \leq k \leq m \\
& \left.\begin{array}{l}
\gamma_{j}=0 \\
\varphi_{j}=0
\end{array}\right\} \quad \text { if } \quad j \geq m+1
\end{aligned}
$$

The existence of a semimartingale with differential triplet satisfying (1.45) is guaranteed by the following result.

Theorem 1 (Kallsen (2006), Theorem 3.2$)$. Let $\left(\beta_{j}, \gamma_{j}, \varphi_{j}\right), j=0, \ldots, d$ be admissible Lévy-Khintchine triplets and let $\psi_{j}$ denote the corresponding Lévy exponents

$$
\psi_{j}(\zeta)=\zeta^{\top} \beta_{j}+\frac{1}{2} \zeta^{\top} \gamma_{j} \zeta+\int_{\mathbb{R}^{d}}\left(e^{\zeta^{\top} x}-1-\zeta^{\top} h(x)\right) \varphi_{j}(d x)
$$


Assume that the further integrability condition

$$
\int_{\{|x| \geq 1\}}\left|x^{k}\right| \varphi_{j}(d x)<\infty \quad \text { for } 1 \leq j, k \leq m
$$

is satisfied. Then there exists a unique $\mathbb{R}_{+}^{m} \times \mathbb{R}^{n}$-valued semimartingale $X$ with differential characteristic satisfying (1.45). Moreover, it holds that

$$
E\left[e^{\zeta^{\top} X_{s+t}} \mid \mathcal{F}_{s}\right]=\exp \left(A_{t}(\zeta)+B_{t}(\zeta)^{\top} X_{s}\right), \quad \zeta \in \mathbb{C}_{-}^{m} \times i \mathbb{R}^{d-m},
$$

where the functions $A_{t}(\zeta)$ and $B_{t}(\zeta)=\left(B_{t}^{1}(\zeta), \ldots, B_{t}^{d}(\zeta)\right)$ solve the generalized Riccati equations

$$
\begin{aligned}
\frac{d}{d t} A_{t}(\zeta) & =\psi_{0}\left(B_{t}(\zeta)\right) \\
\frac{d}{d t} B_{t}^{j}(\zeta) & =\psi_{j}\left(B_{t}(\zeta)\right) \quad \text { with } B_{0}(\zeta)=\zeta
\end{aligned}
$$

For practical application it is essential to establish whether expression (2) remains valid beyond the domain $\in \mathbb{C}_{-}^{m} \times i \mathbb{R}^{d-m}$ and can be employed for computing exponential moments, when they exist.

Theorem 2 (Duffie et al. (2003), Theorem 2.16). Assume that the functions $A$ and $B$ solving (1.47) and (1.48) have an analytical continuation on an open set $U \subset \mathbb{C}^{d}$ with $0 \in U$. Then for all $\zeta \in U \cap \mathbb{R}^{d}$

$$
E\left[e^{q^{\top} X_{s+t}} \mid \mathcal{F}_{s}\right]<\infty \quad \forall q \in U \cap \mathbb{R}^{d}
$$

and (1.46) holds for all $\zeta \in U$ with $\operatorname{Re} \zeta \in U \cap \mathbb{R}^{d}$.

A classical problem in stochastic analysis is to establish whether local martingales of exponential form are true martingales. A detailed study of exponentially affine, possibly time inhomogeneous martingales, is provided in Kallsen and Muhle-Karbe (2010). Adapting Corollary 3.4 therein to the time-homogeneous case yields the following result

Proposition 6. Let $X=\left(X^{1}, \ldots, X^{d}\right)$ be an $\mathbb{R}_{+}^{m} \times \mathbb{R}^{n}$-valued semimartingale with affine differential characteristics relative to admissible Lévy-Kintchine triplets $\left(\beta_{j}, \gamma_{j}, \varphi_{j}\right), j=$ $0, \ldots, d$. Suppose that for some $1 \leq i \leq d$ the following holds:

$$
\begin{aligned}
& \text { i. } \int_{x_{i}>1} e^{x_{i}} \varphi_{j}(d x)<\infty, \quad j=0, \ldots, m, \\
& \text { ii. } \beta_{j}^{i}+\frac{1}{2} \gamma_{j}^{i i}+\int\left(e^{x_{i}}-1-h_{i}(x)\right) \varphi_{j}(d x)=0, \quad j=0, \ldots, d,
\end{aligned}
$$


iii. $\int_{x_{k}>1} x_{k} e^{x_{i}} \varphi_{j}(d x)<\infty, \quad$ for $j, k=1, \ldots, m$.

Then the process $e^{X^{i}}$ is a martingale. 


\section{Chapter 2}

Closed-form Expansions of the Volatility

Smile in a Mixing Setup 


\title{
Closed-form Expansions of the Volatility Smile in a Mixing Setup
}

\author{
E. Nicolato ${ }^{\dagger}$ and D. Sloth ${ }^{\dagger}$
}

$\dagger$ Department of Economics and Business, Aarhus University, Aarhus

\begin{abstract}
We derive a closed-form expansion of implied volatility and at-the-money skew. The expansion is simple, transparent, and easy to implement. It is based on a mixing representation of the underlying model. We apply the expansion to the Heston stochastic volatility model as well as the Variance Gamma model and provide a clear analysis of the at-the-money term structure of implied volatility. However, the expansion may be used for a broad class of option pricing models displaying both jumps and stochastic volatility in the underlying dynamics. Finally, we explore two domains of application of the expansion. One as a control variate to speed up Fourier option pricing, and another one as a fast, first-order approach for calibration to at-the-money volatilities and skews.
\end{abstract}

\section{$2.1 \quad$ Introduction}

Take it hard to the hoop! This basketball expression beautifully captures what analytical approximations are all about. They are rough - this is what makes them approximations - but they often constitute the most lucid and direct approach. In quantitative finance, good approximations help model handling by speeding up calibration to market-observed quantities and, even more importantly, they enhance our understanding of the model's analytical features. Numerical methods for option pricing are extremely powerful in terms of accuracy. Nevertheless, they are hermetically closed; they fail to establish a clear link between the structural properties of the model and its effects on the characteristics of the 
generated volatility surface. For these reasons, analytical approximations have been and continue to be proposed in the literature.

In the context of stochastic volatility models, Lewis (2000) derives an asymptotic expansion of implied volatility assuming small volatility of volatility. Corresponding results are obtained by Lee (2001) assuming slow mean-reversion of volatility, while Fouque et al. (2000) study the case of fast mean-reverting volatility. Hagan et al. (2002) derive a smalltime expansion in the SABR model using singular pertubation techniques. Medvedev and Scaillet (2007) develop an asymptotic expansion in a jump-diffusion stochastic volatility model. Small-time asymptotics of implied volatility in the Heston model are analyzed in Forde et al. (2012). Similar work can be found in Berestycki et al. (2004), Osajima (2007) and Forde (2008), whereas Benhamou et al. (2009) and Larsson (2012) suggest implied volatility expansions based on Malliavin calculus. Common to the proposed expansions is that they are only valid for either short or long maturities.

In this paper we develop a closed-form expansion of implied volatility and at-the-money variance skew. The expansion is simple, transparent, and easy to implement. Moreover, in contrast to the majority of the previous literature, the purpose of this work is not obtain an asymptotic expansion valid only for extreme maturities or strikes. Instead, our aim is to develop a general implied volatility expansion useful in the large region of the volatility surface where options are actually traded. In this respect, the present work is most closely related to, at least in spirit, the most-likely-path approximation of Gatheral (2006), the singular expansion of Jäckel (2009) as well as the recent works of Drimus (2011), Poulsen and Ribeiro (2012).

This paper extends the work outlined in Chapter 1. Most noticeably, the model framework has been generalized to include classical stochastic volatility models beyond the pure-jump type models investigated in the previous chapter. Moreover, in this paper the expansion is derived directly in implied volatility space which provides an explicit link between the characteristics of the option pricing model and the shape of the implied volatility surface. Also, while the expansion can be used for a broad class of option pricing models, we concentrate in this paper on a few models and study the asymptotic behavior of the at-the-money term structure of implied volatility and volatility skew. Specifically, we illustrate the use of the implied volatility expansion for the popular Heston (1993) stochastic volatility model and the Variance Gamma model of Madan and Seneta (1990).

The neat simplicity of the expansion and its broad applicability across various models is due to the fact that it is based on 'summary statistics' such as moments and cross- 
moments of the underlying model components. Moreover, the expansion brings about the key insight that implied volatility can be understood as an expected volatility adjusted for risks of movements in the underlying asset price or its volatility. The risk correction terms are governed by higher-order option risks - yet, their magnitudes are purely determined by the characteristics of the model. This way of interpreting the volatility smile is consistent with industry practice. Market participants cope with smile effects by hedging not only classical risks, like Delta risk, but also higher-order risks such as convexity in the volatility and dependence of the option's Delta on the level of volatility.

The decomposition of the volatility smile clarifies how the analytical features of the underlying model are translated into implied volatilities. This is not to be underestimated since understanding how a model responds to moves of its parameters or underlying quantities is crucial for its introduction and use in market trading. Furthermore, the decomposition allows for systematic comparison of complex option pricing models by quantifying how option risks affect the volatility smile across different models. For example, in a simplified model assuming zero correlation between the stock price and its volatility, risks associated with movements in the stock price do not affect implied volatility, whereas these risks will have a nonzero effect in models allowing for correlation.

We explore two domains of application of the expansion. First, we propose an expansionbased control variate for option pricing based on Fourier transform methods to improve convergence properties of the embedded numerical integration. Andersen and Andreasen (2002) suggest using the Black-Scholes price as a control. This, unfortunately, constitutes a Catch-22 as to which volatility one should use in the Black-Scholes pricing formula. In practice, the choice has usually been more or less arbitrary. We argue that the expansion of implied volatility gives a consistent choice of the input volatility. Our numerical results confirm that this approach brings about a significant speed-up reducing the number of required function evaluations by more than half. Secondly, we propose a fast, first-order model calibration approach. Specifically, we suggest using the expansion to jointly calibrate to at-the-money volatilities and skews. Besides a gain in computational speed, the advantage of this approach is that it requires fewer data points compared to the usual procedure of calibrating to the whole surface of option prices.

The paper is organized as follows. In Section 2.2 we outline the model setup. In Section 2.3 we develop the main theoretical results underlying the expansion. In Section 2.4 we study the numerical accuracy of the expansion and its analytical properties under different model dynamics. In Section 2.5 we study two different domains of application. 
Finally, in Section 2.6 we make some concluding remarks.

\subsection{The Mixing Setup}

In this work we consider a general modeling framework which extends classical stochastic volatility models to allow for jumps and yet it conveniently retains the conditional lognormality of asset returns. Specifically, we assume that under the pricing measure $\mathbb{Q}$, the price $S$ of an underlying asset evolves according to the dynamics

$$
S_{t}=S_{t}^{e f f} \exp \left(-\frac{1}{2} V_{t}^{e f f}+W_{V_{t}^{e f f}}\right), \quad V_{0}^{e f f}=0, S_{0}^{e f f}=S_{0}
$$

where the process $S^{e f f}$ is a positive martingale starting at the spot value $S_{0}$ of the underlying, $V^{e f f}$ is an increasing process, and $W$ is a standard Brownian motion independent of the pair $\left(S^{e f f}, V^{e f f}\right)$ which is evaluated at the random time $V^{e f f}$. Given the independence between $W$ and ( $\left.S^{e f f}, V^{e f f}\right)$, the process $S$ is a martingale. This means (2.1) describes the risk-neutral dynamics of the asset price process in a simplified economy with zero interest rates and dividend yields. Allowing for positive rates is a straightforward extension.

This model construction is, in fact, just a generalization of the seminal Black-Scholes model

$$
S_{t}=S_{0} \exp \left(-\frac{1}{2} \sigma^{2} t+W_{\sigma^{2} t}\right), \quad \sigma>0
$$

obtained by randomizing the spot value $S_{0}$ and the deterministic total variance $\sigma^{2} t$ via their natural stochastic counterparts, i.e., the martingale $S^{\text {eff }}$ and the increasing process $V^{e f f}$. Hence, adopting the terminology of Lewis (2000), the processes $S^{\text {eff }}$ and $V^{e f f}$ are called the effective spot and the effective variance respectively, while we refer to the model framework (2.1) as the mixing setup.

Application of this model class depends on the tractability of what we term the effective Laplace transform. This is the joint Laplace transform of the effective log-price $X_{t}^{e f f}=$ $\log S_{t}^{\text {eff }}$ and the effective variance $V_{t}^{\text {eff }}$

$$
\mathcal{L}_{t}^{e f f}(u, w)=\mathbb{E}\left[e^{u X_{t}^{e f f}+w V_{t}^{e f f}}\right]
$$

defined in the domain $\mathcal{D}_{t}=\left\{(u, w) \in \mathbb{C}^{2}: \mathbb{E}\left[\left|e^{u X_{t}^{e f f}}+w V_{t}^{e f f}\right|\right]<+\infty\right\}$. For example, option prices can be computed by Fourier or other transform methods whenever the effective 
Laplace transform is available in closed form as the characteristic function of the logprice $X_{T}=\log S_{T}$ is given by

$$
\mathbb{E}\left[e^{i u X_{t}}\right]=\mathcal{L}_{t}^{e f f}\left(i u,-\frac{1}{2}\left(i u+u^{2}\right)\right)
$$

In the sequel, the effective Laplace transform $\mathcal{L}_{t}^{\text {eff }}$ plays an essential part when computing the terms of the implied volatility expansion that we develop.

The mixing setup embeds a variety of popular option pricing models. Noticeably, at one end of the spectrum, it contains stochastic volatility models of the following type

$$
\begin{aligned}
& d S_{t}=S_{t} \sqrt{v_{t}}\left(\sqrt{1-\rho^{2}} d W+\rho d B\right) \\
& d v_{t}=\mu\left(v_{t}, t\right) d t+\sigma\left(v_{t}, t\right) d B_{t}, \quad v_{0}>0
\end{aligned}
$$

where $W$ and $B$ are independent standard Brownian motions, $-1 \leqslant \rho \leqslant 1$ is the correlation parameter, and the drift $\mu$ and the diffusion $\sigma \geqslant 0$ coefficients satisfy the usual regularity conditions to guarantee that the instantaneous variance process $v$ remains non-negative and enjoys enough integrability. The mixing representation (2.1) follows immediately by setting

$$
\begin{aligned}
V_{t}^{e f f} & =\left(1-\rho^{2}\right) \int_{0}^{t} v_{s} d s, \\
S_{t}^{e f f} & =S_{0} \exp \left(-\frac{\rho^{2}}{2} \int_{0}^{t} v_{s} d s+\rho \int_{0}^{t} \sqrt{v_{s}} d B_{s}\right),
\end{aligned}
$$

and from the Dambis-Dubins-Schwarz representation theorem for continuous local martingales (see, e.g., Revuz and Yor (1999)). In this case, the effective Laplace transform $\mathcal{L}_{t}^{e f f}(u, w)$ defined in (2.2) can be reformulated in the following manner

$$
\mathcal{L}_{t}^{e f f}(u, w)=\mathbb{E}\left[e^{u X_{t}+\left(1-\rho^{2}\right)\left(w+\frac{1}{2} u-\frac{1}{2} u^{2}\right) \int_{0}^{t} v_{s} d s}\right]=\mathcal{L}_{t}^{X V}\left(u,\left(1-\rho^{2}\right)\left(w+\frac{1}{2} u-\frac{1}{2} u^{2}\right)\right),
$$

where $\mathcal{L}_{t}^{X V}$ denotes the joint Laplace transform of the log-price $X_{t}$ and its integrated variance $V_{t}=\int_{0}^{t} v_{s} d s$. Hence, $\mathcal{L}_{t}^{e f f}$ can be easily computed for all those stochastic volatility models for which $\mathcal{L}_{t}^{X V}$ is available in closed-form. This includes stochastic volatility models such as, for instance, the Hull and White (1987) model, the Stein and Stein (1991) model, the Heston (1993) model, and the 3/2 model popularized in Lewis (2000).

At the other end of the model spectrum, one finds option pricing models where the asset price $S$ does not display any diffusion component but evolves uniquely due to the presence of jumps. Within the mixing setup, models of this kind are obtained by specifying 
the effective variance $V^{e f f}$ as a purely jumping semimartingale without drift and cumulant $\operatorname{process}^{1} K^{V}(\zeta)$ given by

$$
K_{t}(\zeta)=\int_{0}^{t} \kappa_{s}(\zeta) d s \quad \text { with } \quad \kappa_{t}(\zeta)=\int_{\mathbb{R}_{+}}\left(e^{\zeta x}-1\right) F_{t}(d x), \quad \zeta \in \mathbb{C},
$$

where $F$ is the differential compensator of the random measure of jumps, i.e., a transition kernel from $\left(\Omega \times \mathbb{R}_{+}, \mathcal{P}\right)$ to $\left(\mathbb{R}_{+}, \mathcal{B}\right)$ with $F .(\{0\})=0$ and $\int_{\mathbb{R}_{+}}(1 \wedge x) F .(d x)<+\infty$. To complete the description, we construct the effective spot by scaling and exponentially compensating the effective variance as follows

$$
S^{e f f}=S_{0} \exp \left(-K_{t}(c)+c V_{t}^{e f f}\right)
$$

where $c$ is a real parameter for which $K(c)$ is well defined. The parameter $c$ allows for correlation between the price process $S$ and its effective variance $V^{e f f}$, and at the same time it governs the skewness in the asset-returns distributions. Therefore, we will refer to it as the skewness parameter.

This modeling approach for purely jumping option pricing models was introduced in Nicolato and Sloth (2013) where several concrete specifications for $V^{\text {eff }}$ are considered in details. For example, exponential Lévy models are obtained by modeling the effective variance as a drift-less Lévy subordinator $Z$ with Lévy measure $\nu(d x)$ and deterministic cumulant process $K_{t}(\zeta)=t \kappa(\zeta)$ where $\kappa$ is the Lévy exponent

$$
\kappa(\zeta)=\int_{\mathbb{R}_{+}}\left(e^{\zeta x}-1\right) \nu(d x) .
$$

If the skewness parameter $c$ is chosen so that $\kappa(c)<+\infty$, the mixing representation $(2.1)$ of the exponential Lévy price $S$ is obtained by setting

$$
\begin{aligned}
V^{e f f} & =Z_{t} \\
S^{e f f} & =S_{0} \exp \left(-t \kappa(c)+c Z_{t}\right) .
\end{aligned}
$$

In this case the effective Laplace transform has the following simple expression

$$
\mathcal{L}_{t}^{e f f}(u, w)=S_{0}^{u} e^{t(\kappa(u c+w)-u \kappa(c))},
$$

and it is available in closed-form whenever the Lévy exponent $\kappa$ can be computed explicitly. Popular exponential Lévy models that can constructed as outlined include the

\footnotetext{
${ }^{1}$ For a detailed study of semimartingale characteristics and cumulant processes see, e.g., Kallsen and Shiryaev (2002).
} 
Variance Gamma model introduced by Madan and Seneta (1990), the Hyperbolic models examined by Eberlein and Keller (1995), the Normal Inverse Gaussian model of BarndorffNielsen (1998) as well as the CGMY model proposed by Carr et al. (2002).

In addition, we obtain purely jumping models belonging to the Lévy stochastic volatility class developed in Carr et al. (2003) by specifying the effective variance process $V^{e f f}$ as a drift-less subordinator $Z$ evaluated at an absolutely continuous stochastic time $V$

$$
V_{t}^{e f f}=Z_{V_{t}} \quad \text { with } \quad V_{t}=\int_{0}^{t} v_{s} d s
$$

where the instantaneous activity rate $v$ is given by some positive process independent of $Z$. The differential compensator $F$ and the cumulant process $K$ of $V^{\text {eff }}$ are then given by

$$
F_{t}(d x)=\nu(d x) y_{t} \quad \text { and } \quad K_{t}(\zeta)=\kappa(\zeta) V_{t},
$$

where $\nu$ and $\kappa$ denote the Lévy measure and the Lévy exponent of $Z$. Once again, if the skewness parameter $c$ is chosen within the domain of $\kappa$, we achieve the mixing representation (2.1) by specifying

$$
\begin{aligned}
V_{t}^{\text {eff }} & =Z_{V_{t}} \\
S^{\text {eff }} & =S_{0} \exp \left(-t \kappa(c) V_{t}+c Z_{V_{t}}\right) .
\end{aligned}
$$

The corresponding effective Laplace transform can be expressed as follows

$$
\mathcal{L}_{t}^{e f f}(u, w)=S_{0}^{u} \mathbb{E}\left[e^{V_{t}(\kappa(u c+w)-u \kappa(c))}\right]=S_{0}^{u} \mathcal{L}_{t}^{V}(\kappa(u c+w)-u \kappa(c))
$$

and can be computed explicitly whenever the the Laplace transform $\mathcal{L}_{t}^{V}$ of the stochastic clock and the Levy exponent $\kappa(\zeta)$ of the subordinator $Z$ are available in closed-form.

\subsection{Implied Volatility Expansions}

In this section we develop the implied volatility expansion for the mixing setup introduced above. A key feature of this setup is the fact that conditionally on each realization of $\left(S_{t}^{e f f}, V_{t}^{\text {eff }}\right)$, the asset price $S_{t}$ given by $(2.1)$ is log-normally distributed. Similar to Hull and White (1987) and Romano and Touzi (1997), it follows that the price $C\left(S_{0}, K, \tau\right)$ of a European call option with strike $K$ and expiry $\tau>0$ admits the following mixing representation

$$
C\left(S_{0}, K, \tau\right)=\mathbb{E}\left[\left(S_{\tau}-K\right)^{+}\right]=\mathbb{E}\left[C_{B S}\left(S_{\tau}^{e f f}, V_{\tau}^{e f f}\right)\right]
$$


where $C_{B S}(S, V)$ denotes the Black-Scholes price of a call option in terms of the spot price and total variance of the underlying asset. That is,

$$
C_{B S}(S, V)=S \Phi\left(d_{+}\right)-K \Phi\left(d_{-}\right), \quad \text { where } \quad d_{ \pm}=-\frac{x}{\sqrt{V}} \pm \frac{\sqrt{V}}{2},
$$

with $x=\log (K / S)$ denoting the log-moneyness.

Generalizing a technique introduced by Lewis (2000), we consider two different expansions of the true call option price $C\left(S_{0}, K, T\right)$. First, we apply a two-dimensional Taylor series expansion of the mixing representation (2.14) around the point $\left(S_{0}, \mathbb{E} V_{\tau}^{\text {eff }}\right)$. This yields the following $\langle S, V\rangle$-expansion

$$
\begin{aligned}
C\left(S_{0}, K, \tau\right)= & C_{B S}\left(S_{0}, \mathbb{E} V_{\tau}^{\text {eff }}\right)+\left.\frac{1}{2 !} \mathbb{E}\left[\left(S_{\tau}^{e f f}-S_{0}\right)^{2}\right] \frac{\partial^{2} C_{B S} C}{\partial S^{2}}\right|_{\left(S_{0}, \mathbb{E} V_{\tau}^{e f f}\right)} \\
& +\left.\frac{1}{2 !} \mathbb{E}\left[\left(V_{\tau}^{e f f}-\mathbb{E} V_{\tau}^{e f f}\right)^{2}\right] \frac{\partial^{2} C_{B S}}{\partial V^{2}}\right|_{\left(S_{0}, \mathbb{E} V_{\tau}^{e f f}\right)} \\
& +\left.\mathbb{E}\left[\left(S_{\tau}^{e f f}-S_{0}\right)\left(V_{\tau}^{e f f}-\mathbb{E} V_{\tau}^{e f f}\right)\right] \frac{\partial^{2} C_{B S}}{\partial S \partial V}\right|_{\left(S_{0}, \mathbb{E} V_{\tau}^{e f f}\right)}+\cdots
\end{aligned}
$$

where we have used the fact that $\mathbb{E} S_{\tau}^{e f f}=S_{0}$.

Next, we note that by definition the call price $C\left(S_{0}, K, \tau\right)$ is equal to the Black-Scholes price for the same option characteristics evaluated at the implied volatility $I=I\left(S_{0}, K, \tau\right)$. If we denote the implied total variance by $\Sigma_{\tau}=\tau I^{2}$, we have that

$$
C\left(S_{0}, K, \tau\right) \equiv C_{B S}\left(S_{0}, \Sigma_{\tau}\right)
$$

We then expand this expression in the second variable $\Sigma$ around the expected effective variance $\mathbb{E} V_{\tau}^{\text {eff }}$ using a one-dimensional Taylor series. This step yields the second expansion, termed the $\langle\Sigma\rangle$-expansion, as follows

$$
\begin{aligned}
C\left(S_{0}, K, \tau\right)= & C_{B S}\left(S_{0}, \mathbb{E} V_{\tau}^{e f f}\right)+\left.\left(\Sigma_{\tau}-\mathbb{E} V_{\tau}^{e f f}\right) \frac{\partial C_{B S}}{\partial V}\right|_{\left(S_{0}, \mathbb{E} V_{\tau}^{e f f}\right)} \\
& +\left.\frac{1}{2 !}\left(\Sigma_{\tau}-\mathbb{E} V_{\tau}^{e f f}\right)^{2} \frac{\partial^{2} C_{B S}}{\partial V^{2}}\right|_{\left(S_{0}, \mathbb{E} V_{\tau}^{e f f}\right)}+\cdots
\end{aligned}
$$

Equating the right-hand sides of (2.15) and (2.16) and solving for $\Sigma_{\tau}$ yields a formal expansion of the total implied variance.

If we truncate the $\langle\Sigma\rangle$-expansion (2.16) to the first order and the $\langle S, V\rangle$-expansion (2.15) to the $q$-th order, we obtain the following simple polynomial expansion 


$$
\Sigma_{\tau} \approx \mathbb{E} V_{\tau}^{e f f}+\sum_{k=2}^{q} \sum_{l=0}^{k} \frac{\mathfrak{D}_{s^{l} v^{k-l}}}{l !(k-l) !} \mathbb{E}\left[\left(S_{\tau}^{e f f}-S_{0}\right)^{l}\left(V_{\tau}^{e f f}-\mathbb{E} V_{\tau}^{e f f}\right)^{k-l}\right],
$$

where we have employed the following compact notation

$$
\mathfrak{D}_{s^{m} v^{n}} \equiv\left(\frac{\partial C_{B S}}{\partial V}\right)^{-1} \frac{\partial^{m+n} C_{B S}}{\partial S^{m} \partial V^{n}}, \quad n, m \geqslant 0,
$$

for the Vega-normalized Black-Scholes derivatives. In the sequel, we will refer to these derivatives as normalized Greeks and to expression (2.17) as the $\mathcal{O}(q)$-order approximation. Finiteness of the moments $\mathbb{E}\left[\left(V_{t}^{\text {eff }}\right)^{m}\left(S_{t}^{\text {eff }}\right)^{n}\right]$ entering the expansion depends on the regularity and tractability of the effective Laplace transform $\mathcal{L}_{t}^{\text {eff }}$ defined in (2.2). Specifically, if the pair $(0, n)$ lies in the interior domain $\dot{\mathcal{D}}_{t}$ of $\mathcal{L}_{t}^{\text {eff }}$, then we have that $\mathbb{E}\left[\left(V_{t}^{\text {eff }}\right)^{m}\left(S_{t}^{\text {eff }}\right)^{n}\right]<\infty$ and the moments can be computed by the following differentiation

$$
\mathbb{E}\left[\left(V_{t}^{\text {eff }}\right)^{m}\left(S_{t}^{e f f}\right)^{n}\right]=\left.\frac{\partial^{m} \mathcal{L}_{t}^{e f f}(u, w)}{\partial u^{m}}\right|_{u=0, w=n},
$$

see, e.g., Hoffmann-Jørgensen (1994), Section 4.19.

\subsubsection{A Simple Quadratic Approximation}

The second order expansion is of particular interest as it yields a simple approximation of the implied instantaneous variance $I^{2}(x, \tau)$ which is quadratic in the log-moneyness $x=\log K / S$. Moreover, it extends the results previously obtained by Ball and Roma (1994) and Lewis (2000) in the special case of stochastic volatility models restricted to zero correlation between the asset price and its volatility. We obtain the following

$$
I^{2}(x, \tau) \approx I_{Q}^{2}(x, \tau)=\frac{\mathbb{E} V_{\tau}^{e f f}}{\tau}+\frac{\mathbb{V a r}\left[S_{\tau}^{e f f}\right]}{2 \tau} \mathfrak{D}_{s s}+\frac{\mathbb{V a r}\left[V_{\tau}^{e f f}\right]}{2 \tau} \mathfrak{D}_{v v}+\frac{\mathbb{C} o v\left[S_{\tau}^{e f f}, V_{\tau}^{e f f}\right]}{\tau} \mathfrak{D}_{s v},
$$

where the normalized Gamma $\mathfrak{D}_{s s}$, Volga $\mathfrak{D}_{v v}$ and Vanna $\mathfrak{D}_{s v}$ are given by

$$
\mathfrak{D}_{s s}=\frac{2}{S^{2}}, \quad \mathfrak{D}_{v v}=\frac{x^{2}}{2 V^{2}}-\frac{1}{8}-\frac{1}{2 V}, \quad \mathfrak{D}_{s v}=\frac{x}{S V}-\frac{1}{2 S} .
$$

The approximation (2.19) provides a neat interpretation of how the implied variance is generated by correcting the rough effective estimate $\mathbb{E} V_{\tau}^{\text {eff }} / \tau$ with a series of adjustments accounting for higher-order option risks measured per unit of Vega risk. While the normalized Gamma risk only contributes to the level of volatility in the approximation, the 
normalized Vanna term determines the slope of the smile, while the Volga term introduces convexity in the surface. Re-arranging the terms in (2.19) yields the following expression

$$
I_{Q}^{2}(x, \tau)=\mathfrak{I}_{0}(\tau)+\mathfrak{I}_{1}(\tau) x+\mathfrak{I}_{2}(\tau) x^{2},
$$

where the functionals $\mathfrak{I} .(\tau)$ describing the at-the-money term structure are given by

$$
\begin{aligned}
& \mathfrak{I}_{0}(\tau)=\frac{\mathbb{E} V_{\tau}^{\text {eff }}}{\tau}+\frac{\mathbb{V} a r\left[S_{\tau}^{\text {eff }}\right]}{S_{0}^{2} \tau}+\frac{\mathbb{C o v}\left[S_{\tau}^{e f f}, V_{\tau}^{\text {eff }}\right]}{2 S_{0} \tau}-\frac{\mathbb{V} a r\left[V_{\tau}^{e f f}\right]}{4 \tau\left(\mathbb{E} V_{\tau}^{e f f}\right)^{2}}\left(\mathbb{E} V_{\tau}^{e f f}+\frac{1}{4}\left(\mathbb{E} V_{\tau}^{e f f}\right)^{2}\right), \\
& \mathfrak{I}_{1}(\tau)=\frac{\mathbb{C o v}\left[S_{\tau}^{e f f}, V_{\tau}^{e f f}\right]}{S_{0} \mathbb{E} V_{\tau}^{e f f}} \frac{1}{\tau}, \\
& \mathfrak{I}_{2}(\tau)=\frac{\mathbb{V a r}\left[V_{\tau}^{\text {eff }}\right]}{4\left(\mathbb{E} V_{\tau}^{\text {eff }}\right)^{2}} \frac{1}{\tau} .
\end{aligned}
$$

$\mathfrak{I}_{0}(\tau)$ approximates the at-the-money level of the implied variance as a function of timeto-expiry $\tau$

$$
I^{2}(0, \tau) \approx \mathfrak{I}_{0}(\tau)
$$

We observe that the level is composed of a natural leading term, the annualized average effective variance, plus some corrections accounting for randomness both in the effective spot and variance. The at-the-money skewness of the implied variance is approximated by

$$
\left.\frac{\partial I^{2}}{\partial x}\right|_{x=0} \approx \mathfrak{I}_{1}(\tau)
$$

The covariance between the effective variance and the effective spot dictates the sign of the at-the-money skew, producing a symmetric smile in the uncorrelated case. As the effective spot $S^{\text {eff }}$ is a martingale by definition, we can express the covariance as follows

$$
\mathbb{C o v}\left[S_{\tau}^{e f f}, V_{\tau}^{e f f}\right]=S_{0}\left(\mathbb{E}^{\mathbb{Q}^{1}} V_{\tau}^{e f f}-\mathbb{E} V_{\tau}^{e f f}\right),
$$

where $\mathbb{E}^{\mathbb{Q}^{1}}$ denotes the expectation taken under the probability measure defined by $d \mathbb{Q}^{1} / d \mathbb{Q}=$ $S^{e f f} / S_{0}$. This observation allows us to relate the at-the-money skew to the correlation parameter $\rho$ in the classical stochastic volatility models or to the skewness parameter $c$ in the case of purely jumping asset models.

First, let us consider the stochastic volatility model described by $(2.3)$ for which $\mathbb{Q}^{1}$ is defined by the Radon-Nikodym derivative

$$
\left.\frac{d \mathbb{Q}^{1}}{d \mathbb{Q}}\right|_{\mathcal{F}_{t}}=\mathcal{E}\left(\rho \int_{0}^{t} \sqrt{v_{s}} d B_{s}\right)=\exp \left(-\frac{\rho^{2}}{2} \int_{0}^{t} v_{s} d s+\rho \int_{0}^{t} \sqrt{v_{s}} d B_{s}\right) .
$$


By Girsanov's theorem, the $\mathbb{Q}^{1}$-dynamics of the instantaneous variance process $v$ are given by

$$
d v_{t}=\left(\mu\left(v_{t}, t\right)+\rho \sqrt{v_{t}} \sigma\left(v_{t}, t\right)\right) d t+\sigma\left(v_{t}, t\right) d B_{t}^{1},
$$

where $B_{t}^{1}=B_{t}-\rho \int_{0}^{t} \sqrt{v_{s}} d s$ is a $\mathbb{Q}^{1}$-Brownian motion. Hence, under sufficient integrability conditions, which are assumed satisfied, we have

$$
\mathbb{E}^{\mathbb{Q}^{1}} v_{t}=v_{0} t+\mathbb{E}\left[\int_{0}^{t}\left(\mu\left(v_{s}, s\right)+\rho \sqrt{v_{s}} \sigma\left(v_{s}, s\right)\right) d s\right],
$$

and since $\sigma>0$, we conclude that $\mathbb{E}^{\mathbb{Q}^{1}}\left[\int_{0}^{\tau} v_{s} d s\right] \gtrless \mathbb{E}\left[\int_{0}^{\tau} v_{s} d s\right]$ when $\rho \gtrless 0$. Therefore, the quadratic approximation (2.20) explains the well known fact that stochastic volatility models display a positive or negative at-the-money volatility skew in correspondence to a positive or negative correlation parameter $\rho$.

We can proceed in a similar manner for purely jumping asset models described by (2.7) for which $\mathbb{Q}^{1}$ is defined by the generalized Esscher transform

$$
\left.\frac{d \mathbb{Q}^{1}}{d \mathbb{Q}}\right|_{\mathcal{F}_{t}}=\exp \left(-K_{t}(c)+c V_{t}^{e f f}\right) .
$$

where $c$ is the skewness parameter. A Girsanov-type theorem for semimartingales (see e.g. Jacod and Shiryaev (2003), III.3.24) shows that under $\mathbb{Q}^{1}$ the effective variance $V^{\text {eff }}$ is still driftless with differential compensator of the random measure of jumps given by

$$
F_{t}^{\mathbb{Q}^{1}}(d x)=e^{c x} F_{t}(d x)
$$

implying that

$$
\mathbb{E}^{\mathbb{Q}^{1}} V_{\tau}^{e f f}-\mathbb{E} V_{\tau}^{e f f}=\mathbb{E}\left[\int_{0}^{\tau} x\left(e^{c x}-1\right) F_{s}(d x) d s\right]
$$

Once again, the approximated at-the-money skew is consistent with the behavior of the true model, yielding a positive or negative skew in correspondence to a positive or negative sign of the skewness parameter $c$.

Finally, the implied variance curvature or convexity is approximated by

$$
\frac{\partial^{2} I^{2}}{\partial x^{2}} \approx 2 \mathfrak{I}_{2}(\tau)
$$

The term structure of the curvature term reflects a more pronounced shape for high values of variance-of-variance, in accordance with typical patterns displayed by stochastic volatility models as well as purely jumping asset models. 


\subsection{Analytics and Numerics for Benchmark Models}

In this section we examine the implied volatility expansion (2.17) for two popular option pricing models. We study both the numerical accuracy of the expansion and its analytical properties under the different model dynamics, mainly focusing on the quadratic approximation (2.20) from the previous section.

\subsubsection{Heston Dynamics}

The most widely known representative of the stochastic volatility model class (2.3) is the Heston (1993) model. The instantaneous variance is described by the square-root process of Cox et al. (1985)

$$
d v_{t}=\kappa\left(\eta-v_{t}\right) d t+\lambda \sqrt{v_{t}} d B_{t}, \quad v_{0}>0
$$

where $\kappa, \eta$ and $\lambda$ are positive parameters representing the speed of mean reversion, the long-term level, and the so-called volatility-of-volatility parameter (for short, vol-of-vol). The joint Laplace transform of the log-price and its integrated variance is given by

$$
\mathcal{L}_{t}^{X V}(u, w)=\mathbb{E}\left[e^{u X_{t}+w \int_{0}^{t} v_{s} d s}\right]=S_{0}^{u} A(u, w, t) e^{B(u, w, t) v_{0}}
$$

where

$$
\begin{aligned}
A(u, w, T) & =\left(\frac{e^{\widehat{\kappa} t / 2}}{\cosh (\varpi t / 2)+\widehat{\kappa} / \varpi \sinh (\varpi t / 2)}\right)^{2 \widehat{\kappa} \eta / \lambda^{2}} \\
B(u, w, T) & =\left(\frac{2 w-u+u^{2}}{\widehat{\kappa}+\varpi \operatorname{coth}(\varpi t / 2)}\right)
\end{aligned}
$$

with

$$
\widehat{\kappa}=\kappa-u \rho \lambda, \quad \widehat{\eta}=\eta \frac{\kappa}{\kappa-u \rho \lambda}, \quad \varpi=\sqrt{\widehat{\kappa}^{2}-2 \lambda^{2}\left(w-u / 2+u^{2} / 2\right)} .
$$

Thus, the effective joint Laplace transform $\mathcal{L}_{t}^{\text {eff }}$ defined in (2.2) can be computed analytically via (2.5) with differentiation (2.18) providing the terms of the expansion in closed-form. The explicit moments entering the quadratic approximation are given as 
follows

$$
\begin{aligned}
\mathbb{E}\left[V_{\tau}^{e f f}\right] & =\left(1-\rho^{2}\right)\left(\tau \eta+\frac{1-e^{-\tau \kappa}}{\kappa}\left(v_{0}-\eta\right)\right), \\
\operatorname{Cov}\left[S_{\tau}^{e f f}, V_{\tau}^{e f f}\right] & =S_{0}\left(1-\rho^{2}\right)\left(\tau \bar{\eta}+\frac{1-e^{-\tau \bar{\kappa}}}{\bar{\kappa}}\left(v_{0}-\bar{\eta}\right)\right)-S_{0} \mathbb{E}\left[V_{\tau}^{e f f}\right], \\
\operatorname{Var}\left[S_{\tau}^{e f f}\right] & =\mathcal{L}_{\tau}^{X V}\left(2, \rho^{2}-1\right)-S_{0}^{2},
\end{aligned}
$$

where

$$
\bar{\eta}=\frac{\kappa \eta}{\kappa-\rho \lambda} \quad \text { and } \quad \bar{\kappa}=\kappa-\rho \lambda .
$$

Finally, the variance of the effective variance is given by

$$
\begin{aligned}
\operatorname{Var}\left[V_{\tau}^{e f f}\right]=\left(1-\rho^{2}\right)^{2} & \left(\frac{v_{0} \lambda^{2}}{\kappa^{3}}-\frac{5 \eta \lambda^{2}}{2 \kappa^{3}}+\frac{2 \eta \lambda^{2}}{\kappa^{3}} e^{-\tau \kappa}+\frac{\left(\eta-2 v_{0}\right) \lambda^{2}}{2 \kappa^{3}} e^{-2 \tau \kappa}\right. \\
& \left.+\frac{\eta \lambda^{2}}{\kappa^{2}} \tau+\frac{2\left(\eta-v_{0}\right) \lambda^{2}}{\kappa^{2}} \tau e^{-\tau \kappa}\right) .
\end{aligned}
$$

The terms of higher-order approximations are computed along the same lines. Figure 2.1 plots the implied volatilities and their approximations for several times to maturity $\tau=5,1,1 / 2,1 / 4$. We use the parameters $\kappa=1.15, \eta=0.04, \lambda=0.2, v_{0}=0.04$, and $\rho=-0.4$ from Forde et al. (2012). The true implied volatilities are computed based on the Fourier transform approach of Lewis (2001) and Lipton (2002). One observes from Figure 2.1 that the approximations are fairly accurate across any reasonable strike range. For example, the mean absolute error is $7 \mathrm{bps}$ for 3 -months options and only 5 bps for 6 -month options across a relative strike range of $[0.8 ; 1.2]$. In addition, the plots visualize how the quality of the approximation increases in the strike dimension as time to maturity increases. Furthermore, the quadratic approximation is generally superior around at-the-money compared to the higher-order approximations. In fact, there is generally no guarantee that higher-orders of our expansion will result in more accurate approximations. Carr (2000) shows Taylor expansions of Black-Scholes prices in $S$ and $V$ have a finite radius of convergence while to obtain the $\langle\xi, V\rangle$-expansion and $\langle\Sigma\rangle$-expansion Black-Scholes prices are expanded over the entire domain.

If we substitute the moments (2.27)-(2.30) into expressions (2.21) and (2.22), we obtain the corresponding closed-form approximations of the at-the-money implied variance $\mathfrak{I}_{0}(\tau)$ and at-the-money skew $\mathfrak{I}_{1}(\tau)$. Simple calculations show that as the maturity shrinks to 


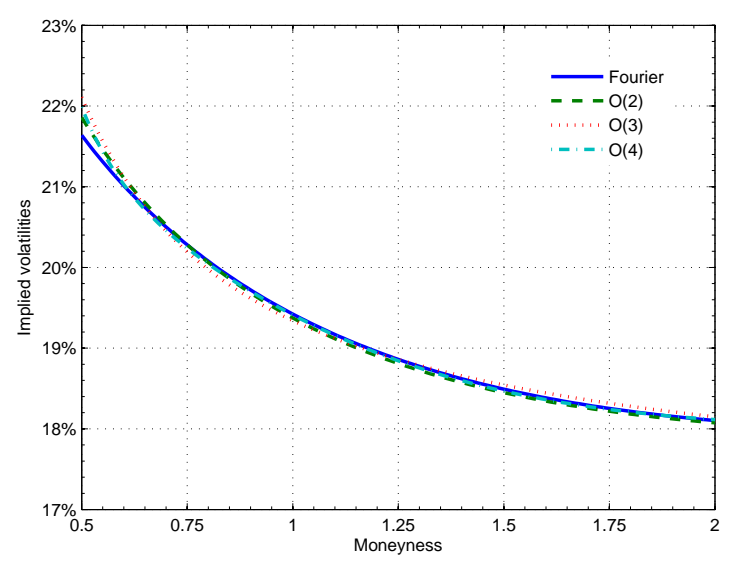

(a) $\tau=5$ years

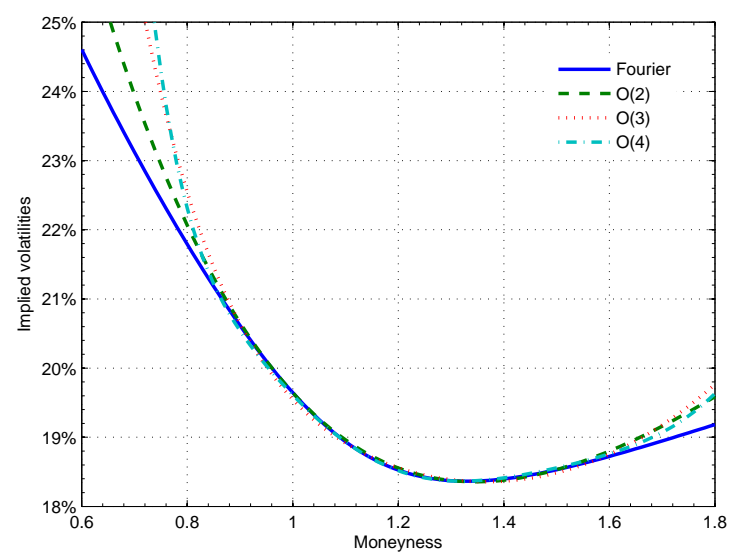

(c) $\tau=1 / 2$ year

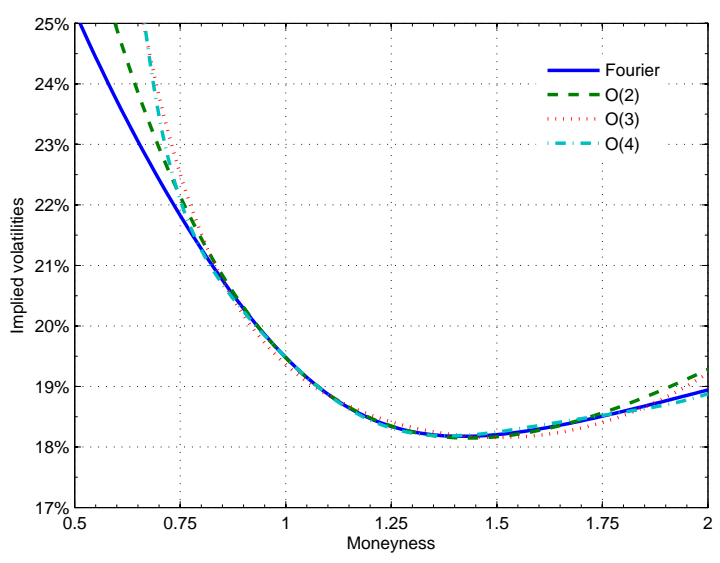

(b) $\tau=1$ year

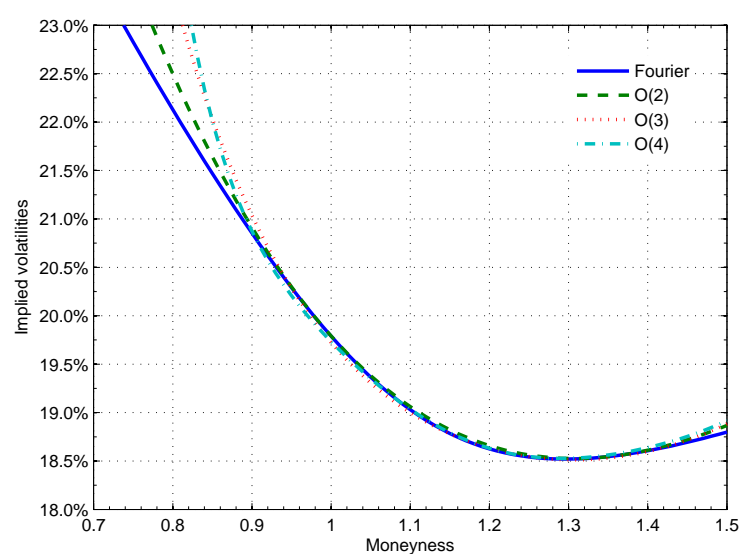

(d) $\tau=1 / 4$ year

Figure 2.1: Implied volatilities as a function of moneyness $K / S_{0}$ for the Heston model.

zero the at-the-money implied variance converges to the spot variance $v_{0}$

$$
\mathfrak{I}_{0}(\tau) \rightarrow v_{0} \quad \text { as } \quad \tau \rightarrow 0
$$

while the short-maturity at-the-money skew is independent of $\kappa$ and $\tau$ and converges to

$$
\mathfrak{I}_{1}(\tau) \rightarrow \frac{\rho \lambda}{2} \quad \text { as } \quad \tau \rightarrow 0 .
$$

As a result, the term-structure of Heston implied volatilities induced by the quadratic approximation is consistent with well-known short-maturity asymptotic results derived by, e.g., Medvedev and Scaillet (2007). However, for extreme maturities far away from 
zero, the behavior of the quadratic approximation is bound to deteriorate. It is immediate to verify that the at-the-money implied variance $\mathfrak{I}_{0}(\tau) \rightarrow \infty$ as $\tau \rightarrow \infty$, except for the no-correlation case. In contrast, the true behavior of the Heston volatility smile flattens to a common asymptotic value independent of moneyness (see Lewis (2000), Chapter 6).

For comparison, Figure 2.2 plots implied volatilities for the quadratic approximation as well as the most-likely-path approximation of Gatheral (2006) which is given by

$$
I^{2}(x, \tau) \approx I_{G}^{2}(x, \tau)=\frac{\hat{w}^{\prime}}{\tau}+\rho \lambda \frac{x}{\kappa^{\prime} \tau}\left(1-\frac{1-e^{-\kappa^{\prime} \tau}}{\kappa^{\prime} \tau}\right),
$$

where

$$
\kappa^{\prime}=\kappa-\frac{\rho \lambda}{2} \quad \text { and } \quad \hat{w}^{\prime}=\eta\left(1-\frac{\kappa}{\kappa^{\prime}}\right)\left(\frac{1-e^{-\kappa^{\prime} \tau}}{\kappa^{\prime}}\right)+\frac{\kappa}{\kappa^{\prime}} \eta \tau
$$

and is valid in the special case $v_{0}=\eta$. Approximation (2.33) is, at least in spirit, similar to the one developed here as it aims at capturing the overall shape of the volatility surface. It matches the true term-structure of the at-the-money implied variance and skew both in the very short-term and for long maturities. In Figure 2.3 at-the-money implied volatilities and implied variance skews are plotted against the quadratic approximation and the mostlikely-path approximation.
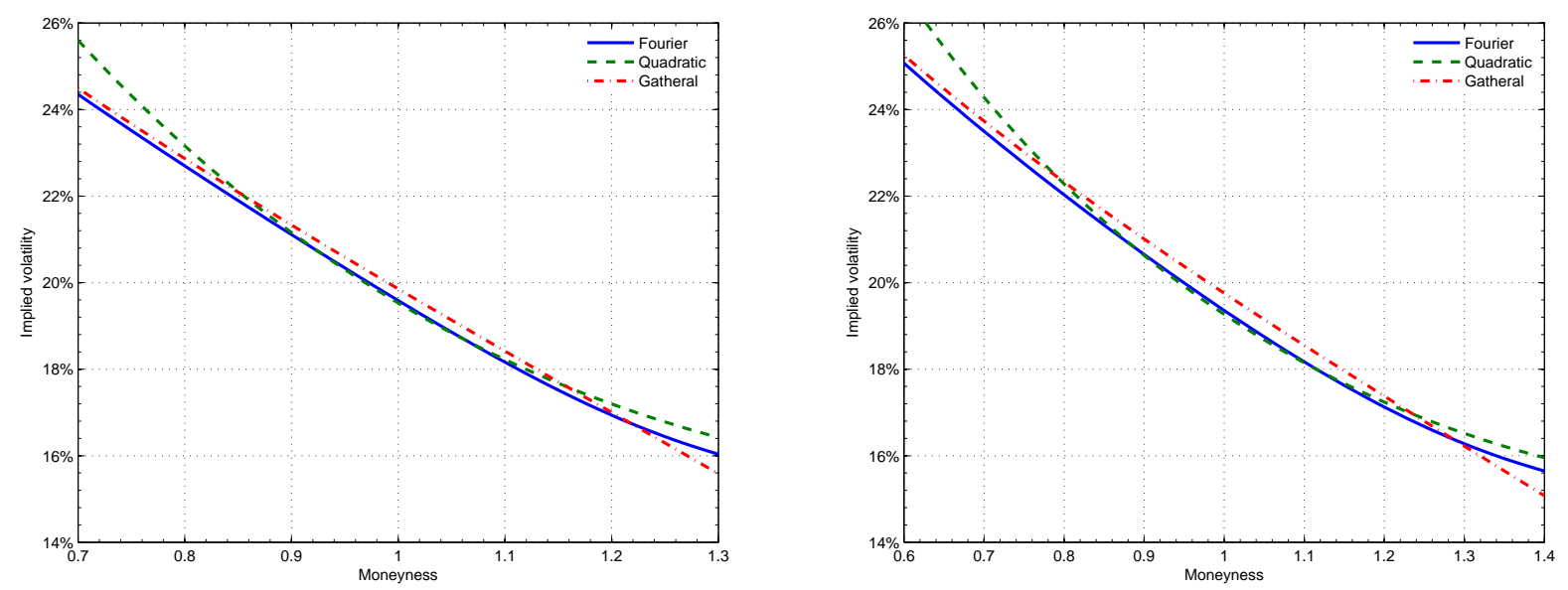

Figure 2.2: Implied volatility as a function of moneyness for the Heston model. 6-month options (Left panel) and 1-year options (right panel).

Figure 2.3 shows that the at-the-money volatilities $\mathfrak{I}_{0}(\tau)$ predicted by the quadratic approximation follow very closely the true term-structure in the short-end and intermediate part. Only for extreme maturities and beyond any option liquidity does it start 

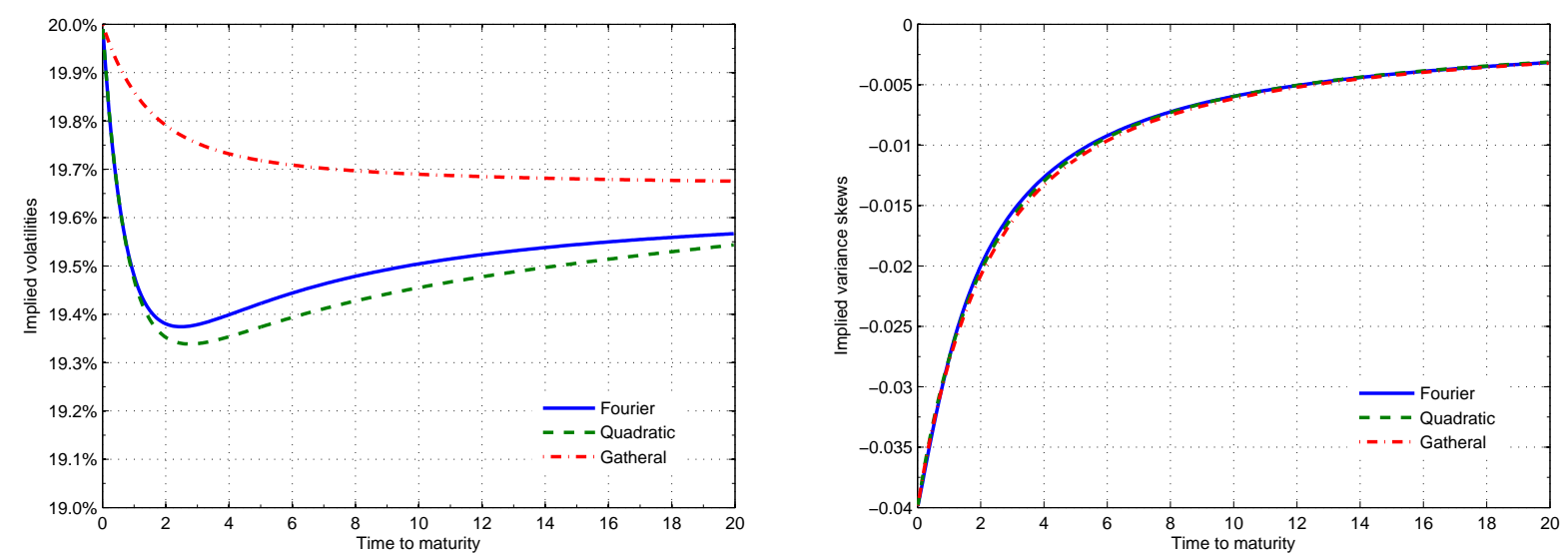

Figure 2.3: At-the-money implied volatility (Left panel) and at-the-money implied variance skew (right panel) as a function of time-to-expiry $\tau$ for the Heston model.

to depart from the true level. While Gatheral's approximation matches the true term structure in the short and long ends, the quadratic approximation is clearly superior in terms of accuracy between these two extremes. Also, the smaller the correlation $\rho$, the more accurate is the approximation and the more extreme are the maturities before it starts to deviate.

Finally, we observe that both approximations capture the term-structure of the at-themoney implied variance skew extremely well. The high accuracy offered by the quadratic approximation, even for long maturities, is anticipated as the variance skew approximation $\mathfrak{I}_{1}(\tau)$ is inversely proportional to the maturity $\tau$ in agreement with the true Heston longdated behavior, see e.g. Forde et al. (2010).

\subsubsection{VG Dynamics}

We now turn to the analysis of expansion (2.17) for the class of exponential Lévy models. In particular, we examine in details the Variance Gamma (VG) model introduced by Madan and Seneta (1990) and further developed in Madan et al. (1998). The VG model is obtained by specifying the effective variance process in (2.9) as a subordinator $Z$ with unit-time Gamma law. The typical parametrization is in terms of the constants $\sigma>0$, $\nu>0$ and $\theta \in \mathbb{R}$ where $\mathbb{E} Z_{1}=\sigma^{2}$ and $\operatorname{Var} Z_{1}=\sigma^{4} \nu$ while $\theta$ is related to the skewness parameter by $c=\theta / \sigma^{2}+1 / 2$. The Lévy exponent of $Z$ is then given by

$$
\kappa(\zeta)=\log \left(1-\zeta \nu \sigma^{2}\right)^{-1 / \nu}
$$


which substituted in (2.10) yields an analytical expression of the effective Laplace transform $\mathcal{L}_{t}^{\text {eff }}$. From this, the moments of the quadratic approximation (2.20) can be derived explicitly

$$
\begin{aligned}
\mathbb{E}\left[V_{\tau}^{e f f}\right] & =\sigma^{2} \tau, & & \operatorname{Var}\left[V_{\tau}^{e f f}\right]=\nu \sigma^{4} \tau, \\
\mathbb{C o v}\left[S_{\tau}^{e f f}, V_{\tau}^{\text {eff }}\right] & =S_{0}\left(\frac{\psi}{1-\psi}\right) \sigma^{2} \tau, & & \operatorname{Var}\left[S_{\tau}^{\text {eff }}\right]=S_{0}^{2}\left(\frac{(1-\psi)^{2}}{1-2 \psi}\right)^{\frac{\tau}{\nu}}-S_{0}^{2},
\end{aligned}
$$

where $\psi=\nu \theta+\frac{1}{2} \nu \sigma^{2}$ which becomes $\psi=c \nu \sigma^{2}$ in terms of the skewness parameter $c$.

Figure 2.4 plots the implied volatilities and their approximations for times to maturity $\tau=5,1,1 / 2,1 / 4$. We use the parameters $\sigma=0.25, \nu=0.10, \theta=-0.25$ from Jäckel (2009). Let us first observe from Figure 2.4 that the approximations are fairly accurate over any reasonable strike range. For instance, for 6 months to maturity, the mean absolute error across the range of $30 \%$ in-the-money to $60 \%$ out-of-the-money options is only 13 volatility basis points. However, Figure 2.4 also makes it clear that the strike range of validity of the approximations shrinks as time-to-maturity decreases. In addition, we observe that the quadratic approximation is the most accurate in the at-the-money region compared to the higher-order approximations.

In terms of the VG model parameters, the term-structure of the at-the-money implied variance is given by

$$
\mathfrak{I}_{0}(\tau)=\sigma^{2}+\frac{\sigma^{2}}{2}\left(\frac{\psi}{1-\psi}\right)-\frac{\nu \sigma^{2}}{16}+\frac{1}{\tau}\left(\left(\frac{(1-\psi)^{2}}{1-2 \psi}\right)^{\frac{\tau}{\nu}}-1\right)-\frac{1}{\tau} \frac{\nu \sigma^{2}}{4},
$$

while the at-the-money variance skew and convexity take on the simple expressions

$$
\mathfrak{I}_{1}(\tau)=\frac{1}{\tau} \frac{\psi}{1-\psi} \quad \text { and } \quad \mathfrak{I}_{2}(\tau)=\frac{1}{\tau^{2}} \frac{\nu}{4} .
$$

This way the approximation links the model-parameters to the key features of the volatility surface. Specifically, $\sigma$ affects the overall level of volatility, $\theta$ governs the skewness through $\psi$, while the parameter $\nu$ is the sole responsible for the convexity.

For comparison, in Figure 2.5, implied volatilities of the quadratic approximation are plotted against the VG approximation of Jäckel (2009) which is based on a singular expansion of the Gamma density for small values of $\nu$. Truncated to the first order, Jäckel's approximation yields the following expression for the implied volatility

$$
I^{2}(x, \tau) \approx I_{J}^{2}(x, \tau) \approx\left(\sigma+\sigma_{1} \nu\right)^{2}
$$




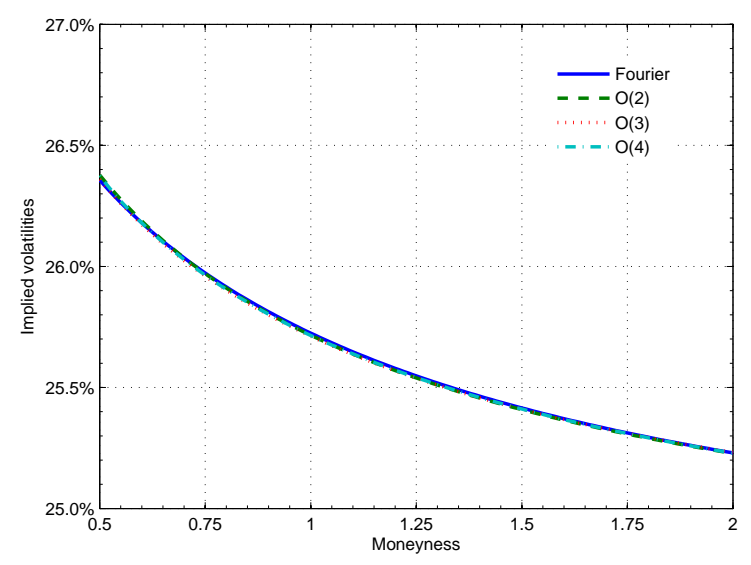

(a) $\tau=5$ years

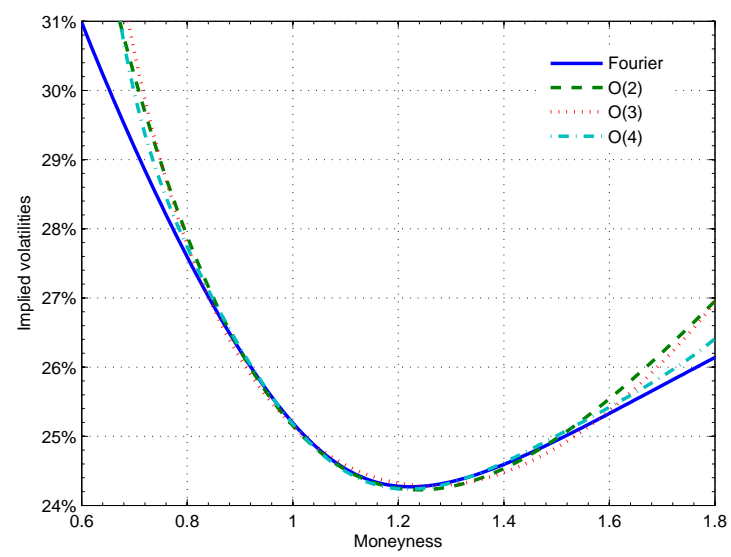

(c) $\tau=1 / 2$ year

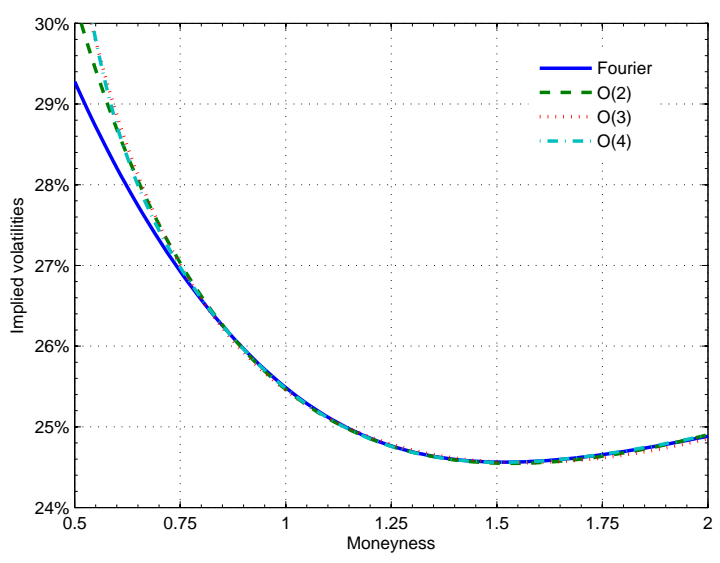

(b) $\tau=1$ year

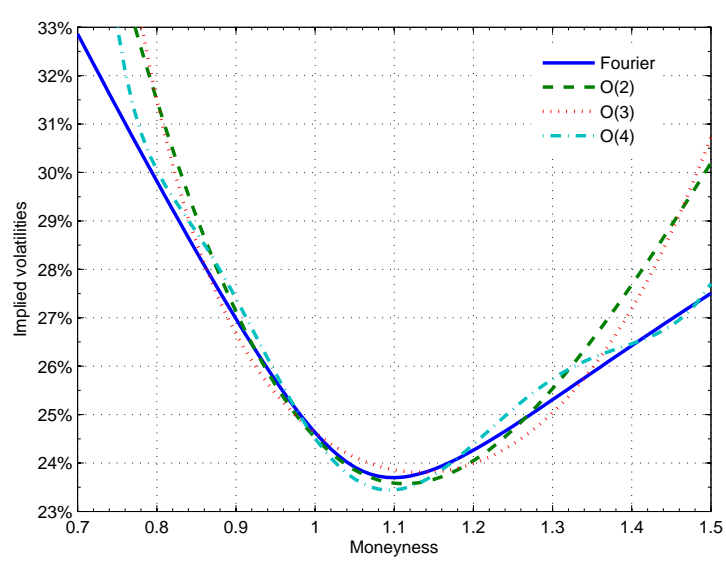

(d) $\tau=1 / 4$ year

Figure 2.4: Implied volatilities as a function of moneyness $K / S_{0}$ for the VG model.

where

$$
\sigma_{1}=\frac{x^{2}}{8 \sigma \tau^{2}}-\frac{\sigma}{8}\left(\frac{\sigma^{2}}{4}+\frac{1}{\tau}\right)+\left(\frac{\sigma}{4}+\frac{x}{2 \sigma \tau}\right) c \nu+\frac{c^{2}}{2 \sigma}
$$

with $x$ denoting as usual the log-moneyness and $c$ being the skewness parameter. Figure 2.6 illustrates the behavior of the at-the-money term structure of implied volatilities and implied variance skews over both short and long time-horizons.

From maturities of around two months up to ten years, the at-the-money volatilities $\mathfrak{I}_{0}(\tau)$ and skews $\mathfrak{I}_{1}(\tau)$ capture the true term-structure very accurately. Even for short maturities the skew approximation $\mathfrak{I}_{1}(\tau)$ gives a fairly accurate fit, while Jäckel's approximation appears to diverge. Nevertheless, the approximation of the at-the-money volatility 

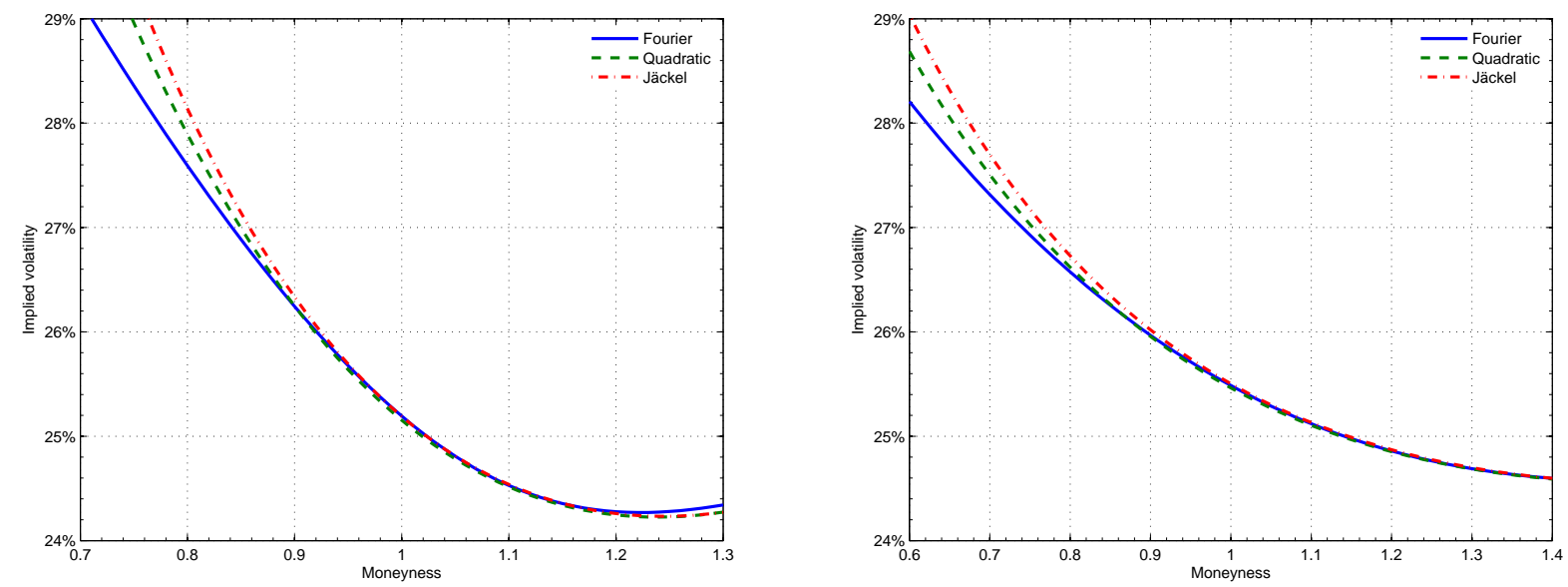

Figure 2.5: Implied volatility as a function of moneyness for the VG model. 6-month options (Left panel) and 1-year options (right panel).

is ill-behaved for short maturities. Both $\mathfrak{I}_{0}(\tau) \rightarrow-\infty$ and $I_{J}^{2}(0, \tau) \rightarrow-\infty$ as $\tau \rightarrow 0$. In contrast, the true behavior of exponential Lévy models force the at-the-money implied volatility to zero at the rate $\sqrt{\tau}$ (Tankov (2011)). However, in spite of this theoretical shortcoming, the approximated curves quite quickly catch up with the true ones.

At the other extreme end of the term-structure a similar issue arises when looking at long-dated smiles. Tehranchi (2009) shows that the implied volatility of exponential Lévy models converges to a constant value independent of moneyness, with the long-term implied variance being approximatively affine in both the log-moneyness $x$ and in the time-to-maturity $\tau$

$$
\tau I^{2}(x, \tau) \approx A \tau+B x+C .
$$

While the at-the-money variance skew and convexity terms (2.37) of the quadratic approximation do imply a flattening at long maturities, the approximated level (2.36) is forced to grow to infinity as $\tau \rightarrow \infty$ whenever the skewness parameter $c \neq 0$. This is due to the contribution of the annualized variance of the effective spot $\operatorname{Var}\left[S_{\tau}^{\text {eff }}\right] / \tau$ which gives

$$
\frac{1}{\tau}\left(\left(\frac{(1-\psi)^{2}}{1-2 \psi}\right)^{\frac{\tau}{\nu}}-1\right) \rightarrow \infty \quad \text { as } \quad \tau \rightarrow \infty .
$$

However, calibration of the VG model to market data typically sets $\psi$ to be a small negative number which yields a very low rate of growth producing only a sizable effect at extremely long maturities. This is also confirmed by Figure 2.6. Thus, in practical 

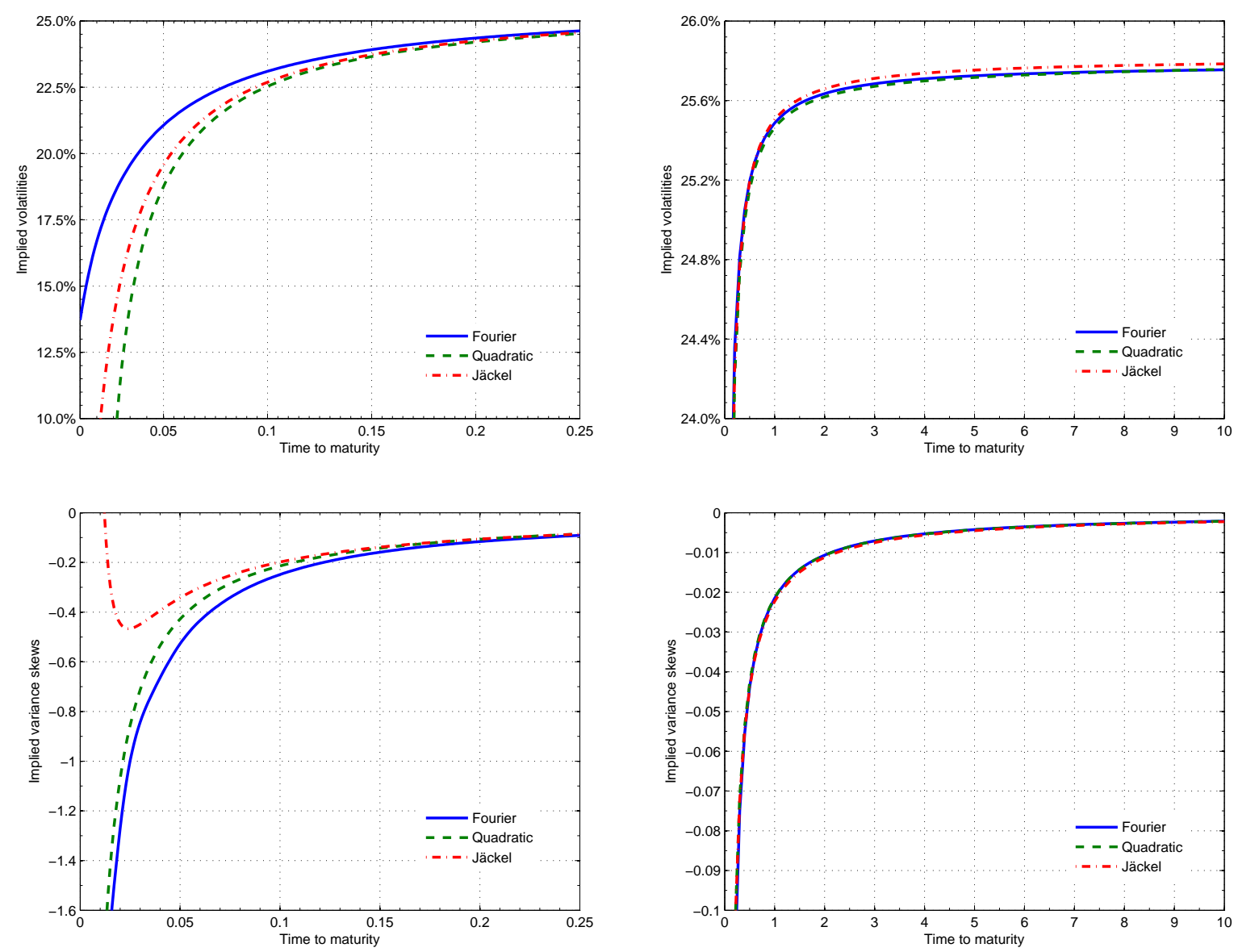

Figure 2.6: At-the-money term structure of implied volatility (top panels) and implied variance skew (bottom panels) for $\tau \in[0 ; 0.25]$ (left panels) and $\tau \in[0.25 ; 10]$ (right panels) in the VG model.

applications the quadratic approximation meets the affine structure (2.39) since the approximation $\mathbb{V} a r\left[S_{\tau}^{e f f}\right] / \tau \approx \operatorname{Var}\left[S_{1}^{e f f}\right]$ is quite accurate for a large interval of long maturities where the effect of the convexity term $\mathfrak{I}_{2}(\tau)$ fades away rather quickly.

\subsection{Applications}

We discuss two potential applications of the proposed expansion of implied volatility. First, the expansion can be used as a control variate in Fourier pricing of options. Second, it may be used as a fast, first-order model calibration approach. In particular, we 
look at joint calibration to the at-the-money volatility and skew. We will exemplify the applications using the VG model and the Heston model. However, any option pricing model embedded in the mixing setup of Section 2.2 admits, in principle, the following applications.

\subsubsection{Control Variate}

Fourier transform methods have been introduced to option pricing by several authors, see e.g. Carr and Madan (1999) or Lewis (2001). Schmelzle (2010) provides a comprehensive overview of Fourier transform methods with applications to option pricing. The approach suggested in Lewis (2001) and independently derived in Lipton (2002) stipulates that given the (normalized) characteristic function $\phi_{\tau}(u) \equiv \mathbb{E} e^{i u \log S_{\tau}}$ the price of a call option is

$$
C(S, K, \tau)=S-\frac{K}{2 \pi} \int_{i \alpha-\infty}^{i \alpha+\infty} e^{-i z x} \phi_{\tau}(-z) \frac{d z}{z^{2}-i z}
$$

for $\alpha=\Im z \in(0,1)$, assuming zero interest rates and dividends for simplicity. In practice, one often chooses the integration contour $\alpha=1 / 2$ and after some rearrangements the formula becomes

$$
C(S, K, \tau)=S-\frac{\sqrt{S K}}{\pi} \int_{0}^{\infty} \Re\left[e^{-i u x} \phi_{\tau}\left(u-\frac{i}{2}\right)\right] \frac{d u}{u^{2}+\frac{1}{4}} .
$$

To improve convergence, Andersen and Andreasen (2002) suggest incorporating a control variate when computing the inverse Fourier integral in (2.42). This idea has been further elaborated in Sepp (2003), Itkin (2005), and Andersen and Piterbarg (2011). The common approach is to use the Black-Scholes price as a control variate. Specifically, using the Fourier transform, one computes the difference between the model price of the option and the Black-Scholes price for the same option characteristics and a suitable volatility. The price of a call option would then be given by

$$
\begin{aligned}
C(S, K, \tau)= & C^{B S}(S, K, \tau ; \sigma) \\
& -\frac{\sqrt{S K}}{\pi} \int_{0}^{\infty} \frac{1}{u^{2}+\frac{1}{4}} \Re\left[e^{-i u x}\left(\phi_{\tau}\left(u-\frac{i}{2}\right)-e^{-\frac{1}{2}\left(u^{2}+\frac{1}{4}\right) \tau \sigma^{2}}\right)\right] d u .
\end{aligned}
$$

Effectively, given a certain accuracy or error tolerance, this reduces the number of function evaluations required in the numerical integration scheme. However, this strategy also poses a tautological question as to which volatility $\sigma$ to use in the Black-Scholes pricing 
formula. Thus, the choice of volatility has generally been more or less arbitrary; a popular choice being the at-the-money spot volatility.

Instead, we suggest using the quadratic approximation to compute an approximate value for the option's implied volatility. In other words, we set $\sigma=I_{Q}(x, \tau)$ in $(2.43)$ with $I_{Q}$ denoting the approximated implied volatility obtained by $(2.20)$. We refer to this approach as the $Q A$ control. As documented in the previous section, the quadratic approximation is quite accurate for such applications. The QA control will further reduce the numerical integration error compared to the common case of arbitrarily chosen volatility, the $B S$ control. The QA approach does not really add more complexity to the problem as the quadratic approximation is just an elementary function of the option's moneyness and expiry. In addition, by appropriate vectorization, the whole implied volatility surface can be approximated in just one sweep providing us with a surface of suitable control variates. Finally, the QA control variate may also be used in model calibration based on Fourier inversion. No extra parameters need to be calibrated as the parameters of the quadratic approximation are exactly the same as those defining the model's characteristic function.

In the numerical experiments, we compare three cases: (i) Fourier pricing without a control variate, (ii) Fourier pricing with the BS control, and (iii) Fourier pricing with the QA control. Considering 1-year call options, Table 2.1 reveals the number of function evaluations required for numerical integration of the inverse Fourier transform across strikes under both VG and Heston dynamics. In the BS control case the Black-Scholes volatility is put equal to the $\sigma$ parameter in the VG case or $\sqrt{v_{0}}$ in the Heston case. We use two adaptive quadratures for the numerical integration, a recursive Simpson's rule and a Gauss-Lobatto quadrature rule, and in both schemes we choose an absolute error tolerance of 1.0e-9. Compared to the case without a control variate, the QA control variate reduces the average number of function evaluations by $64 \%$ (VG) and $63 \%$ (Heston) for Simpson's rule and more than 59\% (VG) and 58\% (Heston) for the Gauss-Lobatto quadrature.

Table 2.2 provides further evidence on the numerical performance of the QA control for 6-month call options. Again, we observe the overall picture is that the QA control reduces the number of function evaluations by $>60 \%$ (Simpson's) and $>50 \%$ (GaussLobatto), on average, compared to not using a control variate at all, while the average reductions are $>20 \%$ (Simpson's) and $>30 \%$ (Gauss-Lobatto) compared to using the BS control. 
Table 2.1: Number of function evaluations required for numerical integration of the inverse Fourier transform for 1-year options

\begin{tabular}{lrrrrrrr}
\hline \hline & \multicolumn{3}{c}{ VG model $^{\mathrm{a}}$} & & \multicolumn{3}{c}{ Heston model $^{\mathrm{b}}$} \\
\cline { 2 - 3 } \cline { 6 - 7 } Strike & No Control & BS Control & QA Control & No Control & BS Control & QA Control \\
\hline 600 & 397 & 225 & 189 & 429 & 253 & 209 \\
700 & {$[618]$} & {$[408]$} & {$[318]$} & {$[618]$} & {$[408]$} & {$[348]$} \\
& 397 & 209 & 153 & & 421 & 217 & 169 \\
800 & {$[618]$} & {$[408]$} & {$[288]$} & {$[618]$} & {$[408]$} & {$[288]$} \\
& 397 & 197 & 141 & & 413 & 201 & 157 \\
900 & {$[588]$} & {$[378]$} & {$[228]$} & {$[588]$} & {$[348]$} & {$[288]$} \\
& 401 & 189 & 109 & & 413 & 197 & 113 \\
1000 & {$[558]$} & {$[318]$} & {$[168]$} & {$[588]$} & {$[318]$} & {$[198]$} \\
& 401 & 189 & 125 & & 417 & 197 & 121 \\
1100 & {$[588]$} & {$[348]$} & {$[198]$} & & {$[588]$} & {$[288]$} & {$[198]$} \\
& 405 & 189 & 141 & & 421 & 201 & 145 \\
1200 & {$[618]$} & {$[348]$} & {$[228]$} & {$[618]$} & {$[318]$} & {$[198]$} \\
& 393 & 185 & 133 & & 405 & 193 & 149 \\
1300 & {$[618]$} & {$[378]$} & {$[258]$} & {$[618]$} & {$[348]$} & {$[258]$} \\
& 405 & 197 & 141 & & 417 & 201 & 145 \\
1400 & {$[618]$} & {$[378]$} & {$[258]$} & {$[618]$} & {$[348]$} & {$[228]$} \\
& 413 & 221 & 161 & & 425 & 213 & 173 \\
& {$[618]$} & {$[378]$} & {$[288]$} & {$[618]$} & {$[378]$} & {$[318]$} \\
\hline Av. change & & $50 \%$ & $64 \%$ & & $50 \%$ & $63 \%$ \\
& & {$[39 \%]$} & {$[59 \%]$} & & {$[42 \%]$} & {$[58 \%]$} \\
\hline \hline
\end{tabular}

Remark: $\tau=1$ year and $S_{0}=1000$. We consider two adaptive quadrature rules: the recursive Simpson's rule (without brackets) and the Gauss-Lobatto quadrature (within brackets). In all cases, we use an absolute error tolerance of 1.0e-9.

${ }^{a}$ VG parameters $\sigma=0.25, \nu=0.10, \theta=-0.25$. The volatility in BS Control is put equal to $\sigma$.

${ }^{\mathrm{b}}$ Heston parameters $\kappa=1.15, \theta=0.04, \eta=0.2, \rho=-0.40, v_{0}=0.04$. The volatility in BS Control is put equal to $\sqrt{v_{0}}$.

${ }^{c}$ Average reduction in function evaluations compared to the No Control case.

\subsubsection{Calibration}

We propose a fast, first-order calibration approach. Specifically, we propose to jointly calibrate the model to the at-the-money volatility and skew using the corresponding approximations presented in this paper. A series of calibration experiments are conducted to document the use of the approach. We consider four trials where we calibrate a given model to data generated from the model itself using known parameters. This allows us 
Table 2.2: Number of function evaluations required for numerical integration of the inverse Fourier transform for 6-month options

\begin{tabular}{|c|c|c|c|c|c|c|}
\hline \multirow[b]{2}{*}{ Strike } & \multicolumn{3}{|c|}{ VG model ${ }^{\mathrm{a}}$} & \multicolumn{3}{|c|}{ Heston model $^{\mathrm{b}}$} \\
\hline & No Control & BS Control & QA Control & No Control & BS Control & QA Control \\
\hline \multirow[t]{2}{*}{600} & 449 & 257 & 249 & 457 & 265 & 233 \\
\hline & {$[648]$} & {$[438]$} & {$[378]$} & {$[648]$} & {$[438]$} & {$[378]$} \\
\hline \multirow[t]{2}{*}{700} & 437 & 237 & 181 & 445 & 233 & 185 \\
\hline & [648] & {$[408]$} & {$[288]$} & {$[648]$} & {$[378]$} & [318] \\
\hline \multirow[t]{2}{*}{800} & 421 & 201 & 161 & 433 & 217 & 165 \\
\hline & {$[618]$} & {$[348]$} & [288] & [648] & {$[378]$} & [258] \\
\hline \multirow[t]{2}{*}{900} & 421 & 201 & 121 & 425 & 181 & 129 \\
\hline & {$[588]$} & {$[318]$} & [198] & [618] & {$[318]$} & [228] \\
\hline \multirow[t]{2}{*}{1000} & 429 & 201 & 121 & 433 & 185 & 117 \\
\hline & {$[588]$} & {$[318]$} & [198] & {$[588]$} & {$[258]$} & [198] \\
\hline \multirow[t]{2}{*}{1100} & 429 & 205 & 145 & 433 & 185 & 125 \\
\hline & {$[618]$} & {$[318]$} & [198] & {$[618]$} & {$[288]$} & [198] \\
\hline \multirow[t]{2}{*}{1200} & 421 & 197 & 161 & 433 & 205 & 153 \\
\hline & {$[618]$} & {$[318]$} & {$[258]$} & {$[648]$} & {$[348]$} & [198] \\
\hline \multirow[t]{2}{*}{1300} & 433 & 205 & 161 & 445 & 213 & 161 \\
\hline & {$[618]$} & {$[318]$} & {$[258]$} & {$[648]$} & {$[318]$} & [288] \\
\hline \multirow[t]{2}{*}{1400} & 441 & 229 & 169 & 453 & 225 & 177 \\
\hline & {$[618]$} & {$[378]$} & {$[258]$} & [648] & {$[378]$} & [318] \\
\hline \multirow[t]{2}{*}{ Av. change ${ }^{c}$} & & $50 \%$ & $62 \%$ & & $52 \%$ & $65 \%$ \\
\hline & & {$[43 \%]$} & {$[58 \%]$} & & $46 \%$ & $58 \%$ \\
\hline
\end{tabular}

Remark: $\tau=1 / 2$ year and $S_{0}=1000$. We consider two adaptive quadrature rules: the recursive Simpson's rule (without brackets) and the Gauss-Lobatto quadrature (within brackets). In all cases, we use an absolute error tolerance of 1.0e-9.

${ }^{a}$ VG parameters $\sigma=0.25, \nu=0.10, \theta=-0.25$. The volatility in BS Control is put equal to $\sigma$.

${ }^{\mathrm{b}}$ Heston parameters $\kappa=1.15, \theta=0.04, \eta=0.2, \rho=-0.40, v_{0}=0.04$. The volatility in BS Control is put equal to $\sqrt{v_{0}}$.

${ }^{c}$ Average reduction in function evaluations compared to the No Control case.

to evaluate how well the calibration procedure captures the true parameters of the data. In the first two trials, we calibrate the VG model to data on at-the-money volatilities and skews generated using known parameters from (i) Jäckel (2009) and (ii) Madan et al. (1998). In the last two trials we turn to the Heston model and consider calibration to data generated based on parameters from (i) Forde et al. (2012) and (ii) Bakshi et al. (1997). All data for the calibrations were generated using the Fourier transform approach dis- 
cussed in the previous section. First, note that we have the following implicit relationship between the implied volatility surface and the characteristic function,

$$
\int_{0}^{\infty} \Re\left[e^{-i u x}\left(\phi_{\tau}\left(u-\frac{i}{2}\right)-e^{-\frac{1}{2}\left(u^{2}+\frac{1}{4}\right) \tau I^{2}}\right)\right] \frac{d u}{u^{2}+\frac{1}{4}}=0,
$$

allowing us to numerically compute the true implied volatilities. Then, following Gatheral (2006), we compute the at-the-money implied volatility skew as

$$
\left.\frac{\partial I}{\partial x}\right|_{x=0}=-e^{\frac{\tau I^{2}}{8}}\left(\frac{2}{\pi \tau}\right)^{\frac{1}{2}} \int_{0}^{\infty} \frac{u}{u^{2}+\frac{1}{4}} \Im\left[\phi_{\tau}\left(u-\frac{i}{2}\right)\right] d u .
$$

Next, we use our quadratic approximation to calibrate the models to the generated atthe-money volatilities and at-the-money skew values jointly. We apply the LevenbergMarquardt algorithm to minimize the sum of squared errors. In the calibration we have option maturities of one month up to five years, i.e., $\tau \in(1 \mathrm{M}, 2 \mathrm{M}, 3 \mathrm{M}, 6 \mathrm{M}, 9 \mathrm{M}, 12 \mathrm{M}$, $18 \mathrm{M}, 24 \mathrm{M}, 36 \mathrm{M}, 48 \mathrm{M}, 60 \mathrm{M})$. This leaves us with $2 \times 11$ observation points to calibrate to.

\begin{tabular}{|c|c|c|c|c|}
\hline \multicolumn{5}{|l|}{ VG model } \\
\hline$\sigma$ & 0.2500 & $0.2523^{\mathrm{a}}$ & 0.1213 & $0.1226^{\mathrm{a}}$ \\
\hline$\nu$ & 0.1000 & $0.1164^{\mathrm{a}}$ & 0.1686 & $0.1725^{\mathrm{a}}$ \\
\hline$\theta$ & -0.2500 & $-0.2399^{\mathrm{a}}$ & -0.1436 & $-0.1393^{\mathrm{a}}$ \\
\hline \multicolumn{5}{|c|}{ Heston model } \\
\hline & \multicolumn{2}{|c|}{ Forde et al. (2012) } & \multicolumn{2}{|c|}{ Bakshi et al. (1997) } \\
\hline$\kappa$ & 1.1500 & $1.2155^{\mathrm{a}}$ & 1.1500 & $1.5321^{\mathrm{a}}$ \\
\hline$\eta$ & 0.0400 & $0.0402^{\mathrm{a}}$ & 0.0348 & $0.0337^{\mathrm{a}}$ \\
\hline$\lambda$ & 0.2000 & $0.2142^{\mathrm{a}}$ & 0.3900 & $-0.4142^{\mathrm{a}}$ \\
\hline$\rho$ & -0.4000 & $-0.3796^{\mathrm{a}}$ & -0.6400 & $-0.6313^{a}$ \\
\hline$v_{0}$ & 0.0400 & $0.0401^{\mathrm{a}}$ & 0.0348 & $0.0347^{\mathrm{a}}$ \\
\hline
\end{tabular}

Table 2.3: Model calibration results

a Parameters obtained by model calibration.

Table 2.3 provides the calibrated as well as the true parameters in the four cases. We see that the suggested expansion-based calibration approach gives parameter values that are reasonably close to the true parameters from which the data was generated. In the case of the VG model the $\theta$ seems to be the most troublesome parameter to capture, while 
for the Heston model $\kappa$ appears to be the trickiest. Besides a gain in computational speed, the advantage of the calibration approach is that fewer data points are required in the calibration compared to calibrating to an entire option price surface. Consistency with the observed at-the-money volatilities controls the level of the model implied volatility surface, while consistency with the at-the-money skews secures that the model volatility surface has the correct smile/skew shape.

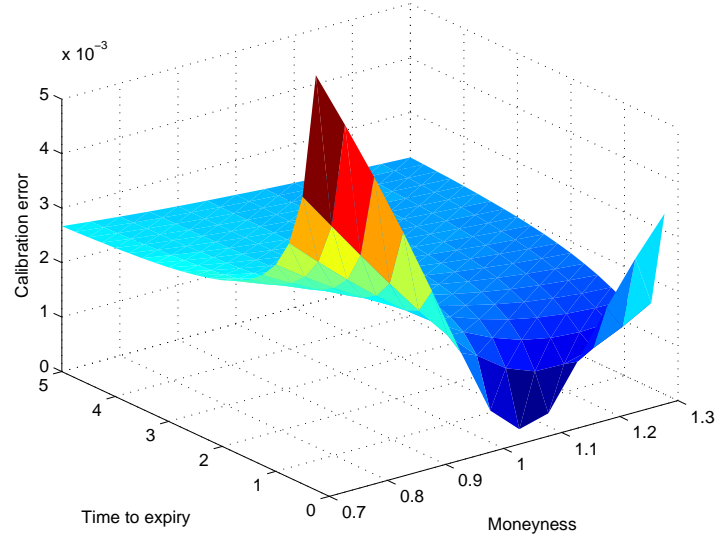

(a)

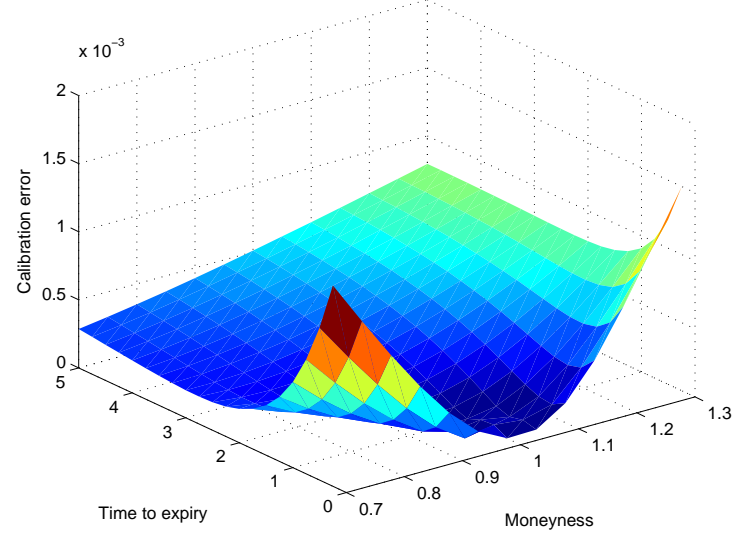

(c)

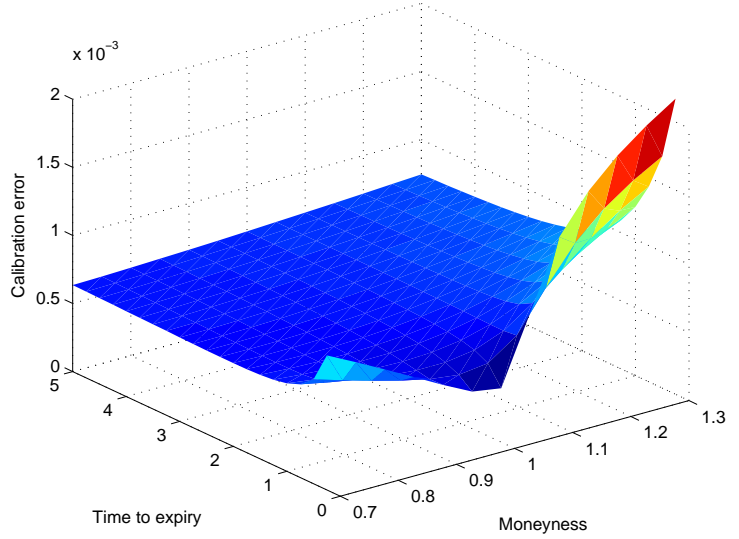

(b)

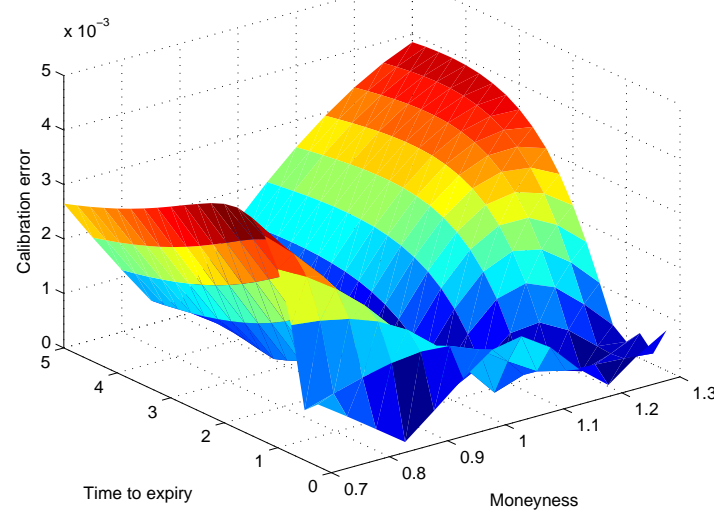

(d)

Figure 2.7: Surfaces of absolute calibration errors in implied volatility. (a) VG model with Jäckel (2009) parameters. (b) VG model with Madan et al. (1998) parameters. (c) Heston model with Forde et al. (2012) parameters. (d) Heston model with Bakshi et al. (1997) parameters.

To further support the calibrated parameters, we look at the absolute errors between 
the true implied volatilities and implied volatilities based on the calibrated parameters for call options with strikes from $30 \%$ out-of-the-money to $30 \%$ in-the-money. The calibration errors are illustrated in Figure 2.7. In all four cases the mean absolute calibration errors are less than 10 volatility basis points. Even for liquid equity options, the bid-ask spread is often not much less than 100 volatility basis points. In other words, the differences between the true and the calibrated parameters appear economically insignificant.

\subsection{Conclusion}

In this paper we derive a closed-form expansion of the implied volatility surface. The expansion is simple and easy to interpret and implement. In addition, it decomposes the volatility surface into meaningful quantities that directly relate its smile/skew shape to higher-order option risks and analytical features of the underlying model. We apply the expansion to, for example, the Heston stochastic volatility model and the pure-jump Variance Gamma model. However, the expansion is largely generic in the sense that it may be used for a broad class of option pricing models displaying both jumps and stochastic volatility in the underlying dynamics. Finally, we explore two domains of potential application. First, we suggest using the expansion as a control variate in Fourier option pricing. Our numerical experiments show that this effectively reduces the number of function evaluations required in the numerical integration procedure. Secondly, we propose a speedy, expansion-based approach for model calibration to at-the-money implied volatilities and skews. 


\section{Chapter 3}

The Impact of Jump Distributions on the

Volatility of Variance 


\title{
The Impact of Jump Distributions on the Volatility of Variance
}

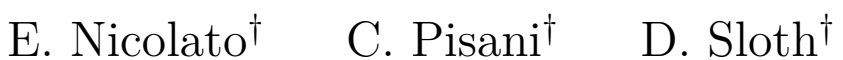

$\dagger$ Department of Economics and Business, Aarhus University, Aarhus

\begin{abstract}
We consider a tractable affine stochastic volatility model that generalizes the seminal Heston (1993) by augmenting it with jumps in the instantaneous variance. In this framework, we consider options written on the realized variance and we examine the impact of the distribution of jumps on the associated implied volatility smile. The model allows us to isolate the unique impact of the jump distribution. We provide sufficient conditions for the asymptotic behavior of the volatility of variance for small and large strikes. In particular, we show that by selecting alternative jump distributions, one obtains fundamentally different shapes of the implied volatility of variance smile - some clearly at odds with the upward-sloping volatility skew observed in variance markets.
\end{abstract}

\subsection{Introduction}

The empirical literature offers ample evidence on the presence of jumps in volatility. From a derivatives perspective, Duffie et al. (2000) were among the first to address the issue of random volatility jumps. The authors proposed a double-jump model within the affine diffusion class which presumes jumps in both asset prices and their variances. Since then, this model has been applied empirically in a number of studies including Broadie et al. (2007), Chernov et al. (2003), Eraker et al. (2003), and Eraker (2004) among others. These studies generally find evidence for jumps both in the price level and its volatility. Also, several authors have considered the pricing of volatility derivatives in 
models with variance jumps. Among them Sepp (2008a), Sepp (2008b), and Lian and Zhu (2011) proposed augmenting the popular square-root dynamics of Heston (1993) to allow for exponential jumps in the instantaneous variance. Nevertheless, despite the common notion to include jumps in the variance dynamics, an examination of the distribution of jumps and its financial implications appear - to the best of our knowledge - curiously absent from the literature. Here we show that the particular distribution of jumps does, in fact, have a profound impact on the behavior of the implied volatility smile of variance.

In contrast to the downward-sloping skew of implied volatilities in equity markets, the volatility of variance has an upward-sloping skew. The financial explanation of this comes from the empirical fact that large losses in the price of the underlying are usually followed by high levels of volatility (Sepp (2008b)). This means that out-of-the-money call options on variance provide protection against market crashes. To compensate for the insurance risk, the call option writer will charge a premium accordingly, very much like the writer of put options on the stock or index itself. This market behavior cannot be captured by any kind of distribution of the jumps in the instantaneous variance dynamics. In fact, some jump distributions imply a shape of the volatility of variance that is clearly at odds with the upward-sloping volatility smile. Evidently, correctly grasping the true distribution of variance jumps is of paramount importance for trading and risk management of variance and volatility derivatives. This paper attempts to fill this gap.

We consider a tractable affine stochastic volatility model that generalizes the seminal model of Heston (1993) by allowing for jumps in the instantaneous variance. This model can also be seen as a particular instance of the double-jump model of Duffie et al. (2000). In this framework, we consider options written on the realized variance and we examine the impact of the distribution of jumps on the associated implied volatility smile. Existing studies on pricing of realized variance options include Carr et al. (2005), Drimus (2012), and Sepp (2008a), while Cont and Kokholm (2011) develop a flexible market model for consistent valuation of realized variance derivatives, index options, and VIX derivatives.

Our model allows for an enormous variety of distributions for the jumps in the instantaneous variance dynamics, predicting completely different shapes and characteristics of the implied volatility of variance surface. Furthermore, a particularly neat subclass of the model includes the case when the instantaneous variance is driven by a pure-jump Ornstein-Uhlenbeck (OU) type process (Barndorff-Nielsen and Shephard (2001)). In this case, the realized variance is driven solely by jumps, which allows us to isolate the unique impact of the jump distribution. 
Based on the classical Tauberian theorems and more recent results of Lee (2004) and Gulisashvili (2012) on implied volatility asymptotics, we provide a direct link between the tail behavior of the jump distribution, the distribution of realized variance, and the impact on the implied volatility smile of variance. We derive easy-to-check sufficient conditions for the asymptotic behavior of volatility of variance for small as well as large strikes, given the particular distribution of variance jumps. In particular, we show that for a number of specific jump distributions one obtains fundamentally different shapes of the implied volatility of variance. For example, we find that the Gamma distribution leads to a downward-sloping volatility skew, the Inverse Gamma distribution predicts an upward-sloping skew, while the Inverse Gaussian distribution leads to a frown of the implied volatility surface.

The rest of the paper is organized as follows: In Section 3.2 we give the necessary background on realized variance options and a description of our affine stochastic volatility model with jumps in the instantaneous variance. In Section 3.3 we provide some preliminary results on wing asymptotics for a general distribution of the realized variance. In Section 3.4 we consider pure-jump OU model specifications and derive sufficient conditions for the asymptotic behavior of the volatility of variance. Section 3.5 describes a number of alternative jump distributions and we provide numerical results for selected cases. In Section 3.6 we provide analogous results on the wing asymptotics for full specifications of our affine stochastic volatility model. Finally, Section 3.7 concludes the paper.

\subsection{Options on Realized Variance}

The realized variance of a given asset is a measure of the variation in the price realized over some period of time. More precisely, assume we observe the asset price $S_{t}$ on some regular grid $0=t_{0}<t_{1}<\cdots<t_{n}=T$ fixed to the time interval $[0, T]$, then the realized variance is defined as

$$
R V_{N}=\sum_{n=0}^{N} \log \left(S_{t_{n}} / S_{t_{n-1}}\right)^{2}=\sum_{n=0}^{N}\left(X_{t_{n}}-X_{t_{n-1}}\right)^{2},
$$

where $X_{t}=\log \left(S_{t}\right)$ denotes the log-price. Common practice is to approximate realized variance by the quadratic variation $[X]_{T}$ of the log-price

$$
[X]_{T}=\lim _{\Pi \rightarrow 0} \sum_{n=0}^{N}\left(X_{t_{n}}-X_{t_{n-1}}\right)^{2}
$$


This is justified by the fact that realized variance converges to $[X]_{T}$ as the mesh-size of the time-grid, $\Pi=\sup _{n=1, \ldots, N}\left(t_{n}-t_{n-1}\right)$, goes to zero. As a result, when we talk about options on realized variance we really mean the continuous-time limit of the realized variance which is the quadratic variation of the log-price. In practice, Broadie and Jain (2008) show that the approximation works well for daily data.

We adopt the simplifying assumption of zero interest rates and dividend yields as they have no impact on the realized variance distribution. Furthermore, we assume that under the pricing measure $\mathbb{Q}$ the $\log$-price $X$ and its instantaneous variance $v$ evolve according to the following risk-neutral dynamics

$$
\begin{aligned}
& d X_{t}=-\frac{1}{2} v_{t} d t+\sqrt{v_{t}} d W_{t} \\
& d v_{t}=\lambda\left(\eta-v_{t}\right) d t+\varepsilon \sqrt{v_{t}} d B+d J_{t} .
\end{aligned}
$$

The processes $W$ and $B$ are standard Brownian motions with correlation parameter $\rho$. The jump process $J$ is an increasing and driftless Lévy process independent of $(W, B)$ with finite first moment and Lévy measure $\nu_{J}(d x)$. That is, the cumulant exponent $\kappa_{J}$ is given by

$$
\kappa_{J}(u)=\log \mathbb{E}\left[e^{-u J_{1}}\right]=\int_{0}^{\infty}\left(e^{-u x}-1\right) \nu_{J}(d x)
$$

where $\nu_{J}(d x)$ is a measure on the positive real line satisfying $\int_{0}^{\infty} x \nu_{J}(d x)<\infty$. Finally, the parameters $\lambda, \eta$ and $\varepsilon$ are non-negative constants.

The stochastic volatility model (3.1) generalizes the seminal Heston (1993) model by augmenting the square root process describing the instantaneous variance to allow for jumps. It can also be seen as a particular instance of the double-jump stochastic volatility (SVJJ) model introduced by Duffie et al. (2000) where both the instantaneous variance $v$ and the log-price process $X$ are affected by jumps. To emphasize the fact that in (3.1) jumps are allowed only at the variance level, we refer to the model as SVJ-v.

The main advantage of the SVJ-v model specification is that the quadratic variation coincides with the integrated variance of $X$. Thus, the annualized realized variance denoted by $V$ hereafter - is given by

$$
V_{T}=\frac{1}{T} \int_{0}^{T} v_{t} d t
$$

This means that, on one hand, the SVJ-v model allows us to deal with the integrated variance, a quantity which in affine models is particularly simple and mathematically tractable. On the other hand, it also allows us to explore alternative distributions for the jump process $J$ and to analyze their impact on the price of realized variance derivatives. 
In this work, we focus on call options on the annualized realized variance. For a given maturity $T$, the price $C(K)$ of a call striking at $K$ is given by

$$
C(K)=\mathbb{E}\left(V_{T}-K\right)^{+}
$$

while its implied volatility is obtained by matching the call price with the Black-Scholes formula. More precisely, the implied volatility of variance $I(K)$ is defined as the solution of the following equation

$$
C(K)=V_{0} \Phi\left(\frac{x}{I(K) \sqrt{T}}+\frac{I(K) \sqrt{T}}{2}\right)+K \Phi\left(\frac{x}{I(K) \sqrt{T}}-\frac{I(K) \sqrt{T}}{2}\right)
$$

where $V_{0}=\mathbb{E}\left[V_{T}\right]$ is the fair annualized variance seen at the present time, $x=\log \left(V_{0} / K\right)$ is the $\log$-moneyness of variance and $\Phi(\cdot)$ denotes the cumulative distribution function of a standard normal law.

\subsection{Preliminary Results on Wing Asymptotics}

While the main purpose of this study is to show how the distribution of variance jumps can lead to different behavior of the implied volatility of variance across strikes, we first provide a number of preliminary results on the wing asymptotics of the variance smile for a general distribution of the realized variance $V_{T}$.

The asymptotic behavior of the wings of the implied volatility smile has attracted considerable attention during the last decade. In a ground-breaking paper, Lee (2004) relates the wings of the smile to the number of moments of the underlying distribution and a large part of the literature since then has been devoted to provide refinements and extensions of Lee's moment formulas. Among others, we refer to the studies of Benaim and Friz (2009), Benaim and Friz (2008), Gulisashvili (2009), Gulisashvili (2012) and the references therein.

However, the focus has been on implied volatility smiles for equity options. So, most of the results are formulated in terms of distribution functions or moment generating functions related to the distribution of $\log V_{T}$ as the natural building block in that context is the log-price. In contrast, for variance options the relevant and tractable element is the variance $V_{T}$ itself, which implies that a lot of the previous results available for equity options cannot be directly transferred to the case of variance smiles. 
Still, Lee's moment formulas as well as the work of Gulisashvili (2012) play a crucial role in the subsequent analysis. We summarize the results relevant for our applications in Theorem 3 below. The function $\psi$ appearing in the formulation is given by

$$
\psi(x)=2-4\left(\sqrt{x^{2}+x}-x\right)
$$

and $g(x) \sim h(x)$ means that $g(x) / h(x) \rightarrow 1$ as either $x \rightarrow 0$ or $x \rightarrow \infty$ depending on the context. To keep matters simple, we assume that $I(K)$ is well defined for all $K>0$ and we implicitly postulate the existence of all the involved limits. We refer to Benaim et al. (2008) for a discussion on this issue for equity smiles.

Theorem 3. The following statements hold for the implied volatility of variance $I(K)$

1. Let $\tilde{p}_{V}=\sup \left\{p: \mathbb{E}\left[V_{T}^{p+1}\right]<\infty\right\}$, then

$$
I^{2}(K) \sim \psi(\tilde{p}) \log \left(K / V_{0}\right) / T \quad \text { as } \quad K \rightarrow \infty .
$$

2. Let $\tilde{q}_{V}=\sup \left\{q: \mathbb{E}\left[V_{T}^{-q}\right]<\infty\right\}$, then

$$
I^{2}(K) \sim \psi(\tilde{q}) \log \left(V_{0} / K\right) / T \quad \text { as } \quad K \rightarrow 0 .
$$

3. In the case of $\tilde{p}_{V}=\infty$, then

$$
I(K) \sim \frac{1}{\sqrt{2 T}} \log (K)\left(\log \frac{1}{C(K)}\right)^{-1 / 2} \text { as } K \rightarrow \infty .
$$

4. In the case of $\tilde{q}_{V}=\infty$, then

$$
I(K) \sim \frac{1}{\sqrt{2 T}} \log \frac{1}{K}\left(\log \frac{K}{P(K)}\right)^{-1 / 2} \quad \text { as } K \rightarrow 0,
$$

where $P(K)$ denotes the pricing function of a put option on $V_{T}$ with strike $K$ and maturity $T$.

Formulas (3.3) and (3.4) are derived in Theorem 3.2 and Theorem 3.4 in Lee (2004). While being undoubtedly powerful, they are not informative when $\tilde{p}=\infty$ or $\tilde{q}=\infty$. In those cases, formulas (3.5) and (3.6), which we refer to as Gulisashvili's criterions, turn out to be very useful. Criterion (3.5) for large strikes corresponds to formula (2.8) in 
Gulisashvili (2012) while the corresponding criterion (3.6) for small strikes can be derived following analogous techniques.

As in the case of equity smiles, Karamata's theory of regular variation proves to be a powerful tool in connection with Theorem 3. Recall that a positive, measurable function $f$ is regularly varying at $\infty$ with index $\alpha \in[0, \infty)$ if the following holds

$$
\lim _{x \rightarrow \infty} \frac{f(t x)}{f(x)}=t^{\alpha}, \quad t>0 .
$$

In this case we write $f \in R_{\alpha}$. When $f \in R_{0}$, then we say that $f$ is slowly varying at $\infty$. It can be shown that $f \in R_{\alpha}$ if and only if it takes the following form

$$
f(x)=x^{\alpha} \ell(x)
$$

where $\ell \in R_{0}$.

Karamata's Tauberian theorem relates the regular variation of a function to that of its Laplace transform. More precisely, it can be formulated as follows. Consider rightcontinuous, non-decreasing function $f$ on $\mathbb{R}_{+}$and let

$$
\mathcal{L}_{f}(x)=\int_{0}^{\infty} e^{-x u} d f(u), \quad x>0
$$

denote its Laplace transform. Then, for $\alpha \geq 0$ it holds that

$$
f(x) \in R_{\alpha} \quad \text { if and only if } \quad \mathcal{L}_{f}(1 / x) \in R_{\alpha},
$$

and in this case $\mathcal{L}_{f}(1 / x) \sim \Gamma(1+\alpha) f(x)$ as $x \rightarrow \infty$, where $\Gamma$ is the Gamma function. It can be found, e.g., in Embrechts et al. (1997), Theorem A3.9 on p. 569. For our purposes, it turns out to be more useful to deal with a dual formulation of the above result which instead relates the regular variation of $f(1 / x)$ to that of $\mathcal{L}_{f}(x)$.

Theorem 4 (Karamata's Tauberian Theorem). For a right-continuous, non-decreasing function $f$ on $\mathbb{R}_{+}$and for $\alpha \geq 0$ it holds that

$$
f(1 / x) \in R_{-\alpha} \quad \text { if and only if } \quad \mathcal{L}_{f}(x) \in R_{-\alpha},
$$

and in this case $\mathcal{L}_{f}(x) \sim \Gamma(1+\alpha) f(1 / x)$ as $x \rightarrow \infty$.

The proof of this alternative formulation follows along the same lines as that of the classic Karamata's Tauberian theorem and it is reported in the Appendix for completeness. In what follows, we will also make use of rapidly varying functions. A positive, 
measurable function $f$ is said to be rapidly varying with index $-\infty\left(f \in R_{-\infty}\right)$ if

$$
\lim _{x \rightarrow \infty} \frac{f(t x)}{f(x)}= \begin{cases}0 & \text { if } t>1 \\ \infty & \text { if } 0<t<1\end{cases}
$$

Specifically, we will refer to the fact that if $f \in R_{-\infty}$ and $\alpha \in \mathbb{R}$ then

$$
\lim _{x \rightarrow \infty} \frac{x^{\alpha+1} f(x)}{\int_{x}^{\infty} t^{\alpha} f(t) d t}=\infty
$$

which can be found in, e.g., Embrechts et al. (1997), p. 570.

Let us now turn to our analysis of the asymptotic behavior of volatility of variance at large strikes and at small strikes. We provide sufficient conditions for the right and left wings of the variance smile to be upward or downward-sloping. Here, we denote by $F_{V}$ and $\bar{F}_{V}$ the distribution function and the distribution tail of $V_{T}$,

$$
F_{V}(x)=\mathbb{Q}\left(V_{T} \leq x\right) \quad \text { and } \quad \bar{F}_{V}(x)=1-F_{V}(x),
$$

while we write $\mathcal{L}_{V}$ for its Laplace transform

$$
\mathcal{L}_{V}(u)=\mathbb{E}\left[e^{-u V_{T}}\right]
$$

A straightforward application of the regular variation theory yields the following sufficient condition for the right wing of the variance smile to be upward-sloping.

Proposition 7 (Upward-sloping right wing). Suppose that $\bar{F}_{V} \in R_{-\alpha-1}$ for $\alpha>0$. Then Lee's formula (3.3) for large strikes holds with $\tilde{p}_{V}=\alpha$.

Proof. This follows immediately from the definition of regular variation.

Next, we provide a sufficient condition for the right wing to be downward-sloping.

Proposition 8 (Downward-sloping right wing). Suppose that the distribution tail $\bar{F}_{V}$ is dominated by a Weibull-type tail function, i.e. there exist positive constants $\alpha, \beta, \gamma>0$ such that

$$
\bar{F}_{V}(x) \leq \gamma e^{-\alpha x^{\beta}} \quad \text { for } \quad x \in\left(x_{0}, \infty\right) \text { with } x_{0}>0 .
$$

Then $\tilde{p}_{V}=\infty$ and $I(K) \rightarrow 0$ as $K \rightarrow \infty$. 
Proof. It is sufficient to consider the case $0<\beta \leq 1$. For $K$ large enough, it holds that

$$
C(K)=\int_{K}^{\infty} \bar{F}_{V}(x) d x \leq A \int_{\alpha K^{\beta}}^{\infty} e^{-x} x^{1 / \beta-1} d x=A \Gamma_{1 / \beta}\left(\alpha K^{\beta}\right)
$$

where $A=\gamma \frac{\beta}{\alpha^{1 / \beta}}$ is a positive constant and $\Gamma_{s}(x)$ denotes the lower incomplete Gamma function. Recall that $\Gamma_{s}(x) \sim x^{s-1} e^{-x}$ as $x \rightarrow \infty$ (see e.g. Abramowitz and Stegun (1987), p. 263). The result now follows from Gulisashivili's criterion (3.5) for large strikes, which can be applied as condition (3.10) entails that $\tilde{p}=\infty$.

If the tail condition (3.10) is satisfied with $\beta=1$, then one says that the distribution of $V_{T}$ has a thin tail. This is in fact equivalent to zero being in the interior domain of the Laplace transform $\mathcal{L}_{V}$, i.e.

$$
\bar{F}_{V}(x) \leq \gamma e^{-\alpha x} \quad \text { for } x \geq x_{0} \quad \text { iff } \quad 0 \in \stackrel{\circ}{\mathcal{D}}_{V}
$$

where $\mathcal{D}_{V}=\left\{x \in \mathbb{R}: \mathcal{L}_{V}<\infty\right\}$.

Let us now consider the left wing of the variance smile. In this case, it is possible to obtain a formulation of Lee's formula (3.4) for small strikes in terms of the Laplace transform $\mathcal{L}_{V}$. This is feasible thanks to Karamata's Tauberian Theorem as given in Theorem (4) if we consider distributions enjoying some regular variation property.

Proposition 9 (Upward-sloping left wing). Assume that $F_{V}$ is a continuous function. Suppose that for an $\alpha \geq 0, F_{V}(1 / x) \in R_{-\alpha}$ or equivalently $\mathcal{L}_{V}(x) \in R_{-\alpha}$. Then Lee's formula (3.4) for small strikes holds with index $\tilde{q}_{V}=\alpha$.

Proof. The statement follows immediately by observing that the tail distribution function of $V^{-1}$ is given by

$$
\bar{F}_{V^{-1}}(x)=\mathbb{Q}\left(V^{-1}>x\right)=F_{V}(1 / x)
$$

and therefore $\bar{F}_{V^{-1}} \in R_{-\alpha}$.

We conclude our collection of preliminary results with a sufficient condition for the left wing to be downward-sloping to zero. In this final case, de Bruijn's Tauberian theorem is the key result which allows for a convenient formulation in terms of the Laplace transform $\mathcal{L}_{V}$. Here we report a simplified version as stated in Voss (2009) which is sufficient for our purposes. 
Theorem 5 (de Bruijn's Tauberian Theorem). Let $\alpha \in(0,1)$ and $\beta>0$ be constants satisfying $\frac{1}{\alpha}-\frac{1}{\beta}=1$. Then the limit

$$
\lim _{x \rightarrow 0} x^{\beta} \log F_{V}(x)=s \leq 0
$$

exists if and only if the limit

$$
\lim _{x \rightarrow \infty} \frac{\log \mathcal{L}_{V}(x)}{x^{\alpha}}=r \leq 0
$$

exists. In this case $|\alpha r|^{1 / \alpha}=|\beta s|^{1 / \beta}$.

The left-wing result goes as follows.

Proposition 10 (Downward-sloping left wing). Assume that $F_{V}$ is a continuous function. Suppose that there exist $\alpha \in(0,1)$ and $\beta>0$ with $\frac{1}{\alpha}-\frac{1}{\beta}=1$ such that $F_{V}$ satisfies (3.12) with $s<0$ or equivalently $\mathcal{L}_{V}$ satisfies (3.13) with $r<0$. Then the left-wing index $\tilde{q}_{V}=\infty$ and $I(K) \rightarrow 0$ as $K \rightarrow 0$.

Proof. Since (3.12) holds with $s<0$, it is possible to find $c>0$ and $x_{0}>0$ s.t. $F_{V}(x) \leq$ $e^{-c x^{-\beta}}$ for $x \geq x_{0}$. As $V_{T}$ has a positive distribution, for a given $q>0$ we can proceed as follows

$$
\begin{aligned}
\mathbb{E}\left[V_{T}^{-q}\right] & =\int_{0}^{+\infty} \mathbb{Q}\left(V_{T}^{-q}>x\right) d x \leq x_{0}+\int_{x_{0}}^{\infty} F_{V}\left(x^{-1 / q}\right) d x \\
& \leq x_{0}+\int_{0}^{x_{0}^{-1 / q}} F_{V}(x) q x^{-q-1} d x \leq x_{0}+\int_{0}^{x_{0}^{-1 / q}} e^{-c x^{-\beta}} q x^{-q-1} d x<\infty .
\end{aligned}
$$

So $\tilde{q}_{V}=\infty$ and Gulisashvili's criterion (3.6) applies. Notice that, for $K$ small enough, the following holds

$$
P(K)=\int_{0}^{K} F_{V}(x) d x \leq \int_{1 / K}^{+\infty} x^{-2} e^{-c x^{\beta}} d x \leq K e^{-c K^{-\beta}},
$$

where the last inequality follows by applying the property (3.9) to the rapidly varying function $f(x)=e^{-c x^{\beta}}$. The result follows now immediately from (3.6).

\subsection{Ornstein-Uhlenbeck Specifications}

We now proceed to the analysis of the volatility of variance in the SVJ-v model. The realized variance $V_{T}$ inherits the distributional properties of the jump process $J$ in an 
essentially unaltered manner. We consider the non-Gaussian Ornstein-Uhlenbeck (OU) specification which is obtained by setting $\eta=\varepsilon=0$ in the full SVJ-v specification (3.1). Hence, the instantaneous variance $v$ moves uniquely by jumps according to the following dynamics

$$
d v_{t}=-\lambda v_{t} d t+d J_{t}
$$

Stochastic volatility models of the OU type were first proposed by Barndorff-Nielsen and Shephard (2001) and have since then been studied extensively (see, e.g., Barndorff-Nielsen and Shephard (2003), Nicolato and Venardos (2003)). Besides possessing the capability of capturing stylized features of financial time series, OU type processes are extremely tractable from a mathematical viewpoint. For example, it is immediate to verify that the instantaneous variance process is given by

$$
v_{t}=e^{-\lambda t} v_{0}+\int_{0}^{t} e^{-\lambda(t-s)} d J_{s} .
$$

A further integration yields the following explicit expression for the annualized realized variance

$$
V_{T}=\frac{1}{T} \int_{0}^{T} v_{t} d t=v_{0} \epsilon(0, T)+\int_{0}^{T} \epsilon(t, T) d J_{t},
$$

where we have employed the compact notation

$$
\epsilon(t, T)=\frac{1-e^{-\lambda(T-t)}}{\lambda T} .
$$

Since $\epsilon(t, T)$ is a positive and decreasing function, we see immediately from expression (3.15) that for any $0<T_{1}<T$ the realized variance $V_{T}$ satisfies the following bounds

$$
v_{0} M+m J_{T_{1}}<V_{T}<v_{0} M+M J_{T}
$$

where $m$ and $M$ are positive constants given by $m=\epsilon\left(T_{1}, T\right)$ and $M=\epsilon(0, T)$. The corresponding Laplace transform of realized variance takes the explicit form

$$
\mathcal{L}_{V}(u)=\exp \left(-u \epsilon(0, T) v_{0}+\int_{0}^{T} \kappa_{J}(u \epsilon(t, T)) d t\right)
$$

where $\kappa_{J}$ is the cumulant function of $J_{1}$ as defined in (3.2).

For further details on OU type processes as well as the original derivation of the expressions reported above, we refer to Barndorff-Nielsen and Shephard (2001). A less well-known result yields the explicit calculation of the Lévy measure $\nu_{V}(d x)$ of the realized 
variance in terms of the Lévy measure $\nu_{J}(d x)$ of the driving subordinator $J$. Assume for simplicity that $\nu_{J}(d x)$ is absolutely continuous, and let $n_{J}$ denote its density. Then, Barndorff-Nielsen and Shephard (2003) show that also $\nu_{V}(d x)$ is absolutely continuous with density $n_{V}$ given by

$$
n_{V}(x)=\int_{x / \epsilon(0, T)}^{\infty} n_{J}(y) \frac{T}{y-\lambda T x} d y
$$

Let us now look at the jump impact on the asymptotic behavior of the implied volatility of variance for large strikes. Consistent with the adopted notation, the distribution function, the distribution tail, and the Laplace transform of the unit time law $J_{1}$ will be denoted by

$$
F_{J}(x)=\mathbb{Q}\left(J_{1} \leq x\right), \quad \bar{F}_{J}(x)=1-F_{J}(x), \quad \mathcal{L}_{J}(x)=\mathbb{E}\left[e^{-x J_{1}}\right]=\exp \kappa_{J}(x) .
$$

Our first result states that the realized variance $V_{T}$ has the same number of finite positive moments of the unit time law $J_{1}$. Moreover, if $J_{1}$ has a regularly varying tail, this property is transferred to the realized variance and the index of variation is maintained.

Proposition 11 (OU upward-sloping right wing). The following statements hold:

(i) Let $\tilde{p}_{J}=\sup \left\{p: \mathbb{E}\left[J_{1}^{p+1}\right]<\infty\right\}$. Then Lee's formula (3.3) for large strikes holds with index $\tilde{p}_{V}=\tilde{p}_{J}$.

(ii) Assume furthermore that $F_{J}$ is absolutely continuous with density $f_{J} \in R_{-\alpha-2}$ for $\alpha>0$. Then also $F_{J}$ is absolutely continuous with density $f_{V} \in R_{-\alpha-2}$. In this case the right-wing index is given by $\tilde{p}_{V}=\alpha$.

Proof. The chain of inequalities (3.17) implies that for any $p \geq 0$

$$
\mathbb{E}\left[\left(v_{0} M+m J_{T_{1}}\right)^{p}\right] \leq \mathbb{E}\left[V_{T}^{p}\right] \leq \mathbb{E}\left[\left(v_{0} M+M J_{T}\right)^{p}\right] .
$$

Statement $(i)$ now follows by recalling that the finiteness of moments of a Lévy process is a time independent property. See Theorem 25.3, p. 159 in Sato (1999). As for statement (ii), it follows from Remark 25.14., p. 164 in Sato (1999) that an infinitely divisible distribution is regularly varying if and only if its Lévy density is regularly varying with the same index. Hence, to conclude that $f_{V} \in R_{-\alpha-2}$, it suffices to show that $\nu_{V} \in R_{-\alpha-2}$. Since $\nu_{J} \in R_{-\alpha-2}$, from representation (3.8) it follows that $\nu_{V}$ given in (3.19) takes the form

$$
\nu_{V}(x)=\int_{x / \epsilon(0, T)}^{\infty} \ell(y) \frac{y^{-\alpha-2}}{(y-\lambda T x)} d y
$$


where $\ell \in R_{0}$. For $t>0$ the change of variable $z:=y / t$ yields

$$
\nu_{V}(t x)=t^{-\alpha-2} \int_{x / \epsilon(0, T)}^{\infty} \ell(t z) \frac{z^{-\alpha-2}}{(z-\lambda T x)} d z
$$

As $\ell \in R_{0}$ then for a given $t>0$ and $\varepsilon>0$ we can find $z_{*}$ s.t.

$$
(1-\varepsilon) \ell(z) \frac{z^{-\alpha-2}}{(z-\lambda T x)} \leq \ell(t z) \frac{z^{-\alpha-2}}{(z-\lambda T x)} \leq(1+\varepsilon) \ell(z) \frac{z^{-\alpha-2}}{(z-\lambda T x)}
$$

holds for all $z \geq z_{*}$. Since $\varepsilon$ is arbitrary we conclude that

$$
\frac{\nu_{V}(t x)}{\nu_{V}(x)} \rightarrow t^{-\alpha-2} \quad \text { as } \quad x \rightarrow \infty
$$

Finally $\tilde{p}_{V}=\alpha$ since $f_{V} \in R_{-\alpha-2}$ implies that $\bar{F}_{V} \in R_{-\alpha-1}$ and in virtue of Proposition 7 .

Next, we provide sufficient conditions for $J_{1}$ to guarantee a right wing which for large strikes is downward-sloping and vanishing to zero. In essence, we require that the tail of $J_{1}$ is either thin or Weibull equivalent.

Proposition 12 (OU downward-sloping right wing). Let $\alpha, \beta, \gamma>0$, and suppose that one of following statements holds.

a. The tail condition

$$
\bar{F}_{J}(x) \leq \gamma e^{-\alpha x^{\beta}} \quad \text { for } \quad x \in\left(x_{0}, \infty\right)
$$

is satisfied with $\beta \geq 1$.

b. The tail condition

$$
\bar{F}_{J}(x) \sim \gamma e^{-\alpha x^{\beta}} \quad \text { as } \quad x \rightarrow \infty
$$

is satisfied with $0<\beta<1$.

Then $\tilde{p}_{V}=\infty$ and $I(K) \rightarrow 0$ as $K \rightarrow \infty$

Proof. In either case, the distribution tail of $J_{1}$ is dominated by a Weibull type tail. Hence, $\tilde{p}_{V}=\tilde{p}_{J}=\infty$ in virtue of Proposition 8. Suppose that the thin-tail condition (3.20) is satisfied. The equivalence (3.11) implies that zero lies in the interior $\stackrel{\mathcal{D}}{J}_{J}$ of the Laplace transform $\mathcal{L}_{J}(u)$ of the unit time law $J_{1}$. Then we see from expression (3.18) 
that $0 \in \stackrel{\circ}{\mathcal{D}}_{V}$ as well and therefore the statement holds. Assume now that $J_{1}$ is tailequivalent to a Weibull distribution with $0<\beta<1$ as stated in (3.21). Then $J_{1}$ is a sub-exponential ${ }^{1}$ distribution and in virtue of Remark 25.14, p.164 in Sato (1999) the following tail equivalence

$$
\mathbb{Q}\left(J_{t} \geq x\right) \sim t \nu_{J}(x, \infty) \sim t \bar{F}_{J}(x) \sim t \gamma e^{-\alpha x^{\beta}}
$$

holds at any time $t>0$ and in particular at $t=T$. Then the upper bound in (3.17) implies that there exist positive constants $\hat{\alpha}$ and $\hat{\gamma}$ such that for $x$ large enough it holds that

$$
\bar{F}_{V}(x)=\mathbb{Q}\left(V_{T} \geq x\right) \leq \mathbb{Q}\left(J_{T} \geq \frac{x-v_{0}}{M}\right) \leq \hat{\gamma} e^{-\hat{\alpha} x^{\beta}}
$$

and therefore the statement follows from Proposition 10.

Let us now analyze the volatility of variance behavior for small strikes. First, we notice from expression (3.15) that in models of the OU type, the realized variance $V_{T}$ is bounded from below by $v_{0} \epsilon(0, T)$. As a consequence, unless $v_{0}=0$, the implied volatility $I(K)$ is not well-defined for small $K$ rendering the investigation of asymptotic properties of the left wing a nonsensical task. Therefore, we will carry out the remaining part of our asymptotic analysis of the OU model under the assumption that $v_{0}=0$.

Once again, we start by providing sufficient conditions on the jump distribution $J_{1}$ for the left wing of the volatility of variance to be upward-sloping to infinity for small strikes, i.e. $I(K) \rightarrow \infty$ as $K \rightarrow 0$. Assume that $F_{V}$ is a continuous function. Suppose that for an $\alpha \geq 0, \bar{F}_{V} \in R_{-\alpha}$ or equivalently $\mathcal{L}_{V}(x) \in R_{-\alpha}$. Then Lee's formula (3.4) for small strikes holds with index $\tilde{q}_{V}=\alpha$.

Proposition 13 (OU upward-sloping left wing). Assume $F_{J}$ is continuous. Suppose that for an $\alpha \geq 0, F_{J}(1 / x) \in R_{-\alpha}$ or equivalently $\mathcal{L}_{J}(x) \in R_{-\alpha}$. Then Lee's formula (3.4) for small strikes holds with index $\tilde{q}_{V}=\alpha T$.

Proof. Recall that for $v_{0}=0$ the Laplace transform becomes $\mathcal{L}_{V}(x)=\exp \int_{0}^{T} \kappa_{J}(x \epsilon(s, T)) d s$. Applying the change of variable $z:=\epsilon(t, T)$ we have

$$
\lim _{x \rightarrow \infty} \frac{\mathcal{L}_{V}(t x)}{\mathcal{L}_{V}(x)}=\lim _{x \rightarrow \infty} \exp \int_{0}^{\epsilon(0, T)} \frac{T}{1-\lambda T z}\left(\kappa_{J}(t x z)-\kappa_{J}(x z)\right) d z .
$$

\footnotetext{
${ }^{1} \mathrm{~A}$ distribution $F$ on $\mathbb{R}_{+}$is called sub-exponential if $\lim _{x \rightarrow \infty} \frac{\overline{F^{n *}}(x)}{\bar{F}(x)}=n$, for $n \geq 2$, where $F^{n *}$ denotes the $n$th convolution.
} 
Moreover, from boundary (2.6) p. 180 in Korevaar (2004) we can apply a dominated convergence argument to interchange the limit and integral, thus one obtains

$$
\lim _{x \rightarrow \infty} \frac{\mathcal{L}_{V}(t x)}{\mathcal{L}_{V}(x)}=\exp \int_{0}^{\epsilon(0, T)} \frac{T}{1-\lambda T z} \lim _{x \rightarrow \infty}\left(\log \left(\mathcal{L}_{J}(t x z) / \mathcal{L}_{J}(x z)\right)\right) d z=t^{-\alpha T} .
$$

Therefore, $\mathcal{L}_{V} \in R_{-\alpha T}$ and the statement follows from Proposition 9.

We conclude with a sufficient condition on the jump distribution for the left wing to be downward-sloping towards zero.

Proposition 14 (OU downward-sloping left wing). Assume that $F_{J}$ is a continuous function. Suppose that there exist $\alpha \in(0,1)$ and $\beta>0$ with $\frac{1}{\alpha}-\frac{1}{\beta}=1$ such that $F_{J}$ satisfies (3.12) with $s<0$ or equivalently $\mathcal{L}_{J}$ satisfies (3.13) with $r<0$. Then the index $\tilde{q}_{V}=\infty$ and $I(K) \rightarrow 0$ as $K \rightarrow 0$.

Proof. As in proof of Proposition 9, we make the change of variable $z:=\epsilon(t, T)$ and exchange limit and integration to obtain

$$
\begin{aligned}
\lim _{x \rightarrow \infty} \frac{\log \mathcal{L}_{V}(x)}{x^{\alpha}} & =T \int_{0}^{\epsilon(0, T)} \lim _{x \rightarrow \infty} \frac{\kappa_{J}(z x)}{(z x)^{\alpha}} \frac{z^{\alpha}}{1-\lambda T z} d z= \\
& =r T \int_{0}^{\epsilon(0, T)} \frac{z^{\alpha}}{1-\lambda T z} d z=\bar{r}<0 .
\end{aligned}
$$

Once again, interchanging integral and limit is possible due to a dominated convergence argument following from boundary (2.6) p. 180 in Korevaar (2004). Therefore, $\mathcal{L}_{V}$ satisfies condition (3.13) with $\bar{r}<0$ and the results follows now from Proposition 10.

To sum up, we have provided sufficient conditions for the wing asymptotics of the variance smile in the OU specification. In fact, we saw that the distributional properties of the jump process $J$ were transferred basically unchanged to the distribution of the realized variance $V_{T}$. Next, we take a closer look at some specific distributional choices for the jumps.

\subsection{A Menagerie of Positive Distributions}

In this section we shortly recapitulate a number of positive distributions and some of their key characteristics. In parallel, we analyze the asymptotic behavior of the volatility of variance in the OU specification for particular distributions of the jumps $J$. These 
results are summarized in Table 3.1. Finally, we provide numerical examples for the OU specification with Gamma, Inverse Gamma, and Inverse Gaussian jumps, respectively.

Gamma Distribution As first case consider a $\Gamma(\alpha, \beta)$ distribution with density function

$$
f_{\Gamma}(x)=\frac{\beta^{\alpha}}{\Gamma(\alpha)} x^{\alpha-1} e^{-\beta x} \mathbf{1}_{x \geq 0}
$$

and Laplace transform

$$
\mathcal{L}_{\Gamma}(u)=\left(1+\frac{u}{\beta}\right)^{-\alpha}, \quad u>-\beta .
$$

$\mathcal{L}_{\Gamma}$ is a regularly varying function of index $-\alpha$ at infinity since

$$
\lim _{u \rightarrow \infty} \frac{\mathcal{L}_{\Gamma}(t u)}{\mathcal{L}_{\Gamma}(u)}=\lim _{u \rightarrow \infty}\left(\frac{1+t u / \beta}{1+u / \beta}\right)^{-\alpha}=t^{-\alpha} .
$$

As a special case of the Gamma distribution, we obtain the exponential distribution when $\alpha=1$. Let us consider the OU specification. If we choose $J_{1} \sim \Gamma(\alpha, \beta)$ then the right wing is downward-sloping since $0 \in \stackrel{\circ}{\mathcal{D}}_{J}$. In addition, Proposition 13 is satisfied and the left wing is upward-sloping.

Inverse Gamma Distribution Consider a $I \Gamma(\nu, \mu)$ distribution with density function

$$
f_{I \Gamma}(x)=\frac{\mu^{\nu}}{\Gamma(\nu)} x^{-\nu-1} e^{-\mu / x} \mathbf{1}_{x \geq 0}
$$

and Laplace transform

$$
\mathcal{L}_{I \Gamma}(u)=\frac{2(\mu u)^{\nu / 2}}{\Gamma(\nu)} K_{\nu}(\sqrt{4 \mu u})
$$

where $K_{\nu}$ is the modified Bessel function of the second kind. Using the fact that for large arguments $K_{\nu}(z) \sim \sqrt{\frac{\pi}{2 z}} e^{-z}$ (see Abramowitz and Stegun (1987) p. 378), we obtain

$$
\lim _{u \rightarrow \infty} \frac{\log \mathcal{L}_{I \Gamma}(u)}{u^{1 / 2}}=\lim _{u \rightarrow \infty} \frac{\nu \log (\mu u)+\log \left(K_{\nu}(\sqrt{4 \mu u})\right)-\log \Gamma(\nu)}{u^{1 / 2}}=-\sqrt{4 \mu}<0 .
$$

Consider the OU specification. If we choose $J_{1} \sim I \Gamma(\nu, \mu)$ then $\tilde{p}_{J}<\infty$ and the right wing is upward-sloping. Moreover, Proposition 14 holds and the left wing is downward-sloping. 
Generalized Inverse Gaussian Distribution Consider a $G I G(\lambda, a, b)$ distribution with density

$$
f_{G I G}(x)=\frac{(b / a)^{\lambda}}{2 K_{\lambda}(a b)} x^{\lambda-1} \exp \left(-\frac{1}{2}\left(a^{2} x^{-1}+b^{2} x\right)\right)
$$

and Laplace transform

$$
\mathcal{L}_{G I G}(u)=\left(\frac{b^{2}}{b^{2}+2 u}\right)^{\lambda / 2} \frac{K_{\lambda}\left(\sqrt{a^{2}\left(b^{2}+2 u\right)}\right)}{K_{\lambda}(a b)} .
$$

It is easy to verify that

$$
\lim _{u \rightarrow \infty} \frac{\log \mathcal{L}_{G I G}(u)}{u^{\frac{1}{2}}}<0 .
$$

As a particular instance of the GIG distribution we consider the case $\lambda=-\frac{1}{2}$ which gives us the Inverse Gaussian distribution. In the OU specification, if we choose $J_{1} \sim G I G(\lambda, a, b)$ then $0 \in \stackrel{\circ}{\mathcal{D}}_{J}$ and therefore the right wing is downward-sloping. Also, Proposition 14 is satisfied and the left wing is downward-sloping.

Tempered Stable Distribution The $T S(k, a, b)$ distribution is an infinitely-divisible distribution characterized by the following Lévy measure

$$
\nu_{T S}(d x)=a 2^{k} \frac{k}{\Gamma(1-k)} x^{-k-1} \exp \left(-\frac{1}{2} b^{1 / k} x\right) \mathbf{1}_{(x>0)} d x
$$

where $a>0, b \geq 0,0<k<1$. The corresponding Laplace transform is

$$
\mathcal{L}_{T S}(u)=\exp \left(a b-a\left(b^{1 / k}+2 u\right)^{k}\right)
$$

which satisfies

$$
\lim _{u \rightarrow \infty} \frac{\log \mathcal{L}_{T S}(u)}{u^{k}}<0 .
$$

If we consider the OU specification with $J_{1} \sim T S(k, a, b)$, then $0 \in \stackrel{\circ}{\mathcal{D}}_{J}$ and the right wing is downward-sloping. For the left wing, Proposition 14 implies a downward-sloping skew.

Weibull Distribution Consider a $W(c, \beta)$ distribution with density

$$
f_{W}(x)=\frac{c x^{c-1}}{\beta} \exp \left(\frac{-x^{c}}{\beta}\right)
$$

and survival function

$$
\bar{F}_{W}(x)=e^{-(x / \beta)^{c}} .
$$


It can be shown that a Weibull random variable has just $c$ negative moments. Deriving an explicit expression for the Laplace transform of a Weibull r.v. is a topic of ongoing research, see for instance Nadarajah and Kotz (2007).

Consider again the OU specification and let $J_{1} \sim W(c, \beta)$. Condition (b) in Proposition 12 holds and the right wing is downward-sloping. In addition, from Proposition 13 we have that the left wing is upward-sloping.

Table 3.1: Asymptotic behavior of implied volatility of variance for the $O U$ specification

\begin{tabular}{lcc}
\hline \hline & \multicolumn{2}{c}{ Wing asymptotics } \\
\cline { 2 - 3 } Jump distribution & Small strikes & Large strikes \\
\hline Gamma & Upward & Downward \\
Exponential & Upward & Downward \\
Inverse Gamma & Downward & Upward \\
Inverse Gaussian & Downward & Downward \\
Generalized Inverse Gaussian & Downward & Downward \\
Tempered Stable & Downward & Downward \\
Weibull & Upward & Downward \\
\hline \hline
\end{tabular}

\subsubsection{Numerical Examples}

We illustrate the impact of the distribution of jumps in the OU specification by numerical examples. Specifically, we consider Gamma, Inverse Gamma, and Inverse Gaussian jumps. The variance call price $C(K)$ and the associated implied volatility $I(K)$ can be computed by means of transform methods whenever the Laplace transform of the realized variance $\mathcal{L}_{V}$ is available in closed-form. In fact, Carr et al. (2005) show that the Laplace transform $\mathcal{L}_{C}$ of the call price $C(K)$ can be expressed as

$$
\mathcal{L}_{C}(u)=\int_{0}^{\infty} e^{-u K} C(K) d K=\frac{\mathcal{L}_{V}(u)-1}{u^{2}}+\frac{V_{0}}{u}
$$

Applying a Laplace inversion algorithm to (3.22) allows us to obtain prices of options on realized variance for a sequence of variance strikes. Several numerical Laplace inversion algorithms have been proposed in the literature. In particular, we refer to the works of Weeks (1966), Dubner and Abate (1968), Stehfest (1970), Crump (1976), Abate and Whitt 
(1995), and Iseger (2006), while the recent study of Drimus (2012) considers applications specific to realized variance options.

Figure 3.1 plots implied volatilities against moneyness when the jumps are Gamma, Inverse-Gamma and IG distributed. We define moneyness as the variance strike $K$ divided by the mean realized variance $V_{0}$ and at-the-money options are striked at $K=V_{0}$. We consider a maturity of three months and we obtain the parameters of the different jump distributions by matching their mean and variance. In particular, we take $\alpha=18$ and $\beta=22.8$ for the Gamma specification, $\nu=20$ and $\mu=15$ for the Inverse-Gamma and $a=3.7697$ and $b=4.7749$ for the IG distribution. The value of $\lambda$ is the same in all cases and it is equal to 8 . The plots confirm that when the jumps are Gamma distributed, the volatility surface for realized variance options is downward-sloping, clearly at odds with the upward-sloping smile observed in variance markets. In contrast, the Inverse Gamma distribution makes the volatility surface of realized variance upward-sloping and finally, for the Inverse-Gaussian jumps we observe a frown.

To further support these observations, we investigate in Figures 3.2-3.4 the sensitivity of the variance implied volatility with respect to the parameters of the jump distribution. To disclose the ceteris paribus effects on the implied volatility, we change one parameter while keeping any remaining parameters fixed. For each parameter set, we plot the implied volatility curve against variance strikes. For the selected jump distributions, we make the common observation that while altering the parameters of the distribution changes the levels of implied volatility and the wideness of the smile in variance strikes, the shapes of the implied volatility curves persist across different parameter values.

\subsection{Full SVJ-v Specifications}

The Heston stochastic volatility model constitute a popular choice in the equity markets. Yet, as documented in Drimus (2012), the model leads to a downward-sloping skew for the volatility of variance. Several authors have tried to amend this shortcoming by augmenting the Heston model with compound Poisson jumps having exponential jump sizes (see Sepp (2008a), Sepp (2008b), and Lian and Zhu (2011)). In this final section we consider full specifications of the SVJ-v model in (3.1), which includes both the Heston model and the Heston model with exponential jumps as special cases. Recall that the instantaneous 

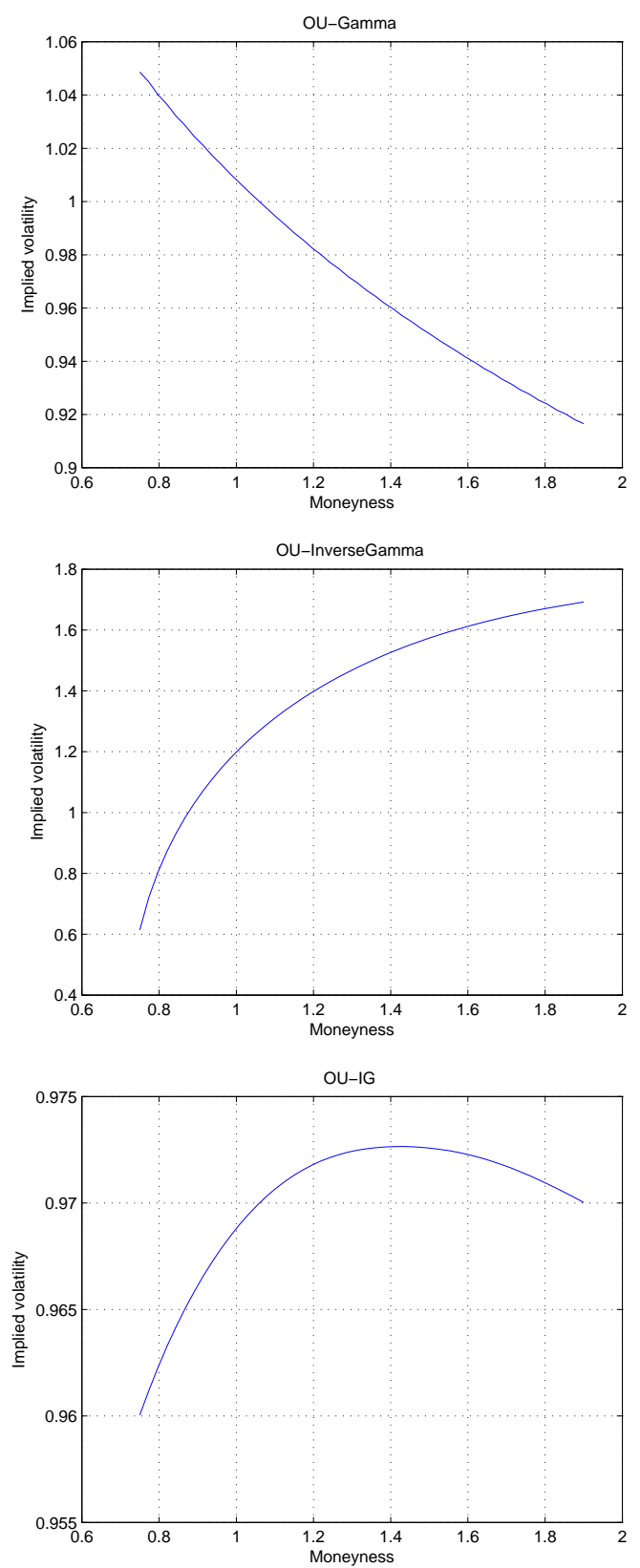

Figure 3.1: Implied volatilities of variance for the OU-Gamma with parameters $\alpha=18$, $\beta=22.8$ (top), OU-InverseGamma with $\nu=20, \mu=15$ (middle), and OU-IG with $a=3.7697, b=4.7749$ (bottom). In all cases we take $\lambda=8$, and $v_{0}=0$ and we obtain the parameters of the different jump specifications by matching the mean and the variance.

variance is given by the CIR dynamics augmented with jumps

$$
d v_{t}=\lambda\left(\eta-v_{t}\right) d t+\epsilon \sqrt{v_{t}} d B_{t}+d J_{t} .
$$




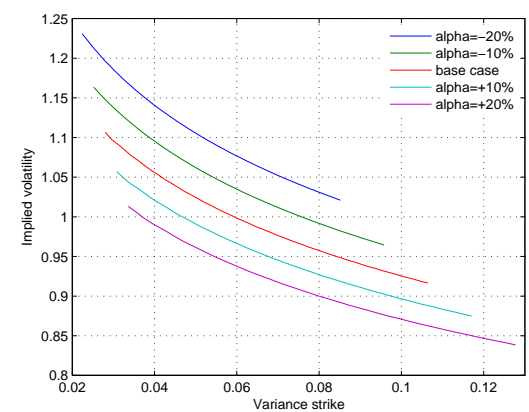

(a) Changes in $\alpha$

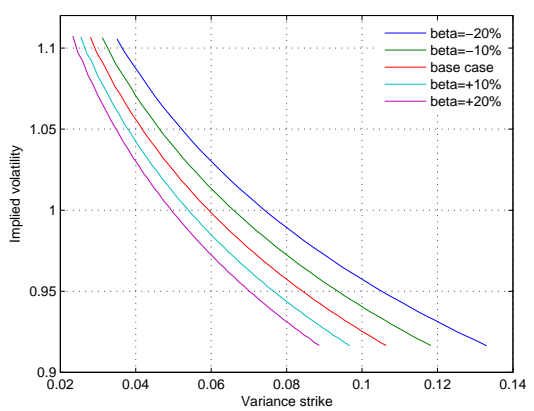

(b) Changes in $\beta$

Figure 3.2: OU-Gamma parameter sensitivities. Base case parameters: $\alpha=18, \beta=22.8$, $\lambda=8$, and $v_{0}=0$.

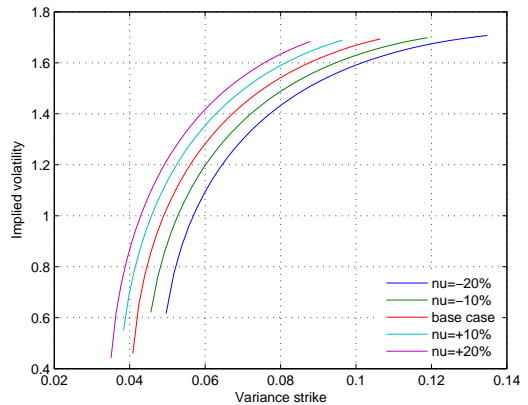

(a) Changes in $\nu$

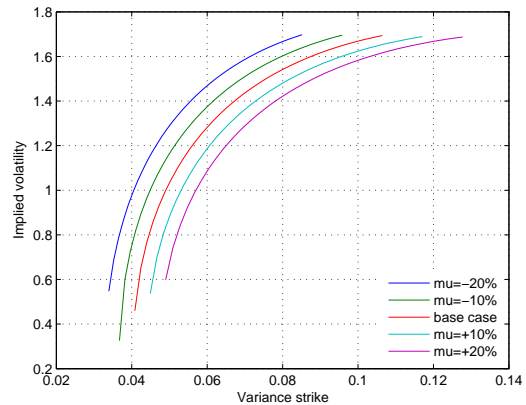

(b) Changes in $\mu$

Figure 3.3: OU-Inverse Gamma parameter sensitivities. Base case parameters: $\nu=20$, $\mu=15, \lambda=8$, and $v_{0}=0$.

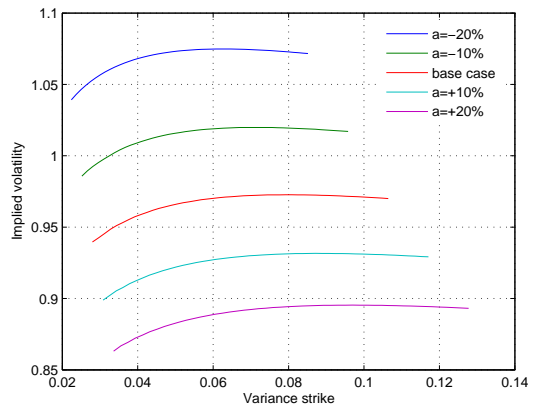

(a) Changes in $a$

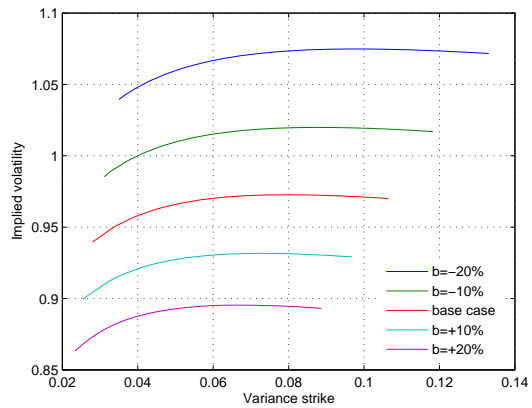

(b) Changes in $b$

Figure 3.4: $O U-I G$ parameter sensitivities. Base case parameters: $a=3.7697, b=4.7749$, $\lambda=12$, and $v_{0}=0$. 
Here, we specify the jump process $J$ as a compound Poisson process with intensity $l$ and jump size distribution $Z$, i.e.

$$
J_{t}=\sum_{i=1}^{N(t)} Z_{i}, \quad Z_{i} \sim \text { i.i.d. } Z
$$

with $N(t)$ being a Poisson process with intensity $l$. In this case the cumulant exponent $\kappa_{J}$ is given by

$$
\kappa_{J}(x)=l\left(\mathcal{L}_{Z}(x)-1\right)
$$

where $\mathcal{L}_{Z}$ denotes the Laplace transform of the jump size.

From Duffie et al. (2000) the Laplace transform of the realized variance is available in closed-form

$$
\mathcal{L}_{V}(u)=\mathbb{E}\left[e^{-u V_{T}}\right]=e^{\alpha(u, T)+\beta(u, T) v_{0}}
$$

with functions $\alpha(u, T)$ and $\beta(u, T)$ given by

$$
\begin{aligned}
& \beta(u, T)=\frac{1-e^{-\gamma T}}{c_{1}+d_{1} e^{-\gamma T}} \\
& \alpha(u, T)=-\frac{2 \lambda \eta}{\epsilon^{2}} \log \frac{c_{1}+d_{1} e^{-\gamma T}}{c_{1}+d_{1}}+\frac{\lambda \eta T}{c_{1}}-\int_{0}^{T} \kappa_{J}(-\beta(u, s)) d s
\end{aligned}
$$

where $\kappa_{J}(\cdot)$ is the cumulant exponent (3.2) of the jump process $J$, while $c_{1}=\frac{\lambda+\gamma}{-2 u / T}$, $d_{1}=\frac{\lambda-\gamma}{2 u / T}$ and $\gamma=\sqrt{\lambda^{2}+2 \varepsilon^{2} u / T}$.

Analogous to the simpler OU models, we provide now results on the impact of the jump distribution for specifications of the full SVJ-v model. First, we consider the right wing asymptotics. Once again, we link the number of finite positive moments of the jumps with those of the realized variance. We are, however, not able to attain the same level of asymptotic precision as in the OU case. Nevertheless, we provide bounds for the Lee index $\tilde{p}_{V}$ which are sufficient for describing the qualitative asymptotic behavior of the right wing.

Proposition 15 (SVJ-v upward-sloping right wing). The following statements hold

(i) Let $\tilde{p}_{J}$ be defined as in Proposition (11). Then Lee's formula (3.3) for large strikes holds with $\left\lfloor\tilde{p}_{J}\right\rfloor \leq \tilde{p}_{V} \leq\left\lfloor\tilde{p}_{J}\right\rfloor+1$ (with $\tilde{p}_{V}=\infty$ if $\tilde{p}_{J}=\infty$ ).

(ii) If furthermore $\bar{F}_{J} \in R_{-\alpha-1}$ then $\lfloor\alpha\rfloor \leq \tilde{p}_{V} \leq\lfloor\alpha\rfloor+1$. 
Proof. Statement (i) follows from classical results of probability theory linking the number of integer moments of a nonnegative random variable $X$ to the regularity of its Laplace transform $\mathcal{L}_{X}$ at zero. Specifically, $X$ has a finite $n$th moment if and only if the $n$th derivative $\mathcal{L}_{X}^{(n)}$ has finite limit at zero (see Feller (1974)). The result then follows from observing that $\mathcal{L}_{V}(u)$ is differentiable if and only if the Laplace transform of the jump distribution is differentiable. Statement (ii) is a consequence of the definition of regular variation.

Proposition 16 (SVJ-v downward-sloping right wing). If the jump distribution in (3.23) has a thin tail, i.e.

$$
\bar{F}_{J}(x) \leq \gamma e^{-\alpha x^{\beta}} \quad \text { for } \quad x \in\left(x_{0}, \infty\right)
$$

with $\beta \geq 1$, then $\tilde{p}_{V}=\infty$ and $I(K) \rightarrow 0$ as $K \rightarrow \infty$.

Proof. From equivalence (3.11) $0 \in \mathcal{D}_{J}$. A simple inspection of (3.26) shows that also $0 \in \mathcal{D}_{V}^{\circ}$ implying that $V_{T}$ has a thin tail. The result then follows from Proposition 8.

In the full SVJ-v specifications considered here, jumps have no effect on the asymptotic behavior of the left wing of the realized variance smile.

Proposition 17 (SVJ-v downward-sloping left wing). For any jump distribution in (3.23) it holds $\tilde{q}_{V}=\infty$ and $\lim _{K \rightarrow 0} I(K)=0$.

Proof. Recall the expression of the Laplace transform in (3.26) and observe that independent of the particular choice of the jump size distribution $Z$,

$$
\int_{0}^{T} \mathcal{L}_{Z}(-\beta(u, s)) d s \rightarrow 0 \quad \text { as } \quad u \rightarrow \infty
$$

We then obtain

$$
\lim _{u \rightarrow \infty} \frac{\log \mathcal{L}_{V}(u)}{u^{-1 / 2}} \leq 0
$$

and the result follows from Proposition 10.

Besides the mandatory benchmark of the Heston model, we consider in the sequel two genuine specifications of the SVJ-v model allowing for both jumps as well as the diffusion component. Specifically, we let the jump size distribution $Z$ be exponential and Inverse Gamma. In the Heston model and the SVJ-v model with exponential jumps the realized variance $V_{T}$ has a thin tail and from Proposition 16 it follows that the right wing 


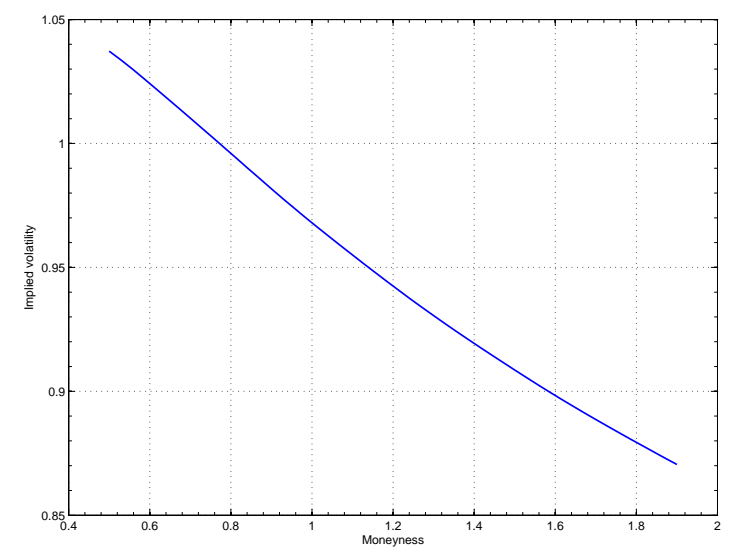

Figure 3.5: Implied volatilities of variance for the Heston model.
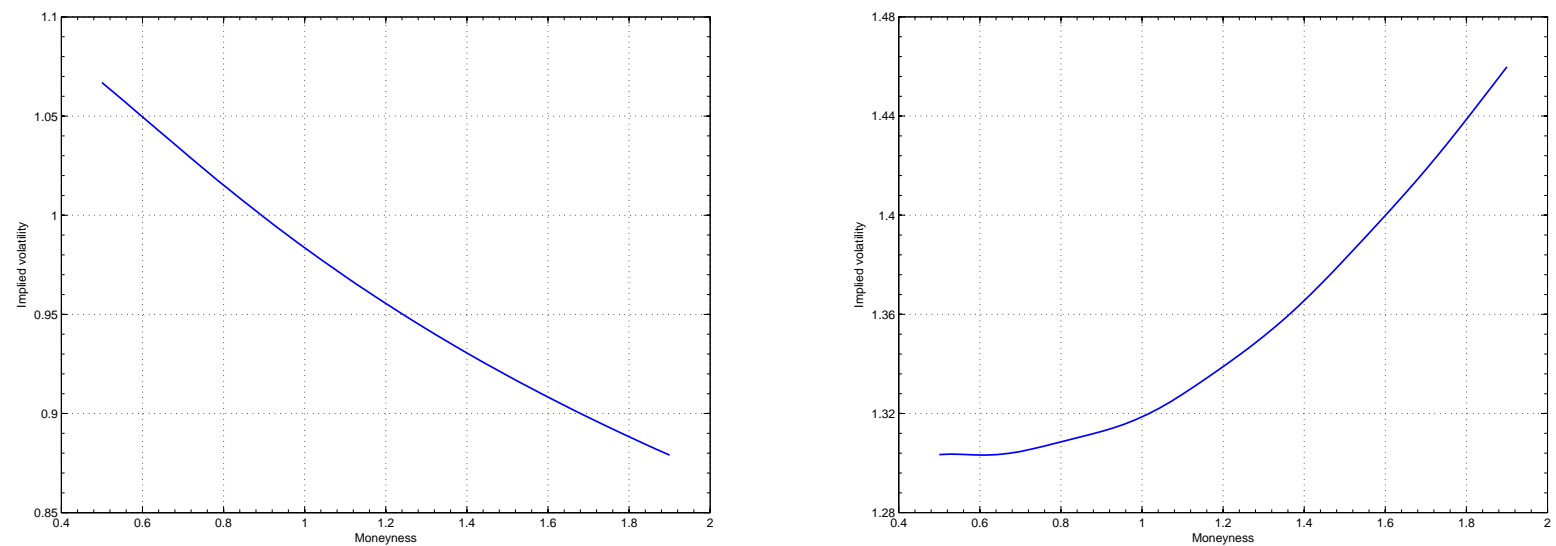

Figure 3.6: Implied volatilities of variance for the SVJ-v model with exponential jumps (left) and Inverse Gamma jumps (right). 
is downward-sloping. In contrast, for the SVJ-v model with Inverse Gamma jumps, the right wing is upward-sloping as a result of Proposition 15.

We now provide numerical examples for the three model cases. We consider 6-months options and we use the Heston parameters from Bakshi et al. (1997) $v_{0}=0.0348, \kappa=1.15$, $\theta=0.0348, \epsilon=0.39$ obtained by calibration to out-of-the-money options on the S\&P500 index. Figure 3.5 plots the implied volatilities of variance for the Heston model against moneyness. One observes that when using parameters fitted to equity option quotes, the obtained implied volatility skew is downward-sloping. This shows that the domain of validity of the left wing asymptotics can be rather narrow.

Next, we augment the Heston model with exponential jump sizes $Z \sim \Gamma(1, \beta)$ with intensity $l=1.5$ and mean jump size $1 / \beta=0.1^{2}$. The resulting implied volatilities of variance are shown in Figure 3.6. Once again, we obtain a downward-sloping skew. However, if we substitute the jump size distribution $Z$ with an Inverse Gamma law, we observe a dramatic change in the shape and level of the volatility of variance. Specifically, this leads to an upward-sloping skew as in Figure 3.6 where we have used Inverse Gamma jump parameters $\nu=3$ and $\mu=2 \times 0.1^{2}$ obtained by matching the first and the second moment of the jump size with those in the exponential case.

\subsection{Conclusion}

We have considered options on realized variance in a tractable affine stochastic volatility model that generalizes the seminal Heston (1993) by augmenting it with jumps in the instantaneous variance. The model allowed us to isolate the unique impact of the jump distribution and we have shown that it has a profound effect on the characteristics and shape of the implied volatility of variance smile. We provided sufficient conditions for the asymptotic behavior of the volatility of variance for small and large strikes. In particular, we showed that by selecting alternative jump distributions, one obtains fundamentally different shapes of the implied volatility smile. Some distributions of jumps predict implied volatilities of variance that are clearly at odds with the upward-sloping volatility skew observed in variance markets. 


\subsection{Appendix}

Proof of Theorem 4. The proof of this Theorem is the analogous of the proof of Theorem 1.26 p. 16 in Soulier (2009).

Part $(\mathrm{a}) \Rightarrow(\mathrm{b})$ :

From the definition of regular variation at infinity we have $\lim _{y \rightarrow \infty} \frac{\mathcal{L}_{f}(s y)}{\mathcal{L}_{f}(y)}=s^{-\alpha} \forall s>0$. Notice that $s^{-\alpha}$ is the Laplace transform of the function $g(x)=\left\{\begin{array}{ll}H(x) & \alpha=0 \\ \frac{x^{\alpha}}{\Gamma(\alpha)} & \alpha>0\end{array}\right.$ where $H(x)$ is the heavyside function defined as $H(x)=\left\{\begin{array}{ll}1 & x>0 \\ 0 & x=0\end{array}\right.$. On the other hand, $\frac{\mathcal{L}_{f}(s y)}{\mathcal{L}_{f}(y)}=$ $\int_{0}^{+\infty} e^{-s x} d g_{y}(x)$ where $g_{y}(x)=\frac{f(x / y)}{\mathcal{L}_{f}(y)}$. Combining the two equalities yields

$$
\lim _{y \rightarrow \infty} \int_{0}^{+\infty} e^{-s x} g_{y}(x) d x=\int_{0}^{+\infty} e^{-s x} g(x) d x
$$

which implies the pointwise convergence $g_{y}(s) \rightarrow_{y \rightarrow \infty} g(s)$. Therefore

$$
\frac{f(s / y)}{\mathcal{L}_{f}(y)} \rightarrow_{y \rightarrow \infty} \frac{s^{\alpha}}{\Gamma(1+\alpha)}
$$

and replacing $s=1$ we get statement (b) as well as (c).

Part $(\mathrm{b}) \Rightarrow(\mathrm{a})$ :

Let us denote $G(x)=f(1 / x)$. Integrating by parts in the definition of $\mathcal{L}_{f}(y)$ and performing the substitution $t=y u$ we get

$$
\frac{\mathcal{L}_{f}(y)}{f(1 / y)}=\int_{0}^{\infty} e^{-t} \frac{f(t / y)}{f(1 / y)} d t=\int_{0}^{+\infty} e^{-t} \frac{G\left(t^{-1} y\right)}{G(y)} d t
$$

Moreover, assuming that we can interchange the limit with the integral

$$
\lim _{y \rightarrow \infty} \frac{\mathcal{L}_{f}(y)}{G(y)}=\int_{0}^{\infty} e^{-t} \lim _{x \rightarrow \infty} \frac{G(y / t)}{G(y)}=\Gamma(\alpha+1) .
$$

So all we need in order to conclude the proof is some dominated convergence argument. We can proceed analyzing the two different cases $t \leq 1$ and $t>1$. In the first case, by recalling that $f(y)$ is a non-decreasing function, we have

$$
\frac{G(y / t)}{G(x)}=\frac{f(t / y)}{f(1 / y)} \leq 1
$$


whereas for $t>1$ we can use equation (2.6) p.180 in Korevaar (2004). Doing so we obtain the bounding $e^{-t} \frac{G\left(t^{-1} y\right)}{G(y)} \leq D(t)=\left\{\begin{array}{ll}e^{-t}, & t \leq 1 \\ \frac{1}{b} e^{-t} t^{\alpha+1}, & t>1\end{array}\right.$ with $\int_{0}^{\infty} D(t) d t<\infty$. 


\section{Chapter 4}

Consistent Valuation of Collateralized OTC

Deals under Counterparty Credit and

Funding Risk 


\title{
Consistent Valuation of Collateralized OTC Deals under Counterparty Credit and Funding Risk
}

\author{
D. Brigo ${ }^{\dagger} \quad$ Q. Liu $^{\dagger} \quad$ A. Pallavicini ${ }^{\S} \quad$ D. Sloth* \\ $\dagger$ Department of Mathematics, Imperial College, London \\ $\S$ Banca IMI, Milan \\ * Department of Economics and Business, Aarhus University, Aarhus
}

\begin{abstract}
We develop an arbitrage-free framework for consistent valuation of OTC derivative trades with collateralization, counterparty credit risk, and funding costs. Based on the risk-neutral pricing principle, we derive a general pricing equation where CVA, DVA, and FVA are introduced by simply modifying the payout cash-flows of the deal. Funding risk breaks the bilateral nature of the deal price and makes the pricing problem a highly non-linear and recursive one. This means that FVA is not generally an additive adjustment as commonly assumed by market participants. Our framework addresses common market practices of ISDA governed deals without restrictive assumptions on collateral margin payments and close-out netting rules. In particular, we allow for asymmetric collateral and funding rates. The pricing equation can be cast as a set of iterative equations that can be solved by leastsquares Monte Carlo and we propose such a simulation algorithm. Our numerical results confirm that funding risk has a non-trivial impact on the deal price.
\end{abstract}

\subsection{Introduction}

In the wake of the recent financial crisis, dealers and financial institutions have been forced to rethink how they value and hedge contingent claims traded in the over-the-counter (OTC) market. OTC derivatives are bilateral financial contracts negotiated between two 
default-risky entities. Yet, prior to the crisis, institutions tended to ignore the credit risk of high-quality rated counterparties, but as recent history has shown this was a particular dangerous assumption. Moreover, as banks became reluctant to lend to each other with the crisis rumbling through the Western economies, the spread between the rate on overnight indexed swaps (OISs) and the LIBOR rate blew up.

To keep up with this sudden change of game, dealers today make a number of adjustments when they book OTC trades. The credit valuation adjustment (CVA) corrects the price for the expected costs to the dealer due to the possibility that the counterparty may default, while the so-called debt valuation adjustment (DVA) is a correction for the expected benefits to the dealer due to his own default risk. The latter adjustment has the perverse effect that the dealer can book a profit as his default risk increases. Finally, dealers often adjust the price for the costs of funding the trade. In the industry, this practice is known as a funding valuation adjustment (FVA). When a derivatives desk executes a deal with a client, it backs the trade by hedging it with other dealers in the market. This involves borrowing or lending money and other assets. Classical derivatives pricing theory rests on the assumption that one can borrow and lend at a unique risk-free rate of interest. The seminal work of Black-Scholes-Merton showed that in this case an option can be replicated by a portfolio of equity and risk-free debt over any short period of time. Prior to the crisis, this assumption may have been reasonable with banks funding their hedging strategies at LIBOR. However, with drastically increasing spreads emerging as the crisis took hold, it became apparent that LIBOR is contaminated by credit risk and as such is an imperfect proxy of the risk-free rate.

In this paper we develop an arbitrage-free framework for consistent valuation of collateralized as well as uncollateralized OTC trades under counterparty credit and funding risk. The need to consistently account for the changed trading conditions in the valuation of derivatives is stressed by the sheer size of the OTC market. The market value of outstanding OTC derivative contracts equaled $\$ 24.7$ trillion by the end of 2012 with a whopping $\$ 632.6$ trillion in notional value (Bank for International Settlements (2013)). Adopting the risk-neutral valuation principle, we derive a general pricing equation for an OTC derivative deal where the new types of risks are included simply as modifications of the payout cash-flows. We address the current market practices in accordance with the guidelines of the International Swaps and Derivatives Association without assuming restrictive constraints on the collateral margining procedures and close-out netting rules. In particular, we allow for asymmetric collateral and funding rates as well as exogenously 
given liquidity policies and hedging strategies.

When dealing with funding costs, one may take a single deal (micro) or homogeneous (macro) cost view. In the micro view, funding costs are determined at deal level. This means that the trading desk may borrow funds at a different rate than at which it can invest funds. In a slightly more aggregate cost view, average funding spreads are applied to all deals yet the spread on borrowing funds may still be different from that on lending. Finally, if we turn to the macro view, funding costs of borrowing and lending are assumed the same and a common funding spread is applied across all deals. Clearly, the treasury department plays an active part in the micro approach and works as an operational center, while in the macro approach it takes more the role of a supporting function for the trading business. In this work we stay as general as possible and adopt a micro cost view. Naturally, the macro view is just a special case of the micro view.

The introduction of funding risk makes the pricing problem highly recursive. The price of the deal depends on the trader's funding strategy, while to determine the optimal funding strategy we need to know the deal price itself. This recursive structure was also discovered in the studies of Crépey (2011) and Burgard and Kjaer (2011b), yet the feature is neglected in the common approach of adding a funding spread to the discount curve. Valuation under funding risk poses a significantly more complex and computationally demanding problem than standard CVA and DVA computations since it requires forward simulation and backward induction at the same time. In addition, FVA does not take the form of a simple additive term as appears to be commonly assumed by market participants. More fundamentally, this means that, by its very nature, identifying FVA with DVA is generally wrong, and only under restrictive assumptions would the two concepts collapse. We show how the general pricing equation can be cast as a set of iterative equations that can be conveniently solved by means of least-squares Monte Carlo (see, e.g., Carriere (1996), Longstaff and Schwartz (2001), Tilley (1993), and Tsitsiklis and Van Roy (2001)) and we propose an efficient simulation algorithm. Also, we derive a continuous-time approximation of the solution of the pricing equation as well as the associated partial differential equation (PDE).

Despite its general market acceptance, the practice including an adjustment for funding costs has stirred quite some controversy among academics and practitioners (see the debate following Hull and White (2012)). At the center of this controversy is the issue that funding-contingent pricing becomes subjective due to asymmetric information. The particular funding policy chosen by the client is not (fully) known to the dealer, and vice 
versa. As a result, the price of the deal may be different to either of the two parties. Theoretically, this should mean that the parties would never close the deal. However, in reality, the dealer may not be able to recoup his full funding costs from the client, yet traders say that funding risk was the key factor driving bid-ask spreads wider during the crisis.

Several studies have analyzed the various valuation adjustments separately, but few have tried to build a valuation approach that consistently takes collateralization, counterparty credit risk, and funding costs into account. Under unilateral default risk, ie. when only one party is defaultable, Brigo and Masetti (2005) consider valuation of derivatives with CVA, while particular applications of their approach are given in Brigo and Pallavicini (2007), Brigo and Bakkar (2009), Brigo and Chourdakis (2009), and Brigo et al. (2011). Bilateral default risk appears in Bielecki and Rutkowski (2002), Brigo and Capponi (2008), Brigo et al. (2011), and Gregory (2009) who price both the CVA and DVA of a derivatives deal. The impact of collateralization on default risk has been investigated in Cherubini (2005) and more recently in Brigo et al. (2011) and Brigo et al. (2011).

Assuming no default risk, Piterbarg (2010) provides an initial analysis of collateralization and funding risk in a stylized Black-Scholes economy. Yet, the introduction of collateral in a world without default risk is questionable since its main purpose is to mitigate such risk. Morini and Prampolini (2011), Fries (2010) and Castagna (2011) consider basic implications of funding in presence of default risk. However, these works focus only on simple financial products, such as zero-coupon bonds or loans, and do not offer the level of generality needed to include all the required features. Thus, a general framework for consistent valuation under the new risks is still missing. The most comprehensive attempts are those of Burgard and Kjaer (2011b,a), Crépey (2011, 2012a,b), and Pallavicini et al. (2011, 2012). Nonetheless, as Burgard and Kjaer (2011b,a) resort to a PDE approach, their results are unrealistically constrained to low dimensions. Also, they neglect the hidden complexities of collateralization and mark-to-market discontinuities at default. The approach of Crépey $(2011,2012 a, b)$ is more general although it does not allow for credit instruments in the deal portfolio. We extend the work of Pallavicini et al. (2011, 2012) and develop a general pricing framework for OTC deals that fully and consistently takes collateralization, counterparty credit risk, and funding risk into account. The framework is conceptually simple and intuitive in contrast to previous attempts. It is based on the celebrated risk-neutral valuation principle and the new risks are included simply by 
adjusting the payout cash-flows of the deal. Finally, by resorting to Monte Carlo methods, the valuation framework can readily be used for high-dimensional problems.

The paper is organized as follows. Section 4.2 describes the general pricing framework with collateralized credit, debit, and funding valuation adjustments. Section 4.3 derives an iterative solution of the pricing equation as well as a continuous-time approximation. Section 4.4 describes a least-square Monte Carlo algorithm and provides numerical results on deal positions in European call options. Section 4.5 extends the pricing framework to cases where the dealer hedges the trade using other derivatives. Finally, Section 4.6 concludes the paper.

\subsection{Collateralized Credit and Funding Valuation Adjustments}

In this section we develop a general risk-neutral valuation framework for OTC derivative deals. The section clarifies how the traditional pre-crisis derivative price is consistently adjusted to reflect the new market realities of collateralization, counterparty credit risk, and funding risk. We refer to the two parties of a credit-risky deal as the investor or dealer ("I") on one side and the counterparty or client ("C") on the other.

Fixing the time horizon $T \in \mathbb{R}_{+}$of the deal, we define our risk-neutral pricing model on the probability space $\left(\Omega, \mathcal{G},\left(\mathcal{G}_{t}\right)_{t \in[0, T]}, \mathbb{Q}\right)$. $\mathbb{Q}$ is the risk-neutral probability measure. The filtration $\left(\mathcal{G}_{t}\right)_{t \in[0, T]}$ models the flow of information of the whole market, including credit, such that the default times of the investor $\tau_{I}$ and the counterparty $\tau_{C}$ are $\mathcal{G}$-stopping times. We adopt the notational convention that $\mathbb{E}_{t}$ is the risk-neutral expectation conditional on the information $\mathcal{G}_{t}$ while $\mathbb{E}_{\tau_{i}}$ denotes the conditional risk-neutral expectation given the stopped filtration $\mathcal{G}_{\tau_{i}}$. Moreover, we exclude the possibility of simultaneous defaults for simplicity and define the time of the first default event among the two parties as the stopping time

$$
\tau \triangleq\left(\tau_{I} \wedge \tau_{C}\right)
$$

In the sequel we adopt the view of the investor and consider the cash-flows and consequences of the deal from his perspective. In other words, when we price the deal we obtain the value of the position to the investor. As we will see, with funding risk this price will often not just be the value of the deal to the counterparty with opposite sign. 
The gist of the valuation framework is conceptually simple and rests neatly on the classical finance disciplines of risk-neutral pricing and discounting cash-flows. When a dealer enters into a derivatives deal with a client, a number of cash-flows are exchanged, and just like valuation of any other financial claim, discounting these cash in- or outflows gives us a price of the deal. Post-crisis market practice distinguishes four different types of cash-flow streams occurring once a trading position has been entered: (i) Cash-flows coming directly from the derivatives contract such as payoffs, coupons, dividends, etc. We denote by $\pi(t, T)$ the sum of the discounted payoff happening over the time period $(t, T]$. This is where classical derivatives pricing would usually stop and the price of a derivative contract with maturity $T$ would be given by

$$
V_{t}=\mathbb{E}_{t}[\pi(t, T)]
$$

This price assumes no credit risk of the parties involved and no funding risk of the trade. However, present-day market practice requires the price to be adjusted by taking further cash-flow transactions into account: (ii) Cash-flows required by collateral margining. If the deal is collateralized, cash flows happen in order to maintain a collateral account that in the case of default will be used to cover any losses. $\gamma(t, T ; C)$ is the sum of the discounted margining costs over the period $(t, T]$ with $C$ denoting the collateral account. (iii) Cash-flows exchanged once a default event has occurred. We let $\theta_{\tau}(C, \varepsilon)$ denote the on-default cash-flow with $\varepsilon$ being the residual value of the claim traded at default. Lastly, (iv) cash-flows required for funding the deal. We denote the sum of the discounted funding costs over the period $(t, T]$ by $\varphi(t, T ; F)$ with $F$ being the cash account needed for funding the deal. Collecting the terms we obtain a consistent price $\bar{V}$ of a derivative deal taking into account counterparty credit risk, margining costs, and funding costs

$$
\begin{aligned}
\bar{V}_{t}(C, F)=\mathbb{E}_{t}[ & \pi(t, T \wedge \tau)+\gamma(t, T \wedge \tau ; C)+\varphi(t, T \wedge \tau ; F) \\
+ & \left.\mathbf{1}_{\{t<\tau<T\}} D(t, \tau) \theta_{\tau}(C, \varepsilon)\right]
\end{aligned}
$$

where $D(t, \tau)$ is the risk-free discount factor.

By using a risk-neutral valuation approach, we see that only the payout needs to be adjusted under counterparty credit and funding risk. In the following paragraphs we expand the terms of (4.1) and carefully discuss how to compute them. 


\subsubsection{Trading under Collateralization and Close-out Netting}

The ISDA master agreement is the most commonly used framework for full and flexible documentation of OTC derivative transactions and is published by the International Swaps and Derivatives Association (ISDA (2009)). Once agreed between two parties, the master agreement sets out standard terms that apply to all deals entered into between those parties. The ISDA master agreement lists two tools to mitigate counterparty credit risk: collateralization and close-out netting. Collateralization of a deal means that the party which is out-of-the-money is required to post collateral - usually cash, government securities or highly rated bonds - corresponding to the amount payable by that party in the case of a default event. The credit support annex (CSA) to the ISDA master agreement defines the rules under which the collateral is posted or transferred between counterparties. Close-out netting means that in the case of default all transactions with the counterparty under the ISDA master agreement are consolidated into a single net obligation which then forms the basis for any recovery settlements.

Collateralization Collateralization of a deal usually happens according to a margining procedure. Such a procedure involves that both parties post collateral amounts to or withdraw amounts from the collateral account $C$ according to their current exposure on pre-fixed dates $\left\{t_{1}, \ldots, t_{n}=T\right\}$ during the life of the deal, typically daily. The terms of the margining procedure may, furthermore, include independent amounts, minimum transfer amounts, thresholds, etc., as described in Brigo et al. (2011). However, here we adopt a general description of the margining procedure that does not rely on the particular terms chosen by the parties.

We consider a collateral account $C$ held by the investor. Moreover, we assume that the investor is the collateral taker when $C_{t}>0$ and the collateral provider when $C_{t}<0$. The CSA ensures that the collateral taker remunerates the account $C$ at an accrual rate. If the investor is collateral taker, he remunerates the collateral account by the accrual rate $c_{t}^{+}(T)$, while if he is the collateral provider, the counterparty remunerates the account at the rate $c_{t}^{-}(T)^{1}$. The effective accrual collateral rate $\tilde{c}_{t}(T)$ is defined as

$$
\tilde{c}_{t}(T) \triangleq c_{t}^{-}(T) \mathbf{1}_{\left\{C_{t}<0\right\}}+c_{t}^{+}(T) \mathbf{1}_{\left\{C_{t}>0\right\}},
$$

\footnotetext{
${ }^{1}$ We stress the slight abuse of notation here: A plus and minus sign does not indicate that the rates are positive or negative parts of some other rate, but instead it tells which rate is used to accrue interest on the collateral according to the sign of the collateral account.
} 
To understand the cash-flows originating from collateralization of the deal, let us consider the consequences of the margining procedure to the investor. At the first margin date, say $t_{1}$, the investor opens the account and posts collateral if he is out-of-the-money, i.e. if $C_{t_{1}}<0$, which means that the counterparty is the collateral taker. On each of the following margin dates $t_{k}$, the investor posts collateral according to his exposure as long as $C_{t_{k}}<0$. As collateral taker, the counterparty pays interest on the collateral at the accrual rate $c_{t_{k}}^{-}\left(t_{k+1}\right)$ between the following margin dates $t_{k}$ and $t_{k+1}$. We assume that interest accrued on the collateral is saved into the account and thereby directly included in the margining procedure and the close-out. Finally, if $C_{t_{n}}<0$ on the last margin date $t_{n}$, the investor closes the collateral account given no default event has occurred in between. Similarly, for positive values of the collateral account, the investor is instead the collateral taker and the counterparty faces corresponding cash-flows at each margin date. If we sum up all the discounted margining cash-flows of the investor and the counterparty, we obtain

$$
\begin{aligned}
\Gamma(t, T \wedge \tau ; C)= & \sum_{k=1}^{n-1} \mathbf{1}_{\left\{t_{k}<\tau\right\}}\left(D\left(t, t_{k}\right) C_{t_{k}}-D\left(t, t_{k+1}\right) \mu\left(t_{k}, t_{k+1}\right)\right) \\
& +\sum_{k=1}^{n-1} \mathbf{1}_{\left\{t_{k}<\tau<t_{k+1}\right\}} D\left(t, t_{k+1}\right) \mu\left(t_{k}, t_{k+1}\right),
\end{aligned}
$$

where

$$
\mu\left(t_{k}, t_{k+1}\right) \triangleq \frac{C_{t_{k}}^{-}}{P_{t_{k}}^{c^{-}}\left(t_{k+1}\right)}+\frac{C_{t_{k}}^{+}}{P_{t_{k}}^{c^{+}}\left(t_{k+1}\right)},
$$

denotes the value of the collateral account accrued from date $t_{k}$ to date $t_{k+1}$ as required by the CSA, and the (collateral) zero-coupon bond is defined as $P_{t}^{c^{ \pm}}(T) \triangleq[1+(T-$ t) $\left.c^{ \pm}{ }_{t}(T)\right]^{-1}$. We use the short-hand notation $\mathcal{X}^{+} \triangleq \max (\mathcal{X}, 0)$ and $\mathcal{X}^{-} \triangleq \min (\mathcal{X}, 0)$ for the positive and negative parts of a random variable $\mathcal{X}$.

From (4.3) the price of the collateral margining cash-flows is obtained by taking the risk-neutral expectation

$$
\mathbb{E}_{t}[\Gamma(t, T \wedge \tau ; C)]=\mathbb{E}_{t}\left[\gamma(t, T \wedge \tau ; C)+\mathbf{1}_{\{\tau<T\}} D(t, \tau) C_{\tau^{-}}\right]
$$

where the collateral margining costs $\gamma(t, T \wedge \tau ; C)$ entering (4.1) are defined as

$$
\gamma(t, T \wedge \tau ; C) \triangleq \sum_{k=1}^{n-1} \mathbf{1}_{\left\{t \leqslant t_{k}<(T \wedge \tau)\right\}} D\left(t, t_{k}\right) C_{t_{k}}\left(1-\frac{P_{t_{k}}\left(t_{k+1}\right)}{P_{t_{k}}^{\tilde{c}}\left(t_{k+1}\right)}\right),
$$


with the zero-coupon bond $P_{t}^{\tilde{c}}(T) \triangleq\left[1+(T-t) \tilde{c}_{t}(T)\right]^{-1}$, and the pre-default value $C_{\tau^{-}}$ of the collateral account is given by

$$
C_{\tau^{-}} \triangleq \sum_{k=1}^{n-1} \mathbf{1}_{\left\{t_{k}<\tau<t_{k+1}\right\}} C_{t_{k}} \frac{P_{\tau}\left(t_{k+1}\right)}{P_{t_{k}}^{\tilde{c}}\left(t_{k+1}\right)} .
$$

In accordance with the CSA, this pre-default value of the collateral account is used to compute the netted exposure at close-out. It may be different from the actual value of the collateral account at default since part or all of the collateral may be rehypothecated. We discuss rehypothecation and its inherent liquidity risk in the following.

Rehypothecation Often the CSA grants the collateral taker relatively unrestricted use of the collateral for his liquidity and trading needs until it is returned to the collateral provider. Effectively, the practice of rehypocthecation lowers the costs of remuneration of the provided collateral. However, while without rehypothecation the collateral provider can expect to get any excess collateral returned after honoring the amount payable on the deal, if rehypothecation is allowed the collateral provider runs the risk of losing a fraction or all of the excess collateral in case of default on the collateral taker's part.

We denote the recovery fraction on the rehypothecated collateral by $R_{I}^{\prime}$ when the investor is the collateral taker and by $R_{C}^{\prime}$ when the counterparty is the collateral taker. The general recovery fraction on the market value of the deal that the investor receives in the case of default of the counterparty is denoted by $R_{C}$, while $R_{I}$ is the recovery fraction received by the counterparty if the investor defaults. The collateral provider typically has precedence over other creditors of the defaulting party in getting back any excess capital, which means $R_{I} \leqslant R_{I}^{\prime} \leqslant 1$ and $R_{C} \leqslant R_{C}^{\prime} \leqslant 1$. If no rehypothecation is allowed and the collateral is kept safe in a segregated account, we have that $R_{I}^{\prime}=R_{C}^{\prime}=1$.

Close-out netting In case of default all terminated transactions under the ISDA master agreement with a given counterparty are netted and consolidated into a single claim. This also includes any posted collateral to back the transactions. In this context the close-out amount plays a central role in calculating the on-default cash-flows. The close-out amount is the costs or losses that the surviving party incurs when replacing the terminated deal with an economic equivalent. Clearly, the size of these costs will depend on which party survives so we define the close-out amount as

$$
\varepsilon_{\tau} \triangleq \mathbf{1}_{\left\{\tau=\tau_{C}<\tau_{I}\right\}} \varepsilon_{I, \tau}+\mathbf{1}_{\left\{\tau=\tau_{I}<\tau_{C}\right\}} \varepsilon_{C, \tau}
$$


where $\varepsilon_{I, \tau}$ is the close-out amount on the counterparty's default priced at time $\tau$ by the investor and $\varepsilon_{C, \tau}$ is the close-out amount if the investor defaults. Recall that we always consider the deal from the investor's viewpoint in terms of the sign of the cash-flows involved. This means that if the close-out amount $\varepsilon_{I, \tau}$ as measured by the investor is positive, the investor is a creditor of the counterpaty, while if it is negative, the investor is a debtor of the counterparty. Analogously, if the close-out amount $\varepsilon_{C, \tau}$ to the counterparty but viewed from the investor is positive, the investor is a creditor of the counterparty, and if it is negative, the investor is a debtor to the counterparty.

We note that the ISDA documentation is, in fact, not very specific in terms of how to actually calculate the close-out amount. Since 2009 ISDA has allowed for the possibility to switch from a risk-free close-out rule to a replacement rule that includes the DVA of the surviving party in the recoverable amount. Parker and McGarry (2009) and Weeber and Robson (2009) show how a wide range of values of the close-out amount can be produced within the terms of ISDA. We refer to Brigo et al. (2011) and the references therein for further discussions on these issues. Here, we adopt the approach of Brigo et al. (2011) listing the cash-flows of all the various scenarios that can occur if default happens. We will net the exposure against the pre-default value of the collateral $C_{\tau-}$ and treat any remaining collateral as an unsecured claim. If we aggregate all these cash-flows and the pre-default value of collateral account, we reach the following expression for the on-default cash-flow

$$
\begin{aligned}
\theta_{\tau}(C, \varepsilon) \triangleq & \mathbf{1}_{\left\{\tau=\tau_{C}<\tau_{I}\right\}}\left(\varepsilon_{I, \tau}-\operatorname{LGD}_{C}\left(\varepsilon_{I, \tau}^{+}-C_{\tau^{-}}^{+}\right)^{+}-\operatorname{LGD}_{C}^{\prime}\left(\varepsilon_{I, \tau}^{-}-C_{\tau^{-}}^{-}\right)^{+}\right) \\
& +\mathbf{1}_{\left\{\tau=\tau_{I}<\tau_{C}\right\}}\left(\varepsilon_{C, \tau}-\operatorname{LGD}_{I}\left(\varepsilon_{C, \tau}^{-}-C_{\tau^{-}}^{-}\right)^{-}-\operatorname{LGD}_{I}^{\prime}\left(\varepsilon_{C, \tau}^{+}-C_{\tau^{-}}^{+}\right)^{-}\right) .
\end{aligned}
$$

where we define the loss-given-default as $\mathrm{LGD}_{C} \triangleq 1-R_{C}$, and the collateral loss-givendefault as $\mathrm{LGD}_{C}^{\prime} \triangleq 1-R_{C}^{\prime}$. If both parties agree on the exposure, namely $\varepsilon_{I, \tau}=\varepsilon_{C, \tau}=\varepsilon_{\tau}$, when we take the risk-neutral expectation in (4.1), we see that the price of the discounted on-default cash-flow,

$$
\left.\mathbb{E}_{t}\left[\mathbf{1}_{\{t<\tau<T\}} D(t, \tau) \theta_{\tau}(C, \varepsilon)\right]=\mathbb{E}_{t}\left[\mathbf{1}_{\{t<\tau<T\}} D(t, \tau) \varepsilon_{\tau}\right)\right]-\operatorname{Cva}(t, T ; C)-\operatorname{Dva}(t, T ; C),
$$

is the present value of the close-out amount reduced by the collateralized CVA and DVA terms

$$
\begin{aligned}
& \operatorname{CvA}(t, T ; C) \triangleq \mathbb{E}_{t}\left[\mathbf{1}_{\left\{\tau=\tau_{C}<T\right\}} D(t, \tau)\left(\operatorname{LGD}_{C}\left(\varepsilon_{I, \tau}^{+}-C_{\tau^{-}}^{+}\right)^{+}+\operatorname{LGD}_{C}^{\prime}\left(\varepsilon_{I, \tau}^{-}-C_{\tau^{-}}^{-}\right)^{+}\right)\right], \\
& \operatorname{DvA}(t, T ; C) \triangleq \mathbb{E}_{t}\left[\mathbf{1}_{\left\{\tau=\tau_{I}<T\right\}} D(t, \tau)\left(\operatorname{LGD}_{I}\left(\varepsilon_{C, \tau}^{-}-C_{\tau^{-}}^{-}\right)^{-}+\operatorname{LGD}_{I}^{\prime}\left(\varepsilon_{C, \tau}^{+}-C_{\tau^{-}}^{+}\right)^{-}\right)\right]
\end{aligned}
$$


Also, observe that if rehypothecation of the collateral is not allowed, the terms multiplied by $\mathrm{LGD}_{C}^{\prime}$ and $\mathrm{LGD}_{I}^{\prime}$ drops out of the CVA and DVA calculations.

\subsubsection{Trading under Funding Risk}

The hedging strategy that perfectly replicates the no-arbitrage price of a derivative is formed by a position in cash and a position in a portfolio of hedging instruments. When we talk about a derivative deal's funding, we essentially mean the cash position that is required as part of the hedging strategy, and with funding costs we refer to the costs of maintaining this cash position. If we denote the cash account by $F$ and the risky-asset account by $H$, we get

$$
\bar{V}_{t}=F_{t}+H_{t}
$$

In the classical Black-Scholes-Merton theory, the risky part $H$ of the hedge would be a delta position in the underlying stock, whereas the risk-less part $F$ would be a position in the risk-free bank account. If the deal is collateralized, the margining procedure is included in the deal definition insuring that funding of the collateral is automatically taken into account. Moreover, if rehypothecation is allowed for the collateralized deal, the collateral taker can use the posted collateral as a funding source and thereby reduce or maybe even eliminate the costs of funding the deal. Thus, we have the following two definitions of the funding account: If rehypothecation of the posted collateral is allowed,

$$
F_{t} \triangleq \bar{V}_{t}(C, F)-C_{t}-H_{t}
$$

and if such rehypothecation is forbidden, we have

$$
F_{t} \triangleq \bar{V}_{t}(C, F)-H_{t}
$$

By implication of (4.10) and (4.11) it is obvious that if the funding account $F_{t}>0$, the dealer needs to borrow cash to establish the hedging strategy at time $t$. Correspondingly, if the funding account $F_{t}<0$, the hedging strategy requires the dealer to invest surplus cash. Specifically, we assume the dealer enters a funding position on a discrete time-grid $\left\{t_{1}, \ldots, t_{m}\right\}$ during the life of the deal. Given two adjacent funding times $t_{j}$ and $t_{j+1}$, for $1 \leq j \leq m-1$, the dealer enters a position in cash equal to $F_{t_{j}}$ at time $t_{j}$. At time $t_{j+1}$ the dealer redeems the position again and either returns the cash to the funder if it was a long cash position and pays funding costs on the borrowed cash, or he gets the cash back if it was a short cash position and receives funding benefits as interest on the invested 
cash. We assume that these funding costs and benefits are determined at the start date of each funding period and charged at the end of the period.

Without any loss of generality, the contracts used by the investor to fund the deal can be introduced as adapted price processes. Let $P_{t}^{f^{+}}(T)$ represent the price of a borrowing contract measurable at $t$ where the dealer pays one unit of cash at maturity $T>t$, and let $P_{t}^{f^{-}}(T)$ be the price of a lending contract where the dealer receives one unit of cash at maturity. Moreover, the corresponding accrual rates are given by

$$
f_{t}^{ \pm}(T) \triangleq \frac{1}{T-t}\left(\frac{1}{P_{t}^{f^{ \pm}}(T)}-1\right)
$$

In other words, if the hedging strategy of the deal requires borrowing cash, this can be done at the funding frate $f^{+}$, while surplus cash can be invested at the lending rate $f^{-}$. We define the effective funding rate $\tilde{f}_{t}$ faced by the dealer as

$$
\tilde{f}_{t}(T) \triangleq f_{t}^{-}(T) \mathbf{1}_{\left\{F_{t}<0\right\}}+f_{t}^{+}(T) \mathbf{1}_{\left\{F_{t}>0\right\}} \cdot
$$

Following this, the sum of discounted cash-flows from funding the hedging strategy during the life of the deal is equal to

$$
\varphi(t, T \wedge \tau ; F) \triangleq \sum_{j=1}^{m-1} \mathbf{1}_{\left\{t \leqslant t_{j}<(T \wedge \tau)\right\}} D\left(t, t_{j}\right) F_{t_{j}}\left(1-\frac{P_{t_{j}}\left(t_{j+1}\right)}{P_{t_{j}}^{\tilde{f}}\left(t_{j+1}\right)}\right),
$$

where the zero-coupon bond corresponding to the effective funding rate is defined as $P_{t}^{\tilde{f}}(T) \triangleq\left[1+(T-t) \tilde{f}_{t}(T)\right]^{-1}$. This is, strictly speaking, a discounted payout and the funding cost or benefit at time $t$ is obtained by taking the risk neutral expectation of the above cash-flows.

The particular positions entered by the dealer to either borrow or invest cash according to the sign and size of the funding account depend on the bank's liquidity policy. In the following we discuss two possible cases: One where the dealer can fund at rates set by the bank's treasury department, and another where the dealer goes to the market directly and funds his trades at the prevailing market rates. As a result, the funding rates and therefore the funding effect on the price of a derivative deal depends intimately on the chosen liquidity policy.

Treasury Funding If the dealer funds the hedge through the bank's treasury department, the treasury determines the funding rates $f^{ \pm}$faced by the dealer, often assuming 
average funding costs and benefits across all deals. This leads to two curves as functions of maturity; one for borrowing funds $f^{+}$and one for lending funds $f^{-}$. After entering a funding position $F_{t_{j}}$ at time $t_{j}$, the dealer faces the following discounted cash-flow

$$
\Phi_{j}\left(t_{j}, t_{j+1} ; F\right) \triangleq-N_{t_{j}} D\left(t_{j}, t_{j+1}\right)
$$

with

$$
N_{t_{j}} \triangleq \frac{F_{t_{j}}^{-}}{P_{t_{j}}^{f^{-}}\left(t_{j+1}\right)}+\frac{F_{t_{j}}^{+}}{P_{t_{j}}^{f^{+}}\left(t_{j+1}\right)} .
$$

Under this liquidity policy, the treasury - and not the dealer himself - is in charged of debt valuation adjustments due to funding-related positions. Also, being entities of the same institution, both the dealer and the treasury disappear in case of default of the institution without any further cash-flows being exchanged and we can neglect the effects of funding in this case. So, when default risk is considered, this leads to following definition of the funding cash flows

$$
\bar{\Phi}_{j}\left(t_{j}, t_{j+1} ; F\right) \triangleq \mathbf{1}_{\left\{\tau>t_{j}\right\}} \Phi_{j}\left(t_{j}, t_{j+1} ; F\right) .
$$

Thus, the risk-neutral price of the cash-flows due to the funding positions entered at time $t_{j}$ is

$$
\mathbb{E}_{t_{j}}\left[\bar{\Phi}_{j}\left(t_{j}, t_{j+1} ; F\right)\right]=-\mathbf{1}_{\left\{\tau>t_{j}\right\}}\left(F_{t_{j}}^{-} \frac{P_{t_{j}}\left(t_{j+1}\right)}{P_{t_{j}}^{f^{-}}\left(t_{j+1}\right)}+F_{t_{j}}^{+} \frac{P_{t_{j}}\left(t_{j+1}\right)}{P_{t_{j}}^{f^{+}}\left(t_{j+1}\right)}\right) .
$$

If we consider a sequence of such funding operations at each time $t_{j}$ during the life of the deal, we can define the sum of cash-flows coming from all the borrowing and lending positions opened by the dealer to hedge the trade up to the first-default event

$$
\begin{aligned}
\varphi(t, T \wedge \tau ; F) & \triangleq \sum_{j=1}^{m-1} \mathbf{1}_{\left\{t \leqslant t_{j}<(T \wedge \tau)\right\}} D\left(t, t_{j}\right)\left(F_{t_{j}}+\mathbb{E}_{t_{j}}\left[\bar{\Phi}_{j}\left(t_{j}, t_{j+1} ; F\right)\right]\right) \\
& =\sum_{j=1}^{m-1} \mathbf{1}_{\left\{t \leqslant t_{j}<(T \wedge \tau)\right\}} D\left(t, t_{j}\right)\left(F_{t_{j}}-F_{t_{j}}^{-} \frac{P_{t_{j}}\left(t_{j+1}\right)}{P_{t_{j}}^{f^{-}}\left(t_{j+1}\right)}-F_{t_{j}}^{+} \frac{P_{t_{j}}\left(t_{j+1}\right)}{P_{t_{j}}^{f^{+}}\left(t_{j+1}\right)}\right)
\end{aligned}
$$

In terms of the effective funding rate, this expression collapses to (4.13).

Market Funding If the dealer funds the hedging strategy in the market - and not through the bank's treasury - the funding rates are determined by prevailing market conditions and are often deal specific. This means that the rate $f^{+}$the dealer can borrow funds at may be different from the rate $f^{-}$at which funds can be invested. Moreover, 
these rates may differ across deals depending on the deals' notional, maturity structures, dealer-client relationship, and so forth. Similar to the liquidity policy of treasury funding, we assume a deal's funding operations are closed down in the case of default. Furthermore, as the dealer now operates directly on the market, he needs to include a DVA due to his funding positions when he marks-to-market his trading books. For simplicity, we assume that the funder in the market is default-free so no funding CVA needs to be accounted for. The discounted cash-flow from the borrowing or lending position between two adjacent funding times $t_{j}$ and $t_{j+1}$ is given by

$$
\begin{aligned}
\bar{\Phi}_{j}\left(t_{j}, t_{j+1} ; F\right) \triangleq & \mathbf{1}_{\left\{\tau>t_{j}\right\}} \mathbf{1}_{\left\{\tau_{I}>t_{j+1}\right\}} \Phi_{j}\left(t_{j}, t_{j+1} ; F\right) \\
& -\mathbf{1}_{\left\{\tau>t_{j}\right\}} \mathbf{1}_{\left\{\tau_{I}<t_{j+1}\right\}}\left(\mathrm{LGD}_{I} \varepsilon_{F, \tau_{I}}^{-}-\varepsilon_{F, \tau_{I}}\right) D\left(t_{j}, \tau_{I}\right),
\end{aligned}
$$

where $\varepsilon_{F, t}$ is the close-out amount calculated by the funder on the dealer's default

$$
\varepsilon_{F, \tau_{I}} \triangleq-N_{t_{j}} P_{\tau_{I}}\left(t_{j+1}\right)
$$

To price this funding cash-flow, we take the risk-neutral expectation

$$
\mathbb{E}_{t_{j}}\left[\bar{\Phi}_{j}\left(t_{j}, t_{j+1} ; F\right)\right]=-\mathbf{1}_{\left\{\tau>t_{j}\right\}}\left(F_{t_{j}}^{-} \frac{P_{t_{j}}\left(t_{j+1}\right)}{P_{t_{j}}^{f^{-}}\left(t_{j+1}\right)}+F_{t_{j}}^{+} \frac{P_{t_{j}}\left(t_{j+1}\right)}{\bar{P}_{t_{j}}^{f^{+}}\left(t_{j+1}\right)}\right)
$$

Here, the zero-coupon funding bond $\bar{P}_{t}^{f^{+}}(T)$ for borrowing cash is adjusted for the dealer's credit risk

$$
\bar{P}_{t}^{f^{+}}(T) \triangleq \frac{P_{t}^{f^{+}}(T)}{\mathbb{E}_{t}^{T}\left[\operatorname{LGD}_{I} \mathbf{1}_{\left\{\tau_{I}>T\right\}}+R_{I}\right]},
$$

where the expectation on the right-hand side is taken under the $T$-forward measure. Naturally, since the seniority could be different, one might assume a different recovery rate on the funding position than on the derivatives deal itself (see Crépey (2011)). Extensions to this case are straightforward.

Next, summing the discounted cash-flows from the sequence of funding operations through the life the deal, we get

$$
\begin{aligned}
\varphi(t, T \wedge \tau ; F) & \triangleq \sum_{j=1}^{m-1} \mathbf{1}_{\left\{t \leqslant t_{j}<T \wedge \tau\right\}} D\left(t, t_{j}\right)\left(F_{t_{j}}+\mathbb{E}_{t_{j}}\left[\bar{\Phi}_{j}\left(t_{j}, t_{j+1} ; F\right)\right]\right) \\
& =\sum_{j=1}^{m-1} \mathbf{1}_{\left\{t \leqslant t_{j}<T \wedge \tau\right\}} D\left(t, t_{j}\right)\left(F_{t_{j}}-F_{t_{j}}^{-} \frac{P_{t_{j}}\left(t_{j+1}\right)}{P_{t_{j}}^{f^{-}}\left(t_{j+1}\right)}-F_{t_{j}}^{+} \frac{P_{t_{j}}\left(t_{j+1}\right)}{\bar{P}_{t_{j}}^{f^{+}}\left(t_{j+1}\right)}\right) .
\end{aligned}
$$


To avoid cumbersome notation, we will not explicitly write $\bar{P}^{f^{+}}$in the sequel, but just keep in mind that when the dealer funds directly in the market then $P^{f^{+}}$needs to be adjusted for funding DVA. Thus, in terms of the effective funding rate, we obtain (4.13).

\subsection{General Pricing Equation for OTC Trades}

In the previous section we analyzed the discounted cash-flows of a derivative deal and we developed a framework for consistent valuation of such deals under collateralized counterparty credit and funding risk. The arbitrage-free pricing framework is captured in the following theorem.

Theorem 6 (General Pricing Equation). The consistent arbitrage-free price $\bar{V}_{t}(C, F)$ of collateralized OTC derivative deals with counterparty credit risk and funding costs takes the form

$$
\begin{gathered}
\bar{V}_{t}(C, F)=\mathbb{E}_{t}[\pi(t, T \wedge \tau)+\gamma(t, T \wedge \tau ; C)+\varphi(t, T \wedge \tau ; F) \\
\left.+\mathbf{1}_{\{t<\tau<T\}} D(t, \tau) \theta_{\tau}(C, \varepsilon)\right]
\end{gathered}
$$

where

1. $\pi(t, T \wedge \tau)$ is the discounted cash-flows from the contract's payoff structure up to the first-default event.

2. $\gamma(t, T \wedge \tau ; C)$ is the discounted cash-flows from the collateral margining procedure up to the first-default event and is defined in (4.4).

3. $\varphi(t, T \wedge \tau ; F)$ is the discounted cash-flows from funding the hedging strategy up to the first-default event and is defined in (4.13).

4. $\theta_{\tau}(C, \varepsilon)$ is the on-default cash-flow with close-out amount $\varepsilon$ and is defined in (4.7).

If funding and collateral margining costs are discarded, this pricing equation collapses to the formula derived in Brigo et al. (2011) for the price of a derivative under bilateral counterparty credit risk.

While the pricing equation is conceptually clear - we simply take the expectation of the sum of all discounted cash-flows of the deal under the risk-neutral measure - solving the 
equation poses a highly recursive, non-linear problem. The future paths of the effective funding rate $\tilde{f}$ depend on the future signs of the funding account $F$, i.e. whether we need to borrow or lend cash on each future funding date. At the same time, through the relations (4.10) and (4.11), the future sign and size of the funding account $F$ depend on the adjusted price $\bar{V}$ of the deal which is the quantity we are trying to compute in the first place. One crucial implication of this recursive structure of the pricing problem is the fact that FVA is generally not just an additive adjustment term, in contrast to CVA and DVA. More importantly, the conjecture identifying the DVA of a deal with its funding is wrong in general. Only in the unrealistic setting where the dealer can fund an uncollateralized trade at equal borrowing and lending rates, i.e. $f^{+}=f^{-}$, do we achieve the additive structure often assumed by practitioners. If the trade is collateralized, we need to impose even further restrictions as to how the collateral is linked to price of the trade $\bar{V}$.

On the theoretical side, the pricing equation shakes the foundation of the celebrated Law of One Price prevailing in classical derivatives pricing. Clearly, if we assume no funding costs, the dealer and counterparty agree on the price of the deal as both parties can - at least theoretically - observe the credit risk of each other through CDS contracts traded in the market and thus agree on the CVA and DVA. In contrast, introducing funding costs, they will not agree on the FVA for the deal due to asymmetric information. The parties cannot observe each others' liquidity policies nor their respective funding costs associated with a particular deal. As a result, the value of a deal position will not generally be the same to the counterparty as to the dealer just with opposite sign. In principle, this should mean that the dealer and the counterparty would never close the trade, but in practice trades are executed as a simple consequence of the fundamental forces of supply and demand. Nevertheless, among dealers it is the general belief that funding costs were one of the main factors driving the bid-ask spreads wider during the recent financial crisis.

Finally, as we adopt a risk-neutral valuation framework, we implicitly assume the existence of a risk-free interest rate. Indeed, since the valuation adjustments are included as additional cash-flows and not as ad-hoc spreads, all the cash-flows are discounted by the risk-free discount factor $D(t, T)$ in (4.14). Nevertheless, the risk-free rate is merely an instrumental variable of the general pricing equation. We clearly distinguish market rates from the theoretical risk-free rate avoiding the false claim that the over-night rates (e.g., EONIA) are risk free. In fact, as we will show in continuous time, if the dealer funds the hedging strategy of the trade through cash accounts available to him - whether 
as rehypothecated collateral or funds from the treasury, repo market, etc. - the risk-free rate vanishes from the pricing equation.

\subsubsection{Discrete-time Solution}

Our purpose here is to turn the recursive pricing equation (4.14) into a set of iterative equations that can be solved by least-squares Monte Carlo methods. These methods are already standard in CVA and DVA calculations (Brigo and Pallavicini (2007)). To this end, we introduce the auxiliary function

$$
\bar{\pi}\left(t_{j}, t_{j+1} ; C\right) \triangleq \pi\left(t_{j}, t_{j+1} \wedge \tau\right)+\gamma\left(t_{j}, t_{j+1} \wedge \tau ; C\right)+\mathbf{1}_{\left\{t_{j}<\tau<t_{j+1}\right\}} D\left(t_{j}, \tau\right) \theta_{\tau}(C, \varepsilon)
$$

which defines the cash-flows of the deal occurring between time $t_{j}$ and $t_{j+1}$ adjusted for collateral margining costs and default risks. We stress the fact that the close-out amount used for calculating the on-default cash-flow still refers to a deal with maturity $T$. If we then solve pricing equation (4.14) at each funding date $t_{j}$ in the time-grid $\left\{t_{1}, \ldots, t_{n}=T\right\}$, we obtain the deal price $\bar{V}$ at time $t_{j}$ as a function of the deal price on the next consecutive funding date $t_{j+1}$

$$
\begin{aligned}
\bar{V}_{t_{j}}(C ; F)= & \mathbb{E}_{t_{j}}\left[\bar{V}_{t_{j+1}}(C ; F) D\left(t_{j}, t_{j+1}\right)+\bar{\pi}\left(t_{j}, t_{j+1} ; C\right)\right] \\
& +\mathbf{1}_{\left\{\tau>t_{j}\right\}}\left(F_{t_{j}}-F_{t_{j}}^{-} \frac{P_{t_{j}}\left(t_{j+1}\right)}{P_{t_{j}}^{f^{-}}\left(t_{j+1}\right)}-F_{t_{j}}^{+} \frac{P_{t_{j}}\left(t_{j+1}\right)}{P_{t_{j}}^{f^{+}}\left(t_{j+1}\right)}\right),
\end{aligned}
$$

where, by definition, $\bar{V}_{t_{n}}(C ; F) \triangleq 0$ on the final date $t_{n}$. Recall the definitions of the funding account in (4.10) if no rehypothecation of collateral is allowed and in (4.11) if rehypothecation is permitted, we can then solve the above equation for the positive and negative parts of the funding account. The outcome of this exercise is a discrete-time iterative solution of the recursive pricing equation, provided in the following theorem.

Theorem 7 (Discrete-time Solution of the General Pricing Equation). We may solve the full recursive pricing equation in Theorem 6 as a set of backward-iterative equations on the time-grid $\left\{t_{1}, \ldots, t_{n}=T\right\}$ with $\bar{V}_{t_{n}}(C ; F) \triangleq 0$. For $\tau<t_{j}$, we have

$$
\bar{V}_{t_{j}}(C ; F)=0
$$

while for $\tau>t_{j}$, we have 
(i) if re-hypothecation is forbidden:

$$
\left(\bar{V}_{t_{j}}(C ; F)-H_{t_{j}}\right)^{ \pm}=P_{t_{j}}^{\tilde{f}}\left(t_{j+1}\right)\left(\mathbb{E}_{t_{j}}^{t_{j+1}}\left[\bar{V}_{t_{j+1}}(C ; F)+\frac{\bar{\pi}\left(t_{j}, t_{j+1} ; C\right)-H_{t_{j}}}{D\left(t_{j}, t_{j+1}\right)}\right]\right)^{ \pm}
$$

(ii) if re-hypothecation is allowed:

$$
\begin{aligned}
\left(\bar{V}_{t_{j}}(C ; F)-C_{t_{j}}-H_{t_{j}}\right)^{ \pm} & = \\
& P_{t_{j}}^{\tilde{f}}\left(t_{j+1}\right)\left(\mathbb{E}_{t_{j}}^{t_{j+1}}\left[\bar{V}_{t_{j+1}}(C ; F)+\frac{\bar{\pi}\left(t_{j}, t_{j+1} ; C\right)-C_{t_{j}}-H_{t_{j}}}{D\left(t_{j}, t_{j+1}\right)}\right]\right)^{ \pm},
\end{aligned}
$$

where the expectations are taken under the $\mathbb{Q}^{t_{j+1}}$-forward measure.

The \pm sign in the theorem is supposed to stress the fact that the sign of the funding account, which determines the effective funding rate, depends on the sign of the conditional expectation. Further intuition may be gained by going to continuous time which is the case we will now turn to.

\subsubsection{Continuous-time Solution}

Let us consider a continuous-time approximation of the general pricing equation. This implies that collateral margining, funding, and hedging strategies are executed in continuous time. Moreover, we assume that rehypothecation is allowed, but similar results hold if this is not the case. By taking the time limit, we have the following expressions for the discounted cash-flow streams of the deal

$$
\begin{gathered}
\pi(t, T \wedge \tau)=\int_{t}^{T \wedge \tau} \Pi(s, s+d s) D(t, s), \quad \gamma(t, T \wedge \tau ; C)=\int_{t}^{T \wedge \tau}\left(r_{s}-\tilde{c}_{s}\right) C_{s} D(t, s) d s \\
\varphi(t, T \wedge \tau ; F)=\int_{t}^{T \wedge \tau}\left(r_{s}-\tilde{f}_{s}\right) F_{s} D(t, s) d s
\end{gathered}
$$

where $\Pi(t, t+d t)$ is the payoff coupon process of the derivative contract and $r_{t}$ is the risk-free rate. Then, putting all the above terms together with the on-default cash flow as in Theorem 6 , the recursive pricing equation yields

$$
\begin{aligned}
\bar{V}_{t}(C ; F)= & \int_{t}^{T} \mathbb{E}_{t}\left[\left(\mathbf{1}_{\{s<\tau\}} \Pi(s, s+d s)+\mathbf{1}_{\{\tau \in d s\}} \theta_{s}(C, \varepsilon)\right) D(t, s)\right] \\
& +\int_{t}^{T} \mathbb{E}_{t}\left[\mathbf{1}_{\{s<\tau\}}\left(\tilde{f}_{s}-\tilde{c}_{s}\right) C_{s} D(t, s)\right] d s \\
& +\int_{t}^{T} \mathbb{E}_{t}\left[\mathbf{1}_{\{s<\tau\}}\left(r_{s}-\tilde{f}_{s}\right)\left(\bar{V}_{s}(C, F)-H_{s}\right) D(t, s)\right] d s .
\end{aligned}
$$


We now switch to the market filtration $\mathcal{F}$. This step implicitly assumes a separable structure of our filtration $\mathcal{G}$. In other words, $\mathcal{G}$ is generated by the pure default-free market filtration $\mathcal{F}$ and the filtration generated by all the relevant default times (see for example Bielecki and Rutkowski (2002)). This allows us to rewrite the previous price equation as

$$
\begin{aligned}
\bar{V}_{t}(C ; F)= & \mathbf{1}_{\{\tau>t\}} \int_{t}^{T} \mathbb{E}_{t}\left[\left(\Pi(s, s+d s)+\lambda_{s} \theta_{s}(C, \varepsilon) d s\right) D(t, s ; r+\lambda) \mid \mathcal{F}\right] \\
& +\mathbf{1}_{\{\tau>t\}} \int_{t}^{T} \mathbb{E}_{t}\left[\left(\tilde{f}_{s}-\tilde{c}_{s}\right) C_{s} D(t, s ; r+\lambda) \mid \mathcal{F}\right] d s \\
& +\mathbf{1}_{\{\tau>t\}} \int_{t}^{T} \mathbb{E}_{t}\left[\left(r_{s}-\tilde{f}_{s}\right)\left(\bar{V}_{s}(C, F)-H_{s}\right) D(t, s ; r+\lambda) \mid \mathcal{F}\right] d s
\end{aligned}
$$

where $\lambda_{t}$ is the first-to-default intensity and the discount factor is defined as $D(t, s ; \xi) \triangleq$ $e^{-\int_{t}^{s} \xi_{u} d u}$. Assuming the conditions are satisfied, the Feynman-Kac theorem now allows us to formally write down the corresponding pre-default partial differential equation (PDE) of the pricing problem. The formal proof is the same as that of Feynman-Kac for the linear case and relies on the stochastic integral term being a martingale and applying Itô's formula twice. Further, we need to assume that the underlying market risk factors are Markov processes with infinitesimal generator $\mathcal{L}_{t}$, that the risk-free rate $r_{t}$ is bounded, and that we have sufficient smoothness of the price function $\bar{V}$. Hence, for $\tau>t$, we get the PDE

$$
\left(\partial_{t}-\tilde{f}_{t}-\lambda_{t}+\mathcal{L}_{t}\right) \bar{V}_{t}-\left(r_{t}-\tilde{f}_{t}\right) H_{t}+\left(\tilde{f}_{t}-\tilde{c}_{t}\right) C_{t}+\lambda_{t} \theta_{t}+\partial_{t} \Pi_{t}=0
$$

with boundary condition

$$
\bar{V}_{T}=0
$$

and where

$$
\partial_{t} \Pi_{t}=\lim _{h \downarrow 0} \mathbb{E}_{t}\left[\frac{\Pi(t, t+h)}{h} \mid \mathcal{F}\right] .
$$

To keep the notation simple, we have suppressed the dependence of $\bar{V}$ on $(C, F)$. If we, furthermore, assume that the underlying risk factors are diffusions and consider deltahedging of the deal value $\bar{V}$ in continuous time, the generator $\mathcal{L}$ can be expanded in terms of first and second order operators

$$
\mathcal{L}_{t} \bar{V}_{t} \triangleq\left(\mathcal{L}_{t}^{1}+\mathcal{L}_{t}^{2}\right) \bar{V}_{t} \triangleq r_{t} H_{t}+\mathcal{L}_{t}^{2} \bar{V}_{t}
$$


As a result, for $\tau>t$, the pricing PDE reduces to

$$
\left(\partial_{t}-\tilde{f}_{t}-\lambda_{t}+\mathcal{L}_{t}^{\tilde{f}}\right) \bar{V}_{t}+\left(\tilde{f}_{t}-\tilde{c}_{t}\right) C_{t}+\lambda_{t} \theta_{t}+\partial_{t} \Pi_{t}=0
$$

where $\mathcal{L}_{t}^{\tilde{f}} \bar{V}_{t} \triangleq \tilde{f}_{t} H_{t}+\mathcal{L}_{t}^{2} \bar{V}_{t}$. Observe that the pre-default PDE no longer depends on the risk-free rate $r_{t}$. This equation may be solved numerically as in Crépey (2012a). On the other hand, if we apply the Feynman-Kac theorem again - this time going from the pre-default PDE to the pricing expectation - and integrate by parts, we arrive at the following result

Theorem 8 (Continuous-time Solution of the General Pricing Equation). If we assume collateral rehypothecation and delta-hedging, we can solve the iterative equations of Theorem 7 in continuous time. We get

$$
\begin{aligned}
\bar{V}_{t}(C ; F)= & C_{t}+\mathbb{E}_{t}^{\tilde{f}}\left[\mathbf{1}_{\{\tau<T\}}\left(\theta_{\tau}(C, \varepsilon)-C_{\tau^{-}}\right) D(t, \tau ; \tilde{f})\right] \\
& +\int_{t}^{T} \mathbb{E}_{t}^{\tilde{f}}\left[\mathbf{1}_{\{s<\tau\}}\left(d \Pi_{s}+d C_{s}-\tilde{c}_{s} C_{s} d s\right) D(t, s ; \tilde{f})\right],
\end{aligned}
$$

where $d \Pi_{s}=\Pi(s, s+d s)$ and the expectations are taken under the pricing measure $\mathbb{Q}^{\tilde{f}}$ for which the underlying risk factors grow at the rate $\tilde{f}$ if no dividend is paid.

Theorem 8 decomposes the deal price $\bar{V}$ into three intuitive terms. The first term is the value of the collateral. The second term is the price of the on-default cash-flow in excess of the collateral, which includes the CVA and DVA of the deal. The last term collects the price of marked-to-market movements in the derivative not followed by an immediate reset of the collateral account as well as the margining costs not included in the value of the collateral. In addition, we see that any dependence on the hedging strategy can be dropped by taking all expectations under the pricing measure $\mathbb{Q}^{\tilde{f}}$.

Finally, we stress that the pricing equation in the above theorem is completely governed by market rates; there is no dependence on a risk-free rate. This confirms our earlier conjecture that the risk-free rate is merely an instrumental variable of our pricing framework and we do not, in fact, need to know the value of such a rate.

\subsection{Numerical Results}

This section provides a numerical study of the valuation framework outlined in the previous sections. We investigate the impact of funding risk on the price of a derivatives deal 
under default risk and collateralization. To this end, we propose a least-squares Monte Carlo algorithm inspired by the simulation methods of Carriere (1996), Longstaff and Schwartz (2001), Tilley (1993), and Tsitsiklis and Van Roy (2001) for pricing Americanstyle options. As the purpose is to understand the fundamental implications of funding risk, we focus on relatively simple deal positions in European call options. However, the Monte Carlo method we propose below can be applied to more complex derivative contracts, including derivativess with bilateral payments.

\subsubsection{Monte Carlo Algorithm}

Recall the recursive structure of the general pricing equation: The deal price depends on the funding decisions, while the optimal funding strategy depends on the price itself. The intimate relationship among the key quantities makes the pricing problem computationally challenging.

We consider $K$ default scenarios during the life of the deal - either obtained by simulation, bootstrapped from empirical data, or assumed in advance. For each first-to-default time $\tau$ corresponding to a default scenario, we compute the price of the deal $\bar{V}$ under collateralization, close-out netting and funding costs. The first step of our simulation method entails simulating a large number of sample paths $N$ of the underlying risk factors $X$. We simulate these paths on the time-grid $\left\{t_{1}, \ldots, t_{m}=T^{*}\right\}$ with step size $\Delta t=t_{j+1}-t_{j}$ from the assumed dynamics of the risk factors. $T^{*}$ is equal to the final maturity $T$ of the deal or the consecutive time-grid point following the first-default time $\tau$, whichever occurs first. For simplicity, we assume the time periods for funding decisions and collateral margin payments coincide with the simulation time grid.

Given the set of simulated paths, we solve the optimal funding strategy recursively in a dynamic programming fashion. Starting one period before $T^{*}$, we compute for each simulated path the optimal funding decision $F$ and the deal price $\bar{V}$ according to the set of backward-inductive equations of Theorem 7. The algorithm then proceeds recursively until time zero. Ultimately, the total price of the deal is computed as the probability weighted average of the individual prices obtained in each of the $K$ default scenarios.

The conditional expectations in the backward-inductive funding equations are approximated by across-path regressions based on least squares estimation similar to Longstaff and Schwartz (2001). We regress the present value of the deal price at time $t_{j+1}$, the adjusted payout cash flow between $t_{j}$ and $t_{j+1}$, the collateral account and funding account 
at time $t_{j}$ on basis functions $\psi$ of realizations of the underlying risk factors at time $t_{j}$ across the simulated paths. To keep notation simple, let us assume that we are exposed to only one underlying risk factor, e.g. a stock price. Extensions to higher dimensions are straightforward. Specifically, the conditional expectations in the iterative equations of Theorem 7, taken under the risk-neutral measure, are equal to

$$
\mathbb{E}_{t_{j}}\left[\Xi_{t_{j}}\left(\bar{V}_{t_{j+1}}\right)\right]=\theta_{t_{j}}^{\prime} \psi\left(X_{t_{j}}\right)
$$

where we have defined $\Xi_{t_{j}}\left(\bar{V}_{t_{j+1}}\right) \triangleq D\left(t_{j}, t_{j+1}\right) \bar{V}_{t_{j+1}}(C ; F)+\bar{\pi}\left(t_{j}, t_{j+1} ; C\right)-C_{t_{j}}-H_{t_{j}}$. Note the $C_{t_{j}}$ term drops out if rehypothecation is not allowed. The usual least-squares estimator of $\theta$ is then given by

$$
\hat{\theta}_{t_{j}} \triangleq\left[\psi\left(X_{t_{j}}\right) \psi\left(X_{t_{j}}\right)^{\prime}\right]^{-1} \psi\left(X_{t_{j}}\right) \Xi_{t_{j}}\left(\bar{V}_{t_{j+1}}\right) .
$$

Orthogonal polynomials such as Chebyshev, Hermite, Laguerre, and Legendre may all be used as basis functions for evaluating the conditional expectations. We find, however, that simple power series are quite effective and that the order of the polynomials can be kept relatively small. In fact, linear or quadratic polynomials, i.e. $\psi\left(X_{t_{j}}\right)=\left(\mathbf{1}, X_{t_{j}}, X_{t_{j}}^{2}\right)^{\prime}$, are often enough.

Further complexities are added, as the dealer may - realistically - decide to hedge the full deal price $\bar{V}$. Now, the hedge $H$ itself depends on the funding strategy through $\bar{V}$, while the optimal funding decision depends on the hedging strategy. This added recursion requires that we solve the optimal funding and hedging strategies simultaneously. For example, if the dealer applies a delta-hedging strategy

$$
H_{t_{j}}=\left.\frac{\partial \bar{V}}{\partial X}\right|_{t_{j}} X_{t_{j}} \approx \frac{\bar{V}_{t_{j+1}}-\Delta t_{j}\left(1+\tilde{f}_{t_{j}}\right) \bar{V}_{t_{j}}}{X_{t_{j+1}}-\Delta t_{j}\left(1+\tilde{f}_{t_{j}}\right) X_{t_{j}}} X_{t_{j}}
$$

we obtain, in the case of rehypothecation, the following system of non-linear equations

$$
\left\{\begin{array}{l}
F_{t_{j}}-\frac{P_{t_{j}}^{\tilde{f}}\left(t_{j+1}\right)}{P_{t_{j}}\left(t_{j+1}\right)} \mathbb{E}_{t_{j}}\left[\Xi_{t_{j}}\left(\bar{V}_{t_{j+1}}\right)\right]=0 \\
H_{t_{j}}-\frac{\bar{V}_{t_{j+1}}-\Delta t_{j}\left(1+\tilde{f}_{t_{j}}\right) \bar{V}_{t_{j}}}{X_{t_{j+1}}-\Delta t_{j}\left(1+\tilde{f}_{t_{j}}\right) X_{t_{j}}} X_{t_{j}}=0 \\
\bar{V}_{t_{j}}=F_{t_{j}}+C_{t_{j}}+H_{t_{j}}
\end{array}\right.
$$

where all matrix operations are on an element-by-element basis. An analogous result holds when rehypothecation of the posted collateral is forbidden. 
Each period and for each simulated path, we find the funding and hedging decisions by solving this system of equations, given the optimal funding and hedging strategies for all future periods until the end of the deal. We apply a simple Newton-Raphson method to solve the system of non-linear equations numerically, but instead of using the exact Jacobian, we approximate it by finite differences. As initial guess, we use the Black-Scholes delta position

$$
H_{t_{j}}^{0}=\Delta_{t_{j}}^{B S} X_{t_{j}}
$$

The convergence is quite fast and only a small number of iterations are needed in practice. Finally, if the dealer for some reason decides to only hedge the risk-free price of the deal, i.e. the classic derivative price $V$, the pricing problem collapses to a much simpler one. The hedge $H$ no longer depends on the funding decision and can be computed separately and the numerical solution of the non-linear equation system can be avoided altogether.

\subsubsection{Simulation Experiments}

We now report numerical results on the impact of funding for both a long and a short position in a European call option. We assume that the underlying stock evolves according to a geometric Brownian motion. The risk-free interest rate $r$ is $100 \mathrm{bps}$, the volatility $\sigma$ is $25 \%$, and the current price of the underlying is $S_{0}=100$. The European call option is in-the-money and has strike $K=80$. The maturity $T$ of the deal is 3 years and we assume that the investor delta-hedges the deal according to (4.22). The usual Black-Scholes price of the deal is 28.9 .

We consider two simple discrete probability distributions of default. Both parties of the deal are considered default risky but can only default at year 1 or at year 2 . The localized joint default probabilities are provided in the matrices below. The rows denote the default time of the investor, while the columns denote the default times of the counterparty. For example, in matrix $D_{\text {low }}$ the event $\left(\tau_{I}=2 y r, \tau_{C}=1 y r\right)$ has a $3 \%$ probability and the first-to-default time is 1 year. Simultaneous defaults are introduced and we determine the close-out amount by a random draw from a uniform distribution. If the random number is above 0.5 , we compute the close-out as if the counterparty defaulted first, and vice versa.

For the first default distribution, we have a low dependence between the default risk 
of the counterparty and the default risk of the investor

$$
\begin{aligned}
& 1 y r \text { 2yr n.d. } \\
& D_{\text {low }}=\begin{array}{l}
1 y r \\
2 y r \\
n . d .
\end{array}\left(\begin{array}{lll}
0.01 & 0.01 & 0.03 \\
0.03 & 0.01 & 0.05 \\
0.07 & 0.09 & 0.70
\end{array}\right), \quad \tau_{K}\left(D_{\text {low }}\right)=0.21
\end{aligned}
$$

where $n . d$. means no default and $\tau_{K}$ denotes the rank correlation as measured by Kendall's tau. In the second case, we have a high dependence between the two parties' default risk

$$
\begin{aligned}
& 1 y r \text { 2yr n.d. } \\
& D_{\text {high }}=\begin{array}{l}
1 y r \\
2 y r \\
\text { n.d. }
\end{array}\left(\begin{array}{ccc}
0.09 & 0.01 & 0.01 \\
0.03 & 0.11 & 0.01 \\
0.01 & 0.03 & 0.70
\end{array}\right), \quad \tau_{K}\left(D_{\text {high }}\right)=0.83
\end{aligned}
$$

Note also that the distributions are skewed in the sense that the counterparty has a higher default probability than the investor. The loss given default is $50 \%$ for both the investor and the counterparty and the loss on any posted collateral is considered the same. The collateral rates are chosen to be equal to the risk free rate. We assume that the collateral account is equal to the risk-free price of the deal at each margin date, i.e. $C_{t}=V_{t}$. This is reasonable as the dealer and client will be able to agree on this price, in contrast to $\bar{V}_{t}$ due to asymmetric information. Also, choosing the collateral this way has the added advantage that the collateral account $C$ works as a control variate, reducing the variance of the least-squares Monte Carlo estimator of the deal price.

The recursive structure makes the pricing problem particularly demanding in terms of computational time, so we are forced to choose a relatively small number of sample paths. We use 1,000 sample paths but, fortunately, the presence of collateral mitigates too large errors. In Tables 4.2-4.3 we conduct a ceteris paribus analysis of funding risk under counterparty credit risk and collateralization. Specifically, we investigate how the value of a deal changes for different values of the borrowing (lending) rate $f^{+}\left(f^{-}\right)$while keeping the lending (borrowing) rate fixed to $100 \mathrm{bps}$. When both funding rates equal to $100 \mathrm{bps}$ the deal is funded at the risk-free rate and we are in the classical derivatives pricing setting.

To provide some ball-park figures on the effect of funding risk, we first look at the case without default risk and without collateralization of the deal. Table 4.1 reports the results of this preliminary step. In absolute values, one observes a symmetric impact of 
funding - as expected - between the long and short cases when no default risk is present. Also, notice for symmetric funding rates equal to the risk-free rate, ie. $f^{+}=f^{-}=r$, we obtain a price equal to the Black-Scholes price within Monte Carlo noise.

Table 4.1: Price impact of funding without default risk

\begin{tabular}{lcc}
\hline \hline & \multicolumn{2}{c}{ Default-free } \\
\cline { 2 - 3 } Funding $^{\mathrm{a}}$ & Long & Short \\
\hline Borrowing rate $f^{+}$ & & \\
$0 \mathrm{bps}$ & $29.92(0.38)$ & $-26.89(0.29)$ \\
$100 \mathrm{bps}$ & $29.05(0.25)$ & $-29.05(0.25)$ \\
$200 \mathrm{bps}$ & $28.57(0.26)$ & $-31.46(0.23)$ \\
$300 \mathrm{bps}$ & $27.94(0.43)$ & $-33.68(0.25)$ \\
$400 \mathrm{bps}$ & $27.61(0.51)$ & $-35.79(0.40)$ \\
\hline Lending rate $f^{-}$ & & \\
0 bps & $26.89(0.29)$ & $-29,96(0.40)$ \\
$100 \mathrm{bps}$ & $29.05(0.25)$ & $-29.05(0.25)$ \\
$200 \mathrm{bps}$ & $31.47(0.23)$ & $-28.51(0.29)$ \\
$300 \mathrm{bps}$ & $33.69(0.26)$ & $-27.88(0.47)$ \\
$400 \mathrm{bps}$ & $35.70(0.40)$ & $-27.50(0.57)$ \\
\hline \hline
\end{tabular}

Next, we switch on credit risk and consider collateralized deals. Table 4.2 reports the impact of changing funding rates for a call position when the posted collateral may not be used for funding the deal, i.e. rehypothecation is not allowed. First, for the long position, increasing the lending rate $f^{-}$while keeping the borrowing rate $f^{+}$fixed causes an increase in the deal value. On the other hand, an increase in the borrowing rate while fixing the lending rate, decreases the value of the short position, i.e. the negative exposure of the investor increases. As a call option is just a one-sided contract, increasing the borrowing rate for a long position only has a minor impact. To hedge a long call, the investor goes short in a delta position of the underlying asset and invests excess cash in the treasury at $f^{-}$. Correspondingly, to hedge the short position, the investor enters a long delta position in the stock and finances it by borrowing cash from the treasury at $f^{+}$, so changing the lending rate only has a small effect on the deal value. Finally, due 
Table 4.2: Price impact of funding with default risk and collateralization

\begin{tabular}{|c|c|c|c|c|}
\hline \multirow[b]{2}{*}{ Funding $^{\mathrm{a}}$} & \multicolumn{2}{|c|}{ Default risk, low ${ }^{b}$} & \multicolumn{2}{|c|}{ Default risk, high ${ }^{\mathrm{c}}$} \\
\hline & Long & Short & Long & Short \\
\hline \multicolumn{5}{|c|}{ Borrowing rate $f^{+}$} \\
\hline $0 \mathrm{bps}$ & $29.04(0.14)$ & $-26.33(0.18)$ & $29.33(0.24)$ & $-26.73(0.35)$ \\
\hline $100 \mathrm{bps}$ & $28.70(0.15)$ & $-28.72(0.15)$ & $29.06(0.21)$ & $-29.07(0.21)$ \\
\hline $200 \mathrm{bps}$ & $28.04(0.26)$ & $-31.19(0.29)$ & $28.50(0.25)$ & $-31.47(0.23)$ \\
\hline $300 \mathrm{bps}$ & $27.33(0.43)$ & $-33.65(0.46)$ & $27.88(0.29)$ & $-33.86(0.38)$ \\
\hline $400 \mathrm{bps}$ & $26.59(0.64)$ & $-36.56(0.77)$ & $27.26(0.36)$ & $-36.70(0.71)$ \\
\hline \multicolumn{5}{|c|}{ Lending rate $f^{-}$} \\
\hline $0 \mathrm{bps}$ & $26.50(0.17)$ & $-29.11(0.12)$ & $26.96(0.33)$ & $-29.37(0.24)$ \\
\hline $100 \mathrm{bps}$ & $28.70(0.15)$ & $-28.72(0.15)$ & $29.06(0.21)$ & $-29.07(0.21)$ \\
\hline $200 \mathrm{bps}$ & $30.98(0.28)$ & $-28.09(0.28)$ & $31.27(0.22)$ & $-28.51(0.25)$ \\
\hline 300 bps & $33.17(0.44)$ & $-27.38(0.45)$ & $33.44(0.36)$ & $-27.90(0.29)$ \\
\hline $400 \mathrm{bps}$ & $35.67(0.69)$ & $-26.63(0.66)$ & $35.70(0.61)$ & $-27.27(0.36)$ \\
\hline
\end{tabular}

Standard errors of the price estimates are given in parentheses.

a Ceteris paribus changes in one funding rate while keeping the other fixed to 100 bps.

b Based on the joint default distribution $D_{\text {low }}$ with low dependence.

c Based on the joint default distribution $D_{\text {high }}$ with high dependence.

to the presence of collateral, we observe an almost similar price impact of funding under the two different default distributions $D_{\text {low }}$ and $D_{\text {high }}$.

Finally, assuming cash collateral, we consider the case of rehypothecation and allow the investor and counterparty to use any posted collateral as a funding source. If the collateral is posted to the investor, this means it effectively reduces his costs of funding the delta-hedging strategy. As the payoff of the call is one-sided, the investor only receives collateral when he holds a long position in the call option. But as he hedges this position by short-selling the underlying stock and lending the excess cash proceeds, the collateral adds to his cash lending position and increases the funding benefit of the deal. Analogously, if the investor has a short position, he posts collateral to the counterparty and a higher borrowing rate would increase his costs of funding the collateral he has to post as well as his delta-hedge position. Table 4.3 reports the results for the short and long positions in the call option.

This shows that funding asymmetry matters even under full collateralization when 
Table 4.3: Price impact of funding with default risk, collateralization, and rehypothecation

\begin{tabular}{lccccc}
\hline \hline & \multicolumn{2}{c}{ Default risk, low } & & \multicolumn{2}{c}{ Default risk, high } \\
\cline { 2 - 3 } \cline { 5 - 6 } Funding $^{\mathrm{c}}$ & Long & Short & & Long & Short \\
\hline Borrowing rate $f^{+}$ & & & & & \\
$\quad 0 \mathrm{bps}$ & $29.39(0.15)$ & $-25.70(0.24)$ & & $29.69(0.23)$ & $-26.09(0.41)$ \\
$100 \mathrm{bps}$ & $28.72(0.15)$ & $-28.77(0.15)$ & & $29.09(0.23)$ & $-29.11(0.22)$ \\
$200 \mathrm{bps}$ & $28.33(0.27)$ & $-32.07(0.32)$ & & $28.77(0.22)$ & $-32.35(0.26)$ \\
$300 \mathrm{bps}$ & $28.16(0.22)$ & $-35.71(0.62)$ & & $28.52(0.18)$ & $-35.92(0.55)$ \\
$400 \mathrm{bps}$ & $27.67(0.58)$ & $-39.62(0.94)$ & & $28.25(0.31)$ & $-39.77(0.91)$ \\
\hline Lending rate $f^{-}$ & & & & & \\
$0 \mathrm{bps}$ & $25.65(0.24)$ & $-29.43(0.11)$ & & $26.08(0.41)$ & $-29.71(0.23)$ \\
$100 \mathrm{bps}$ & $28.72(0.15)$ & $-28.77(0.15)$ & & $29.09(0.23)$ & $-29.11(0.22)$ \\
$200 \mathrm{bps}$ & $32.02(0.33)$ & $-28.37(0.24)$ & & $32.34(0.26)$ & $-28.77(0.19)$ \\
$300 \mathrm{bps}$ & $35.65(0.63)$ & $-28.04(0.35)$ & & $35.91(0.55)$ & $-28.47(0.20)$ \\
$400 \mathrm{bps}$ & $39.57(0.96)$ & $-27.64(0.58)$ & & $39.75(0.92)$ & $-28.18(0.31)$ \\
\hline \hline
\end{tabular}

Standard errors of the price estimates are given in parentheses.

a Ceteris paribus changes in one funding rate while keeping the other fixed to 100 bps.

b Based on the joint default distribution $D_{\text {low }}$ with low dependence.

c Based on the joint default distribution $D_{\text {high }}$ with high dependence.

there is no repo market for the underlying stock. In practice, however, the dealer cannot hedge a long call by shorting a stock he does not own. Instead, he would first borrow the stock in a repo transaction and then sell it in the spot market. Similarly, to enter the long delta position needed to hedge a short call, the dealer could finance the purchase by lending the stock in a reverse repo transaction. Effectively, the delta position in the underlying stock would be funded at the prevailing repo rate. Thus, once the delta hedge has to be executed through the repo market, there is no funding valuation adjustment (meaning any dependence on the funding rate $\tilde{f}$ drops out) given the deal is fully collateralized, but the underlying asset still grows at the repo rate. If there is no credit risk, this would leave us with the result of Piterbarg (2010). However, if the deal is not fully collateralized or the collateral cannot be rehypothecated, funding costs enter the picture even when there is a repo market for the underlying stock. In the following section we extend the theoretical framework to address these real-world issues. 


\subsection{Extensions}

So far, we have more or less silently made the assumption that the dealer hedges the derivatives position by trading in the spot market of the underlying asset(s). Nonetheless, to be in business, the dealer might decide or even be forced to implement a hedging strategy that involves trading the underlying assets through stock-lending or repo markets or by entering other derivatives positions, e.g., (synthetic) forward contracts on the underlying risk factors. As a result, the dealer may incur additional costs or revenues which we obviously need to include when pricing the deal. To address this issue, we introduce two general adaptive processes: $h_{t}^{-}(T)$ is the rate of hedging revenue for lending risky assets from time $t$ to $T$, while $h_{t}^{+}(T)$ is the hedging cost rate for asset borrowing. The corresponding effective hedging rate is defined as

$$
\tilde{h}_{t}(T) \triangleq h_{t}^{-}(T) \mathbf{1}_{\left\{H_{t}<0\right\}}+h_{t}^{+}(T) \mathbf{1}_{\left\{H_{t}>0\right\}} .
$$

For example, if the dealer hedges in the stock-lending or repo market, we can apply the quoted repo rate as the hedging rate. Another example is hedging by trading in collateralized markets, i.e. markets where only collateralized financial contracts are quoted. The money market falls in this category and contracts traded in this market are collateralized on a daily basis at the over-night rate. So, if the hedging strategy implies trading directly in the money market, the effective hedging cost is simply given by the collateral rate itself.

If we assume the hedging strategy is implemented on the same time-grid as the funding procedure of the deal, we can sum both the funding and hedging costs in a single term. This leads us to redefine $\varphi$ in (4.13) so it explicitly takes the dependence on the hedging strategy into account

$$
\begin{aligned}
\varphi(t, T \wedge \tau ; F, H) \triangleq & \sum_{j=1}^{m-1} \mathbf{1}_{\left\{t \leqslant t_{j}<(T \wedge \tau)\right\}} D\left(t, t_{j}\right) F_{t_{j}}\left(1-\frac{P_{t_{j}}\left(t_{j+1}\right)}{P_{t_{j}}^{\tilde{f}}\left(t_{j+1}\right)}\right) \\
& -\sum_{j=1}^{m-1} \mathbf{1}_{\left\{t \leqslant t_{j}<(T \wedge \tau)\right\}} D\left(t, t_{j}\right) H_{t_{j}}\left(\frac{P_{t_{j}}\left(t_{j+1}\right)}{P_{t_{j}}^{\tilde{f}}\left(t_{j+1}\right)}-\frac{P_{t_{j}}\left(t_{j+1}\right)}{P_{t_{j}}\left(t_{j+1}\right)}\right),
\end{aligned}
$$

where the zero-coupon hedging bond is defined as $P_{t}^{\tilde{h}}(T) \triangleq\left[1+(T-t) \tilde{h}_{t}(T)\right]^{-1}$. Moreover, if we take the continuous-time limit, assuming that rehypothecation is allowed, we obtain

$$
\begin{aligned}
\varphi(t, T \wedge \tau ; F)= & \int_{t}^{T \wedge \tau}\left(r_{s}-\tilde{f}_{s}\right)\left[\bar{V}_{s}(C, F)-C_{s}\right] D(t, s) d s \\
& -\int_{t}^{T \wedge \tau}\left(r_{s}-\tilde{h}_{s}\right) H_{s} D(t, s) d s,
\end{aligned}
$$


which assumes funding and hedging of the deal takes place in continuous time. For the case when rehypothecation is forbidden, the result can be obtained analogously. We can now repeat the calculations of Section 4.3.2 to obtain the pre-default PDE for the continuous-time pricing problem in the case of hedging costs, which is given by

$$
\left(\partial_{t}-\tilde{f}_{t}-\lambda_{t}+\mathcal{L}_{t}\right) \bar{V}_{t}-\left(r_{t}-\tilde{h}_{t}\right) H_{t}+\left(\tilde{f}_{t}-\tilde{c}_{t}\right) C_{t}+\lambda_{t} \theta_{t}+\partial_{t} \Pi_{t}=0, \quad \tau>t,
$$

with boundary condition (4.17). Assuming diffusive underlying risk factors and deltahedging, this collapses to the PDE

$$
\left(\partial_{t}-\tilde{f}_{t}-\lambda_{t}+\mathcal{L}_{t}^{\tilde{h}}\right) \bar{V}_{t}+\left(\tilde{f}_{t}-\tilde{c}_{t}\right) C_{t}+\lambda_{t} \theta_{t}+\partial_{t} \Pi_{t}=0, \quad \tau>t,
$$

where the generator is defined as $\mathcal{L}_{t}^{\tilde{h}} \bar{V}_{t} \triangleq \tilde{h}_{t} H_{t}+\mathcal{L}_{t}^{2} \bar{V}_{t}$. Again, we could solve this equation numerically, but we choose to apply the reasoning of Section 4.3 .2 to reach a similar result of Theorem 8 when the dealer needs to implement the hedging strategy by trading in the derivatives market and not just the spot market.

Corollary 1 (Continuous-time Solution with Hedging Costs). If we assume collateral rehypothecation and delta-hedging, we can solve the iterative equations of Theorem 7 in continuous time when the hedging strategy is implemented by trading on a derivative market where the effective hedging rate is $\tilde{h}$. We obtain

$$
\begin{aligned}
\bar{V}_{t}(C ; F)= & C_{t}+\mathbb{E}_{t}^{\tilde{h}}\left[\mathbf{1}_{\{\tau<T\}}\left(\theta_{\tau}(C, \varepsilon)-C_{\tau^{-}}\right) D(t, \tau ; \tilde{f})\right] \\
& +\int_{t}^{T} \mathbb{E}_{t}^{\tilde{h}}\left[\mathbf{1}_{\{u<\tau\}}\left(d \Pi_{u}+d C_{u}-\tilde{c}_{u} C_{u} d u\right) D(t, u ; \tilde{f})\right]
\end{aligned}
$$

where the expectations are taken under a pricing measure $\mathbb{Q}^{\tilde{h}}$ for which the underlying risk factors grow at the rate $\tilde{h}$.

Analogous to the case of hedging in the spot market, we incorporate the additional hedging costs by altering the drift of the price processes of the underlying risk factors. Moreover, by handling hedging costs via a change of measure, we observe that the explicit dependence on $H_{t}$ disappears from the pricing equation.

\subsection{Conclusion}

We have developed an arbitrage-free framework for consistent valuation of OTC derivative trades with collateralization, counterparty credit risk, and funding costs. Based on the 
risk-neutral pricing principle, we derive a general pricing equation where CVA, DVA, and FVA are introduced by simply modifying the payout cash-flows of the deal. The pricing problem is highly non-linear and recursive in line with the recent findings of Crépey (2011, 2012a,b). The price of the deal depends on the trader's funding strategy, while to determine the optimal funding strategy we need to know the deal price itself. This means that FVA is not generally an additive adjustment, let alone a discounting spread, as commonly assumed by market participants.

Our valuation framework addresses common market practices of ISDA and CSA governed deals without restrictive assumptions on collateral margin payments and close-out netting rules. In particular, we allow for asymmetric collateral and funding rates as observed in practice. The introduction of funding risk breaks the bilateral nature of the deal price. The particular funding policy chosen by the client is not known to the dealer, and vice versa. As a result, the price of the deal would be different to the two parties. Theoretically, this should mean that the parties would never close the deal, but in reality dealers say this was the key factor driving bid-ask spreads wider during the crisis. Finally, we show that the general pricing equation can be cast as a set of backward-iterative equations. These equations can be solved recursively by means of least-squares Monte Carlo and we propose such a simulation algorithm. Our numerical results confirm that funding risk does have a non-trivial impact on the deal price. 



\section{Bibliography}

Abadir, K. and M. Rockinger (2003). Density functionals with an option-pricing application. Econometric Theory 19(5), 778-811.

Abate, J. and W. Whitt (1995). Numerical inversion of laplace transforms of probability distributions. ORSA Journal on computing 7(1), 36-43.

Abramowitz, M. and I. A. Stegun (1987). Handbook of mathematical functions. Dover publications, INC. New York.

Aït-Sahalia, Y. (2002). Maximum likelihood estimation of discretely sampled diffusions: A closed-form approximation approach. Econometrica 70(1), 223-262.

Ait-Sahalia, Y. and J. Yu (2006). Saddlepoint approximations for continuous-time markov processes. Journal of Econometrics 134(2), 507 - 551.

Alòs, E. (2012). A decomposition formula for option prices in the heston model and applications to option pricing approximation. Finance and Stochastics 16 (3), 403-422.

Alòs, E., J. Léon, and J. Vives (2007). On the short-time behavior of the implied volatility for jump-diffusion models with stochastic volatility. Finance and Stochastics $11(4)$, $571-589$.

Andersen, L. and J. Andreasen (2002). Volatile volatilities. RISK 15(12), 163-163.

Andersen, L. and V. Piterbarg (2011). Interest Rate Modeling, Volume I: Foundations and Vanilla Models. Atlantic Financial Press. 
Antonelli, F. and S. Scarlatti (2009). Pricing options under stochastic volatility: a power series approach. Finance and Stochastics 13(2), 269-303.

Bakshi, G., C. Cao, and Z. Chen (1997). Empirical performance of alternative option pricing models. Journal of Finance 52(5), 2003-2049.

Ball, C. and A. Roma (1994). Stochastic volatility option pricing. Journal of Financial and Quantitative Analysis 29(4), 589-607.

Bank for International Settlements (2013, May). Statistical release: OTC derivatives statistics at end-December 2012. Technical report.

Barndorff-Nielsen, O. (1998). Processes of normal inverse gaussian type. Finance and Stochastics 2(1), 41-68.

Barndorff-Nielsen, O. and N. Shephard (2001). Non-gaussian ornstein-uhlenbeck-based models and some of their uses in financial economics. Journal of the Royal Statistical Society 63(2), 167-241.

Barndorff-Nielsen, O. and N. Shephard (2003). Integrated ou processes and non-gaussian ou-based stochastic volatility models. Scandinavian Journal of statistics 30(2), 277295.

Benaim, S. and P. Friz (2008). Smile asymptotics ii: models with known moment generating functions. Journal of Applied Probability 45(1), 16-32.

Benaim, S. and P. Friz (2009). Regular variation and smile asymptotics. Mathematical Finance 19(1), 1-12.

Benaim, S., P. Friz, and R. Lee (2008). On black-scholes implied volatility at extreme strikes. Frontiers in Quantitative Finance: Volatility and Credit Risk Modeling, 19-45.

Benhamou, E., E. Gobet, and M. Miri (2009). Smart expansion and fast calibration forjump-diffusions. Finance and Stochastics 13(4), 563-589.

Berestycki, H., I. Florent, et al. (2004). Computing the implied volatility in stochastic volatility models. Communications on Pure and Applied Mathematics 57(10), 13521373. 
Bielecki, T. and M. Rutkowski (2002). Credit Risk: Modeling, Valuation and Hedging. Springer Finance, Berlin.

Black, F. and M. Scholes (1973). The pricing of options and corporate liabilities. The Journal of Political Economy 81, 637-654.

Boyarchenko, S. and S. Levendorskii (2002). Non-Gaussian Merton-Black-Scholes Theory. World Scientific.

Brigo, D. and I. Bakkar (2009). Accurate counterparty risk valuation for energycommodities swaps. Energy Risk March.

Brigo, D. and A. Capponi (2008). Bilateral counterparty risk with application to CDSs. Working Paper, 241-268.

Brigo, D., A. Capponi, and A. Pallavicini (2011). Arbitrage-free bilateral counterparty risk valuation under collateralization and re-hypothecation with application to CDS. Mathematical Finance. (Forthcoming).

Brigo, D., A. Capponi, A. Pallavicini, and V. Papatheodorou (2011). Collateral margining in arbitrage-free counterparty valuation adjustment including re-hypotecation and netting. Working Paper.

Brigo, D. and K. Chourdakis (2009). Counterparty risk for credit default swaps: Impact of spread volatility and default correlation. International Journal of Theoretical and Applied Finance 12 (07), 1007-1026.

Brigo, D. and M. Masetti (2005). Risk neutral pricing of counterparty risk. In M. Pykhtin (Ed.), Counterparty Credit Risk Modeling: Risk Management, Pricing and Regulation. Risk Books.

Brigo, D., M. Morini, and M. Tarenghi (2011). Equity return swap valuation under counterparty risk. In T. Bielecki, D. Brigo, and F. Patras (Eds.), Credit Risk Frontiers: Sub- prime crisis, Pricing and Hedging, CVA, MBS, Ratings and Liquidity, pp. 457484. Wiley.

Brigo, D. and A. Pallavicini (2007). Counterparty risk under correlation between default and interest rates. In J. Miller, D. Edelman, and J. Appleby (Eds.), Numerical Methods for Finance. Chapman Hall. 
Brigo, D., A. Pallavicini, and V. Papatheodorou (2011). Arbitrage-free valuation of bilateral counterparty risk for interest-rate products: impact of volatilities and correlations. International Journal of Theoretical and Applied Finance 14 (6), 773-802.

Broadie, M., M. Chernov, and M. Johannes (2007). Model specification and risk premia: Evidence from futures options. Journal of Finance 62(3), 1453-1490.

Broadie, M. and A. Jain (2008). The effect of jumps and discrete sampling on volatility and variance swaps. International Journal of Theoretical and Applied Finance 11(08), $761-797$.

Burgard, C. and M. Kjaer (2011a). In the balance. Risk Magazine October.

Burgard, C. and M. Kjaer (2011b). Partial differential equation representations of derivatives with counterparty risk and funding costs. The Journal of Credit Risk 7 (3), 1-19.

Carr, P. (2000). Deriving derivatives of derivative securities. Journal of Computational Finance 4(2), 5-29.

Carr, P., H. Geman, D. Madan, and M. Yor (2002). The fine structure of asset returns: an empirical investigation. Journal of Business 75(2), 305-332.

Carr, P., H. Geman, D. Madan, and M. Yor (2003). Stochastic volatility for lévy processes. Mathematical Finance 13(3), 345-382.

Carr, P., H. Geman, D. Madan, and M. Yor (2005). Pricing options on realized variance. Finance and Stochastics 9(4), 453-475.

Carr, P. and D. Madan (1999). Option valuation using the fast fourier transform. Journal of Computational Finance 2(4), 61-73.

Carriere, J. (1996). Valuation of the early-exercise price for options using simulations and nonparametric regression. Insurance: Mathematics and Economics 19(1), 19-30.

Castagna, A. (2011). Funding, liquidity, credit and counterparty risk: Links and implications. Working Paper.

Chernov, M., A. Ronald G., E. Ghysels, and G. Tauchen (2003). Alternative models for stock price dynamics. Journal of Econometrics 116(1), 225-257. 
Cherubini, U. (2005). Counterparty risk in derivatives and collateral policies: the replicating portfolio approach. In L. Tilman (Ed.), ALM of Financial Institutions. Institutional Investor Books.

Cont, R. and T. Kokholm (2011). A consistent pricing model for index options and volatility derivatives. Mathematical Finance (Forthcoming).

Cont, R. and P. Tankov (2004). Financial Modelling with Jump Processes. Chapman \& Hall.

Cox, J., J. Ingersoll, and S. Ross (1985). A theory of the term structure of interest rates. Econometrica, 385-407.

Crépey, S. (2011). A BSDE approach to counterparty risk under funding constraints. Working Paper.

Crépey, S. (2012a). Bilateral counterparty risk under funding constraints - Part I: Pricing. Forthcoming in Mathematical Finance.

Crépey, S. (2012b). Bilateral counterparty risk under funding constraints - Part II: CVA. Forthcoming in Mathematical Finance.

Crump, K. (1976). Numerical inversion of laplace transforms using a fourier series approximation. Journal of the ACM 23(1), 89-96.

Dormand, J. and P. Prince (1980). A family of embedded runge-kutta formulae. Journal of Computational and Applied Mathematics 6(1), 19-26.

Drimus, G. (2011). Closed-form convexity and cross-convexity adjustments for heston prices. Quantitative Finance 11(8), 1137-1149.

Drimus, G. (2012). Options on realized variance by transform methods: a non-affine stochastic volatility model. Quantitative Finance 12(11), 1679-1694.

Dubner, H. and J. Abate (1968). Numerical inversion of laplace transforms by relating them to the finite fourier cosine transform. Journal of the ACM 15(1), 115-123.

Duffie, D., D. Filipovic, and W. Schachermayer (2003). Affine processes and applications in finance. The Annals of Applied Probability 13(3), 984-1053. 
Duffie, D., J. Pan, and K. Singleton (2000). Transform analysis and asset pricing for affine jump-diffusions. Econometrica 68(6), 1343-1376.

Dufresne, D. (2001). The integrated square-root process. Working Paper, University of Melbourne.

Eberlein, E. and U. Keller (1995). Hyperbolic distributions in finance. Bernoulli 1, 281-299.

Egorov, A., H. Li, and Y. Xu (2003). Maximum likelihood estimation of timeinhomogeneous diffusions. Journal of Econometrics 114(1), 107-139.

Embrechts, P., C. Klüppelberg, and T. Mikosch (1997). Modelling extremal events for insurance and finance. Springer Berlin.

Eraker, B. (2004). Do stock prices and volatility jump? Reconciling evidence from spot and option prices. Journal of Finance 59(3), 1367-1404.

Eraker, B., M. Johannes, and N. Polson (2003). The impact of jumps in volatility and returns. Journal of Finance 58(3), 1269-1300.

Feller, W. (1974). An Introduction to Probability Theory and Its Applications, Volume 2. John Wiley \& Sons.

Filipovic, D., E. Mayerhofer, and P. Schneider (2011). Density approximations for multivariate affine jump-diffusion processes. Swiss Finance Institute Research Paper No. 11-20.

Forde, M. (2008). The small-time behavior of diffusion and time-changed diffusion processes on the line. Unpublished Manuscript.

Forde, M., A. Jacquier, and R. Lee (2012). The small-time smile and term structure of implied volatility under the heston model. SIAM Journal on Financial Mathematics 3(1), 690-708.

Forde, M., A. Jacquier, and A. Mijatovic (2010). Asymptotic formulae for implied volatility in the heston model. Royal Society of London. Proceedings A. Mathematical, Physical and Engineering Sciences 466(2124), 3593-3620. 
Fouque, J., G. Papanicolaou, and K. Sircar (2000). Derivatives in Financial Markets with Stochastic Volatility. Cambridge University Press.

Fries, C. (2010). Discounting revisited: Valuation under funding, counterparty risk and collateralization. Working Paper.

Gatheral, J. (2006). The Volatility Surface: A Practitioner's Guide. Wiley.

Glasserman, P. and K. Kim (2009). Saddlepoint approximations for affine jump-diffusion models. Journal of Economic Dynamics and Control 33(1), 15-36.

Gregory, J. (2009). Being two faced over counterparty credit risk. Risk Magazine 22, $86-90$.

Gulisashvili, A. (2009). Asymptotic formulas with error estimates for call pricing functions and the implied volatility at extreme strikes. Working paper.

Gulisashvili, A. (2012). Asymptotic equivalence in lee's moment formulas for the implied volatility, asset price models without moment explosions, and piterbarg's conjecture. International Journal of Theoretical and Applied Finance (IJTAF) 15(03), 12-46.

Hagan, P., D. Kumar, A. Lesniewski, and D. Woodward (2002). Managing smile risk. Willmot Magazine, 84-108.

Heston, S. (1993). A closed-form solution for options with stochastic volatility with applications to bond and currency options. Review of Financial Studies 6(2), 327-343.

Hoffmann-Jørgensen, J. (1994). Probability with a view toward statistics. Chapman \& Hall.

Hull, J. and A. White (1987). The pricing of options on assets with stochastic volatilities. The Journal of Finance, 281-300.

Hull, J. and A. White (2012). The FVA debate. Risk Magazine 8.

ISDA (2009). ISDA close-out amount protocol. Working Paper.

Iseger, P. (2006). Numerical transform inversion using gaussian quadrature. Probability in the Engineering and Informational Sciences 20(1), 1-44.

Itkin, A. (2005). Pricing options with vg model using fft. Unpublished Manuscript. 
Itkin, A. and P. Carr (2010). Pricing swaps and options on quadratic variation under stochastic time change models - discrete observations case. Review of Derivatives Research 13(2), 141-176.

Jäckel, P. (2009). A singular variance gamma expansion. Unpublished Manuscript.

Jacod, J. and A. Shiryaev (2003). Limit Theorems for Stochastic Processes. Springer New York.

Kallsen, J. (2006). A Didactic Note on Affine Stochastic Volatility Models. From stochastic calculus to mathematical finance, 343-368.

Kallsen, J. and J. Muhle-Karbe (2010). Exponentially affine martingales, affine measure changes and exponential moments of affine processes. Stochastic Processes and their Applications 120, 163-181.

Kallsen, J. and A. Shiryaev (2002). The Cumulant Process and Esscher's Change of Measure. Finance and Stochastics 6(4).

Këllezi, E. and N. Webber (2004). Valuing bermudan options when asset returns are lévy processes. Quantitative Finance 4 (1), 87-100.

Korevaar, J. (2004). Tauberian Theory: A Century of Developments. Springer.

Kristensen, D. and A. Mele (2011). Adding and subtracting black-scholes: a new approach to approximating derivative prices in continuous-time models. Journal of financial economics 102(2), 390-415.

Larsson, K. (2012). General approximation schemes for option prices in stochastic volatility models. Quantitative Finance 12(6), 873-891.

Lee, R. (2001). Implied and local volatilities under stochastic volatility. International Journal of Theoretical and Applied Finance 4 (1), 45-89.

Lee, R. W. (2004). The moment formula for implied volatility at extreme strikes. Mathematical Finance 14(3), 469-480.

Leippold, M. and L. Wu (2002). Asset pricing under the quadratic class. Journal of Financial and Quantitative Analysis 37(2). 
Lewis, A. (2000). Option Valuation under Stochastic Volatility. Finance Press.

Lewis, A. (2001). A simple option formula for general jump-diffusion and other exponential lévy processes. OptionCity.net Publications.

Lian, G.-H. and S.-P. Zhu (2011). Pricing vix options with stochastic volatility and random jumps. Decisions in Economics and Finance, 1-18.

Lipton, A. (2002). The vol smile problem. RISK 15(2), 61-66.

Longstaff, F. and E. Schwartz (2001). Valuing american options by simulation: A simple least-squares approach. Review of FinancialSstudies 14(1), 113-147.

Madan, D., P. Carr, and E. Chang (1998). The variance gamma process and option pricing. European Finance Review 2(1), 79-105.

Madan, D. and E. Seneta (1990). The variance gamma model for share market returns. Journal of Business, 511-524.

Medvedev, A. and O. Scaillet (2007). Approximation and calibration of short-term implied volatilities under jump-diffusion stochastic volatility. Review of Financial Studies 20(2), 427-459.

Morini, M. and A. Prampolini (2011). Risky funding: A unified framework for counterparty and liquidity charges. Risk Magazine March.

Nadarajah, S. and S. Kotz (2007). On the Weibull MGF. IEEE Transactions on Communications 55(7), 1287-1287.

Nicolato, E. and E. Venardos (2003). Option pricing in stochastic volatility models of the ornstein-uhlenbeck type. Mathematical Finance 13(4), 445-466.

Nicolato, N. and D. Sloth (2013). Approximation of option prices under time-changed dynamics. Quantitative Finance (Forthcoming).

Osajima, Y. (2007). Asymptotic expansion formula of implied volatility for dynamic sabr model and fx hybrid model. Unpublished Manuscript.

Pallavicini, A., D. Perini, and D. Brigo (2011). Funding valuation adjustment: a consistent framework including cva, dva, collateral, netting rules and re-hypothecation. arXiv preprint. 
Pallavicini, A., D. Perini, and D. Brigo (2012). Funding, collateral and hedging: uncovering the mechanics and the subtleties of funding valuation adjustments. arXiv preprint arXiv:1210.3811.

Parker, E. and A. McGarry (2009). The isda master agreement and csa: Close-out weaknesses exposed in the banking crisis and suggestions for change. Butterworths Journal of International Banking Law 1.

Piterbarg, V. (2010). Funding beyond discounting: collateral agreements and derivatives pricing. Risk Magazine 2, 97-102.

Poulsen, R. and A. Ribeiro (2012). Approximation behooves calibration. Working Paper, University of Copenhagen.

Renault, E. and N. Touzi (1996). Option hedging and implied volatilities in a stochastic volatility model. Mathematical Finance 6(3), 279-302.

Revuz, D. and M. Yor (1999). Continuous Martingales And Brownian Motion. Springer.

Rogers, L. and O. Zane (1999). Saddlepoint approximations to option prices. The Annals of Applied Probability 9(2), 493-503.

Romano, M. and N. Touzi (1997). Contingent claims and market completeness in a stochastic volatility model. Mathematical Finance 7(4), 399-412.

Sartorelli, G. (2010). Edgeworth expansion for the computation of plain vanilla prices in the heston model. Unpublished Manuscript.

Sato, K. (1999). Lévy Processes and Infinitely Divisible Distributions. Cambridge University Press.

Schmelzle, M. (2010). Option pricing formulae using fourier transform: Theory and application. Unpublished Manuscript.

Schoutens, W. (2002). The meixner process: Theory and applications in finance.

Schoutens, W., E. Simons, and J. Tistaert (2004). A perfect calibration! Now what. Wilmott Magazine 2, 66-78. 
Sepp, A. (2003). Fourier transform for option pricing under affine jump-diffusions: An overview. Unpublished Manuscript.

Sepp, A. (2008a). Pricing options on realized variance in the heston model with jumps in returns and volatility. Journal of Computational Finance 11(4), 33-70.

Sepp, A. (2008b). Vix option pricing in a jump-diffusion model. Risk Magazine, 84-89.

Soulier, P. (2009). Some applications of regular variation in probability and statistics.

Stehfest, H. (1970). Algorithm 368: Numerical inversion of laplace transforms. Communications of the ACM 13(1), 47-49.

Stein, E. M. and J. C. Stein (1991). Stock price distributions with stochastic volatility: an analytic approach. Review of financial Studies 4(4), 727-752.

Tankov, P. (2011). Pricing and hedging in exponential Lévy models:Review of recent results, Volume 2003 of Lecture Notes on Mathematical Finance, pp. 319-359. Springer.

Tehranchi, M. (2009). Asymptotics of implied volatility far from maturity. Journal of Applied Probability 46(3), 629-650.

Tilley, J. (1993). Valuing american options in a path simulation model. Transactions of the Society of Actuaries 45(83), 104.

Tsitsiklis, J. and B. Van Roy (2001). Regression methods for pricing complex americanstyle options. IEEE Transactions on Neural Networks 12(4), 694-703.

Voss, J. (2009). Upper and lower bounds in exponential tauberian theorems. Unpublished Manuscript.

Weeber, P. and E. Robson (2009). Market practices for settling derivatives in bankruptcy. ABI Journal 34-35 (9), 76-78.

Weeks, W. (1966). Numerical inversion of laplace transforms using laguerre functions. Journal of the ACM 13(3), 419-429.

Xiong, J., A. Wong, and D. Salopek (2005). Saddlepoint approximations to option price in a general equilibrium model. Statistics \& Probability Letters 71(4), 361-369. 
Yu, J. (2007). Closed-form likelihood approximation and estimation of jump-diffusions with an application to the realignment risk of the chinese yuan. Journal of Econometrics $141(2), 1245-1280$. 


\section{DEPARTMENT OF ECONOMICS AND BUSINESS \\ AARHUS UNIVERSITY \\ SCHOOL OF BUSINESS AND SOCIAL SCIENCES \\ www.econ.au.dk}

\section{PhD Theses since 1 July 2011}

2011-4 Anders Bredahl Kock: Forecasting and Oracle Efficient Econometrics

2011-5 Christian Bach: The Game of Risk

2011-6 Stefan Holst Bache: Quantile Regression: Three Econometric Studies

2011:12 Bisheng Du: Essays on Advance Demand Information, Prioritization and Real Options in Inventory Management

2011:13 Christian Gormsen Schmidt: Exploring the Barriers to Globalization

2011:16 Dewi Fitriasari: Analyses of Social and Environmental Reporting as a Practice of Accountability to Stakeholders

2011:22 Sanne Hiller: Essays on International Trade and Migration: Firm Behavior, Networks and Barriers to Trade

2012-1 Johannes Tang Kristensen: From Determinants of Low Birthweight to Factor-Based Macroeconomic Forecasting

2012-2 Karina Hjortshøj Kjeldsen: Routing and Scheduling in Liner Shipping

2012-3 Soheil Abginehchi: Essays on Inventory Control in Presence of Multiple Sourcing

2012-4 Zhenjiang Qin: Essays on Heterogeneous Beliefs, Public Information, and Asset Pricing

2012-5 Lasse Frisgaard Gunnersen: Income Redistribution Policies

2012-6 Miriam Wüst: Essays on early investments in child health

2012-7 Yukai Yang: Modelling Nonlinear Vector Economic Time Series

2012-8 Lene Kjærsgaard: Empirical Essays of Active Labor Market Policy on Employment

2012-9 Henrik Nørholm: Structured Retail Products and Return Predictability

2012-10 Signe Frederiksen: Empirical Essays on Placements in Outside Home Care 
2012-11 Mateusz P. Dziubinski: Essays on Financial Econometrics and Derivatives Pricing

2012-12 Jens Riis Andersen: Option Games under Incomplete Information

2012-13 Margit Malmmose: The Role of Management Accounting in New Public Management Reforms: Implications in a Socio-Political Health Care Context

2012-14 Laurent Callot: Large Panels and High-dimensional VAR

2012-15 Christian Rix-Nielsen: Strategic Investment

2013-1 Kenneth Lykke Sørensen: Essays on Wage Determination

2013-2 Tue Rauff Lind Christensen: Network Design Problems with Piecewise Linear Cost Functions

2013-3 Dominyka Sakalauskaite: A Challenge for Experts: Auditors, Forensic Specialists and the Detection of Fraud

2013-4 Rune Bysted: Essays on Innovative Work Behavior

2013-5 Mikkel Nørlem Hermansen: Longer Human Lifespan and the Retirement Decision

2013-6 Jannie H.G. Kristoffersen: Empirical Essays on Economics of Education

2013-7 Mark Strøm Kristoffersen: Essays on Economic Policies over the Business Cycle

2013-8 Philipp Meinen: Essays on Firms in International Trade

2013-9 Cédric Gorinas: Essays on Marginalization and Integration of Immigrants and Young Criminals - A Labour Economics Perspective

2013-10 Ina Charlotte Jäkel: Product Quality, Trade Policy, and Voter Preferences: Essays on International Trade

2013-11 Anna Gerstrøm: World Disruption - How Bankers Reconstruct the Financial Crisis: Essays on Interpretation

2013-12 Paola Andrea Barrientos Quiroga: Essays on Development Economics

2013-13 Peter Bodnar: Essays on Warehouse Operations

2013-14 Rune Vammen Lesner: Essays on Determinants of Inequality

2013-15 Peter Arendorf Bache: Firms and International Trade

2013-16 Anders Laugesen: On Complementarities, Heterogeneous Firms, and International Trade 
2013-17 Anders Bruun Jonassen: Regression Discontinuity Analyses of the Disincentive Effects of Increasing Social Assistance

2014-1 David Sloth Pedersen: A Journey into the Dark Arts of Quantitative Finance 
